\title{
On the 2D Ericksen-Leslie equations with anisotropic energy and external forces
}

\author{
Zdzislaw BrzeźniaK(iD, Gabriel Deugoué and Paul André RaZaFimandimby
}

Abstract. In this paper we consider the 2D Ericksen-Leslie equations which describe the hydrodynamics of nematic liquid crystal with external body forces and anisotropic energy modeling the energy of applied external control such as magnetic or electric field. Under general assumptions on the initial data, the external data and the anisotropic energy, we prove the existence and uniqueness of global weak solutions with finitely many singular times. If the initial data and the external forces are sufficiently small, then we establish that the global weak solution does not have any singular times and is regular as long as the data are regular.

\section{Contents}

1. Introduction

2. Notation and preliminaries

2.1. Notations for the velocity field $v$

2.2. The Laplacian for the director field $d$

2.3. An abstract formulation of problem (1.1)

3. The existence and the uniqueness of a regular solution to problem (2.13)

4. The existence and the uniqueness of a maximal local regular solution to $(2.13)$

5. The existence and the uniqueness of a global weak solution

6. On the regularity and the set of singular times of the solutions when the data are small

Mathematics Subject Classification: 35K45, 35K55

Keywords: Liquid Crystal, Ericksen-Leslie equations, Magnetic Field, Electric Field, External Forcing, Anisotropic Potential.

This article is part of a project that is currently funded by the European Union's Horizon 2020 research and innovation programme under the Marie Skłodowska-Curie Grant Agreement No. 791735 "SELEs".

P. Razafimandimby is very grateful to the warm hospitality of the Lehrstuhl für Angewandte Mathematik, Montanuniversität Leoben, Leoben (Austria), where part of the paper was written during the period 16 December 2019 till 30 January 2020.

G. Deugoue is thankful to the financial support and the hospitality of the Department of Mathematics of the University of York during his visit in June 2019. He is also very grateful to the financial support from the Marie Skłodowska-Curie Grant (Agreement No. 791735) "SELEs". 
Appendix B: Weak solution of a modified viscous transport equation 3953 Appendix C: Proof of Claim 4.10

\section{Introduction}

We consider a hydrodynamical system modeling the flow of liquid crystal materials with anisotropic energy in a $2 \mathrm{D}$ bounded domain. More precisely, let $T>0$ and $\Omega \subset \mathbb{R}^{2}$ be a bounded domain with a smooth boundary $\partial \Omega$ and let us consider

$$
\begin{aligned}
& \partial_{t} v+v \cdot \nabla v-\Delta v+\nabla \mathrm{p}=-\operatorname{div}(\nabla d \odot \nabla d)+f, \text { in }[0, T) \times \Omega \\
& \partial_{t} d+v \cdot \nabla d=-d \times\left(d \times\left(\Delta d-\phi^{\prime}(d)\right)\right)+d \times g, \text { in }[0, T) \times \Omega, \\
& \operatorname{div} v=0, \text { in }[0, T) \times \Omega, \\
& v=\frac{\partial d}{\partial v}=0, \text { on }[0, T) \times \partial \Omega, \\
& |d|=1, \text { in }[0, T) \times \Omega, \\
& (v(0), d(0))=\left(v_{0}, d_{0}\right), \text { in } \Omega,
\end{aligned}
$$

where $v:[0, T) \times \Omega \rightarrow \mathbb{R}^{2}, d:[0, T) \times \Omega \rightarrow \mathbb{S}^{2}$, where $\mathbb{S}^{2}$ is the unit sphere in $\mathbb{R}^{3}$, and $p:[0, T) \times \Omega \rightarrow \mathbb{R}$ represent the velocity field of the flow, the macroscopic molecular orientation of the liquid crystal material and the pressure function, respectively. In the system (1.1), the function $\phi: \mathbb{R}^{3} \rightarrow \mathbb{R}^{+}$is a given map, $f:[0, T) \times \Omega \rightarrow \mathbb{R}^{2}$ and $g:[0, T) \times \Omega \rightarrow \mathbb{R}^{3}$ are given external forces. The symbol $v(x)$ is a unit outward normal vector at each point $x \in \partial \Omega$. The matrix $\nabla d \odot \nabla d$ is defined by

$$
[\nabla d \odot \nabla d]_{i j}=\sum_{k=1}^{3} \partial_{i} d_{k} \partial_{j} d_{k} \text { for } 1 \leq i, j \leq 3 .
$$

Using the identities

$$
\begin{aligned}
& -\operatorname{div}(\nabla d \odot \nabla d)=-\nabla d \Delta d+\frac{1}{2} \nabla|\nabla d|^{2}, \\
& a \times(b \times c)=(a \cdot c) b-(a \cdot b) c \text { for } a, b, c \in \mathbb{R}^{3},
\end{aligned}
$$

we can rewrite system (1.1) as follows:

$$
\begin{aligned}
& \partial_{t} v+v \cdot \nabla v-\Delta v+\nabla \tilde{\mathrm{p}}=-\nabla d \Delta d+f, \\
& \partial_{t} d+v \cdot \nabla d=\Delta d+|\nabla d|^{2} d-\phi^{\prime}(d)+\left(\phi^{\prime}(d) \cdot d\right) d+d \times g, \\
& \operatorname{div} v=0, \\
& v_{\mid \partial \Omega}=\left.\frac{\partial d}{\partial v}\right|_{\partial \Omega}=0,
\end{aligned}
$$




$$
\begin{aligned}
& |d|=1, \\
& (v(0), d(0))=\left(v_{0}, d_{0}\right),
\end{aligned}
$$

where

$$
\tilde{\mathrm{p}}=\mathrm{p}+\frac{1}{2}|\nabla d|^{2} .
$$

While we focus our mathematical analysis on the system (1.2) with the Dirichlet and the Neumann boundary conditions (1.2d), our results remain valid for the case of the periodic boundary conditions. That is, our results remain true in the case that $\Omega$ is a $2 \mathrm{D}$ torus $\mathbb{T}^{2}$ and $(1.2 \mathrm{~d})$ is replaced by

$$
\int_{\Omega} v(t, x) \mathrm{d} x=0, \forall t \in(0, T] .
$$

The model (1.2) is an oversimplification of a Ericksen-Leslie model of nematic liquid crystal with a simplified energy density

$$
\frac{1}{2}|\nabla d|^{2}+\phi(d) .
$$

The term $\frac{1}{2}|\nabla d|^{2}$ represents the one-constant simplification of the Frank-Oseen energy density and $\phi(d)$ represents an anisotropic energy density. One example of such anisotropic energy density is the magnetic energy density

$$
\phi(n)=(n \cdot H)^{2},
$$

when the nematic liquid crystal is subjected to the action of a constant magnetic field $H \in \mathbb{R}^{3}$. We also give different examples of mathematical models of anisotropic energy density later on. For more details on physical modeling of liquid crystal under the action of external control such as magnetic or electric field we refer to the books [9] and [34] and the papers [10] and [23].

We should note that since (1.2) was obtained by neglecting several terms such as the viscous Leslie stress tensor in the equation for $v$ (see, for instance, [27,28]), the stretching and rotational effects for $d$, one does not known whether it is thermodynamically stable or consistent with the laws of thermodynamics. However, this model still retains many mathematical and essential features of the hydrodynamic equations for nematic liquid crystals. In recent years, several liquid crystal models which are thermodynamically consistent and stable have been recently developed and analyzed, see, for instance, the [11-16,29,31] and references therein.

In the absence of external forcings $f, g$ and the anisotropic energy potential $\phi(d)$, the system (1.2) has extensively studied and several important results have been obtained. In addition to the papers we cited above we refer, among others, to $[17,18,26-28,30$, 41] for results obtained prior to 2013, and to [7,19-22,24,25,40,42,43] for results obtained after 2014. For detailed reviews of the literature about the mathematical 
theory of nematic liquid crystals and other related models, we recommend the review articles [8,15,29] and the recent papers [20,25].

Let us now outline the contributions of our manuscript.

(C1) In Sect. 3, we prove by using Banach fixed point theorem that if $\left(v_{0}, d_{0}\right) \in$ $D\left(\mathrm{~A}^{\frac{1}{2}}\right) \times(D(\hat{\mathrm{A}}) \cap \mathcal{M})$ and $(f, g) \in L^{2}(0, T ; \mathrm{H} \times D(\hat{\mathrm{A}}))$, then there exists a unique local regular solution $(v, d):\left[0, T_{0}\right] \rightarrow D\left(\mathrm{~A}^{\frac{1}{2}}\right) \times D(\hat{\mathrm{A}}) \cap \mathcal{M}$ of problem (1.2) such that $(v, d) \in C\left(\left[0, T_{0}\right] ; D\left(\mathrm{~A}^{\frac{1}{2}}\right) \times(D(\hat{\mathrm{A}}) \cap \mathcal{M})\right) \cap L^{2}\left(0, T_{0} ; D(\mathrm{~A}) \times D\left(\hat{\mathrm{A}}^{\frac{3}{2}}\right)\right.$, see Theorem 3.7. Here,

$$
\mathcal{M}=\left\{d: \Omega \rightarrow \mathbb{R}^{3}:|d(x)|=1 \text { Leb-a.e. }\right\},
$$

A and $\hat{A}$ are, respectively, the Stokes operator and the Neumann Laplacian, see Sect. 2 for the definitions of these operators and the space $\mathrm{H}$.

(C2) We exploit this result and the local energy method developed in [26,36] and [17] to show in Sect. 4 that there exists universal constants $\varepsilon_{0}>0$ and $r_{0}>0$ such that the following statement holds.

If $(f, g) \in L^{2}\left(0, T ; \mathrm{H} \times D\left(\hat{\mathrm{A}}^{1 / 2}\right)\right)$ and $\left(v_{0}, d_{0}\right)$ belongs to $\mathrm{V} \times(D(\hat{\mathrm{A}}) \cap \mathcal{M})$ with small energy, i.e., there exists $R_{0} \in\left(0, r_{0}\right]$ such that

$$
\sup _{x \in \Omega} \int_{B\left(x, R_{0}\right)}\left[\left|v_{0}(x)\right|^{2}+\left|\nabla d_{0}(x)\right|^{2}+\phi\left(d_{0}(x)\right)\right] \mathrm{d} x<\varepsilon_{0}^{2},
$$

then, there exists a unique maximal local regular solution $(v, d):\left[0, T_{0}\right] \rightarrow D\left(\mathrm{~A}^{\frac{1}{2}}\right) \times$ $(D(\hat{\mathrm{A}}) \cap \mathcal{M})$ of problem $(1.2)$ such that $(v, d) \in C\left(\left[0, T_{0}\right] ; D\left(\mathrm{~A}^{\frac{1}{2}}\right) \times(D(\hat{\mathrm{A}}) \cap \mathcal{M})\right) \cap$ $L^{2}\left(0, T_{0} ; D(\mathrm{~A}) \times D\left(\hat{\mathrm{A}}^{\frac{3}{2}}\right)\right.$ and

$$
\frac{1}{2} \sup _{0 \leq t \leq T_{0}} \sup _{x \in \Omega} \int_{B\left(x, R_{0}\right)}\left(|v(t, y)|^{2}+|\nabla d(t, y)|^{2}+2 \phi(d(t, y))\right) \mathrm{d} y \leq 2 \varepsilon_{1}^{2} .
$$

We refer to Proposition 4.3 and its proof for more details.

(C3) The two results above are exploited in Sect. 5 in order to prove the existence and the uniqueness of a global weak existence $(\mathbf{v}, \mathbf{d})$ to problem (1.2). The solution $(\mathbf{v}, \mathbf{d}):[0, T] \rightarrow \mathrm{H} \times \mathrm{H}^{1}$ is proven to have a finite number of time singularities $T_{1}, \ldots, T_{L}$ when the target space $\mathrm{H} \times \mathrm{H}^{1}$ are equipped with strong topology. However, $(\mathbf{v}, \mathbf{d}):[0, T] \rightarrow \mathrm{H} \times \mathrm{H}^{1}$ is weakly continuous, which enables us to define $(\mathbf{v}, \mathbf{d})$ at each time singularity $T_{i}, i=1, \ldots, L$. Our main result is presented in Theorem 5.6 and can be summarized as follows.

Theorem 1.1. Let $\left(v_{0}, d_{0}\right) \in \mathrm{H} \times\left(\mathrm{H}^{1} \cap \mathcal{M}\right)$. Then, there exist constants $\varrho_{0}>0$ and $\varepsilon_{0}>0$ such that the following hold. If $(f, g) \in L^{2}\left(0, T ; \mathrm{H}^{-1} \times L^{2}\right)$, then there exist

(i) a number $L \in \mathbb{N}$, depending only on the norms of $\left(v_{0}, d_{0}\right) \in \mathrm{H} \times \mathrm{H}^{1}$ and $(f, g) \in L^{2}\left(0, T ; \mathrm{H}^{-1} \times L^{2}\right)$, a finite sequence $0=T_{0}<T_{1}<\cdots<T_{L} \leq T$ and,

(ii) a global weak solution $(\mathbf{v}, \mathbf{d})$ of problem $(1.2)$ such that $(\mathbf{v}, \mathbf{d}) \in C_{w}([0, T] ; \mathrm{H} \times$ $\left.\mathrm{H}^{1}\right) \cap L^{2}(0, T ; \mathrm{V} \times D(\hat{\mathrm{A}}))$ and for all $t \in[0, T], \mathbf{d}(t) \in \mathcal{M}$ with 


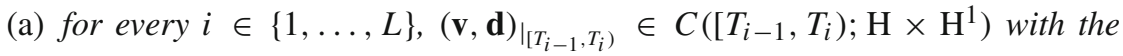
left-limit at $T_{i}$, which satisfies the variational form of problem (1.2) on the interval $\left[T_{i-1}, T_{i}\right)$ with initial data $\left(v\left(T_{i-1}\right), d\left(T_{i-1}\right)\right)$.

(b) If $T_{L}<T$, then $(\mathbf{v}, \mathbf{d})_{\left.\right|_{\left[T_{L}, T\right]}}$ belongs to $C\left(\left[T_{L}, T\right] ; \mathrm{H} \times \mathrm{H}^{1}\right)$ and satisfies the variational form problem (1.2) on the interval $\left[T_{L}, T\right]$ with initial data $\left(\mathbf{v}\left(T_{L}\right), \mathbf{d}\left(T_{L}\right)\right)$.

(c) There exists $\varepsilon_{1} \in\left(0, \varepsilon_{0}\right)$ such that for all $i \in\{1, \ldots, L\}$ and all $R \in\left(0, \varrho_{0}\right]$

$$
\lim _{t \nearrow T_{i}} \sup _{x \in \Omega} \int_{B(x, R)}\left(\frac{1}{2}|\mathbf{v}(t, y)|^{2}+\frac{1}{2}|\nabla \mathbf{d}(t, y)|^{2}+\phi(d(t, y))\right) \mathrm{d} y \geq \varepsilon_{1}^{2} .
$$

(d) At every time $T_{i}, i \in\{1, \ldots, L\}$, there is a loss of energy at least $\varepsilon_{1}^{2} \in\left(0, \varepsilon_{0}^{2}\right)$, i.e.,

$$
\begin{aligned}
& \int_{\Omega}\left(\frac{1}{2}\left|\mathbf{v}\left(T_{i}, y\right)\right|^{2}+\frac{1}{2}\left|\nabla \mathbf{d}\left(T_{i}, y\right)\right|^{2}+\phi\left(d\left(T_{i}, y\right)\right)\right) \mathrm{d} y \\
& \leq \int_{\Omega}\left(\frac{1}{2}\left|\mathbf{v}\left(T_{i-1}, y\right)\right|^{2}+\frac{1}{2}\left|\nabla \mathbf{d}\left(T_{i-1}, y\right)\right|^{2}+\phi\left(d\left(T_{i-1}, y\right)\right)\right) \mathrm{d} y \\
& \quad+\frac{1}{2} \int_{T_{i-1}}^{T_{1}}\left[|f|_{\mathrm{H}^{-1}}^{2}+|g|_{L^{2}}^{2}\right] \mathrm{d} t-\varepsilon_{1}^{2} .
\end{aligned}
$$

The numbers $T_{1}, \cdots, T_{L}$ are called the singular times of the solution $(\mathbf{v}, \mathbf{d})$.

Because of the presence of the anisotropic energy and the external forces, this result is a generalization of the global existence of weak solution obtained in [17] and [26]. (C4) We also prove that the weak solution $(\mathbf{v}, \mathbf{d}) \in C_{w}\left([0, T] ; \mathrm{H} \times \mathrm{H}^{1}\right) \cap L^{2}(0, T ; \mathrm{V} \times$ $D(\hat{\mathrm{A}}))$ is unique, see Proposition 5.5 and Remark 5.7.

(C5) Finally, in Sect. 6 we prove that the set of singular times is empty when the data $\left(v_{0}, d_{0}\right) \in \mathrm{H} \times \mathrm{H}^{1}$ and $(f, g) \in L^{2}\left(0, T ; \mathrm{H}^{-1} \times L^{2}\right)$ are sufficiently small. We also show that if the data are sufficiently regular and small, i.e., $\left(v_{0}, d_{0}\right) \in D\left(\mathrm{~A}^{\frac{1}{2}}\right) \times D(\hat{\mathrm{A}})$ and $(f, g) \in L^{2}\left(0, T ; \mathrm{H} \times \mathrm{H}^{1}\right)$, then the weak solution becomes regular for all time. Moreover, for all $t \in[0, T)(v(t), d(t))$ lies in a compact set of $\mathrm{H} \times \mathrm{H}^{1}$. We refer the reader to Theorems 6.3 and 6.5 for more precision and details.

We close this introduction with the presentation of the layout of the present paper. In Sect. 2 we fix the frequently used notation in the manuscript. We also state and prove some auxiliary results which are essential to our analysis. Section 3 is devoted to the existence and uniqueness of a regular solution to Problem (2.13). In Sect. 4 we prove that one can find a small number $R_{0}>0$ and a unique maximal local regular solution $\left((v, d) ; T_{0}\right)$ such that its energy at any time $t \in\left[0, T_{0}\right)$ does not exceed twice the supremum of all energies on $B\left(x, 2 R_{0}\right), x \in \Omega$ of the initial data. This result will play a pivotal role in the proof of the existence of a maximal local strong solution to Problem (2.13) in Sect. 5. 


\section{Notation and preliminaries}

Let $\Omega \subset \mathbb{R}^{2}$ be an open and bounded set. We denote by $\Gamma=\partial \Omega$ the boundary of $\Omega$. We assume that the closure $\bar{\Omega}$ of the set $\Omega$ is a manifold with $C^{\infty}$ boundary $\Gamma:=\partial \Omega$ which is a 1-dimensional infinitely differentiable manifold being locally on one side of $\Omega$.

Throughout this paper $L^{p}\left(\Omega ; \mathbb{R}^{\ell}\right), \mathrm{W}^{p, k}\left(\Omega ; \mathbb{R}^{\ell}\right)\left(\mathrm{H}^{k}\left(\Omega ; \mathbb{R}^{\ell}\right)=\mathrm{W}^{2, p}\left(\Omega ; \mathbb{R}^{\ell}\right)\right)$, $p \in[1, \infty], k \in \mathbb{N}$, and $\mathrm{H}^{\alpha}\left(\Omega ; \mathbb{R}^{\ell}\right), \alpha \in(0, \infty)$, denote the Lebesgue and Sobolev spaces whose elements take values in $\mathbb{R}^{\ell}, \ell=2,3$. To shorten the notation we will just write $L^{p}, \mathrm{~W}^{p, k}, \mathrm{H}^{k}$ and $\mathrm{H}^{\alpha}$ irrespectively if the elements of these spaces take values in $\mathbb{R}^{2}$ or $\mathbb{R}^{3}$.

\subsection{Notations for the velocity field $v$}

The following is an abridged version of notations and preliminary of the paper [2]. The facts we enumerate here can be found in [2, Section 2] and references therein. Let $\mathcal{D}(\Omega)$ (resp. $\mathcal{D}(\bar{\Omega})$ ) be the set of all $C^{\infty}$ class vector fields $u: \mathbb{R}^{2} \rightarrow \mathbb{R}^{2}$ with compact support contained in the set $\Omega$ (resp. $\bar{\Omega}$ ). Then, let us define

$$
\begin{aligned}
E(\Omega) & =\left\{u \in L^{2}\left(\Omega, \mathbb{R}^{2}\right): \operatorname{div} u \in L^{2}\left(\Omega, \mathbb{R}^{2}\right)\right\}, \\
\mathcal{V} & =\left\{u \in C_{0}\left(\Omega, \mathbb{R}^{2}\right): \operatorname{div} u=0\right\}, \\
\mathrm{H} & =\text { the closure of } \mathcal{V} \text { in } L^{2}\left(\Omega, \mathbb{R}^{2}\right), \\
\mathrm{H}_{0}^{1}\left(\Omega, \mathbb{R}^{2}\right) & =\text { the closure of } \mathcal{D}\left(\Omega, \mathbb{R}^{2}\right) \text { in } \mathrm{H}^{1}\left(\Omega, \mathbb{R}^{2}\right), \\
\mathrm{V} & =\text { the closure of } \mathcal{V} \text { in } \mathrm{H}_{0}^{1}\left(\Omega, \mathbb{R}^{2}\right) .
\end{aligned}
$$

The inner products in all $L^{2}$ spaces will be denoted by $\langle\cdot, \cdot\rangle$. The space $E(\Omega)$ is a Hilbert space with a scalar product

$$
\langle u, v\rangle_{E(\Omega)}:=\langle u, v\rangle+\langle\operatorname{div} u, \operatorname{div} v\rangle .
$$

We endow the set $\mathrm{H}$ with the inner product $\rangle \cdot, \cdot\rangle$ and the norm $|\cdot|_{\mathrm{H}}$ induced by $L^{2}$.

The space $\mathrm{H}$ can also be characterized in the following way, see

[37, Theorem I.1.4],

$$
\mathrm{H}=\left\{u \in E(\Omega): \operatorname{div} u=0 \text { and } u \cdot v_{\left.\right|_{\partial \Omega}}=0\right\} .
$$

Let us denote by $\Pi: L^{2}\left(\Omega, \mathbb{R}^{2}\right) \rightarrow \mathrm{H}$ the orthogonal projection called usually the Leray-Helmholtz projection. It is known, see [37, Remark I.1.6] that $\Pi$ maps continuously the Sobolev space $\mathrm{H}^{1}$ into itself.

Observe that $\Omega$ is a Poincaré domain, i.e., there exists a constant $\lambda_{1}>0$ such that the following Poincaré inequality is satisfied

$$
\lambda_{1} \int_{\Omega} \varphi^{2}(x) \mathrm{d} x \leq \int_{\Omega}|\nabla \varphi(x)|^{2} \mathrm{~d} x, \quad \varphi \in \mathrm{H}_{0}^{1}(\Omega) .
$$


Because of this the norms on the space $\mathrm{V}$ induced by $|\cdot|_{\mathrm{H}}^{1}=|\cdot|_{L^{2}}+|\nabla \cdot|_{L^{2}}$ and by $|\nabla \cdot|_{L^{2}}$ are equivalent. Since the space $\mathrm{V}$ is densely and continuously embedded into $\mathrm{H}$, by identifying $\mathrm{H}$ with its dual $\mathrm{H}^{\prime}$, we have the following embedding

$$
\mathrm{V} \hookrightarrow \mathrm{H} \cong \mathrm{H}^{\prime} \hookrightarrow \mathrm{V}^{\prime} .
$$

Let us observe here that, in particular, the spaces $\mathrm{V}, \mathrm{H}$ and $\mathrm{V}^{\prime}$ form a Gelfand triple.

We will denote by $|\cdot| \mathrm{V}^{*}$ and $\langle\cdot, \cdot\rangle$ the norm in $\mathrm{V}^{*}$ and the duality pairing between $\mathrm{V}$ and $\mathrm{V}^{*}$, respectively.

Now, define the bilinear form $a: \mathrm{V} \times \mathrm{V} \rightarrow \mathbb{R}$ by setting

$$
a(u, v):=\langle\nabla u, \nabla v\rangle, \quad u, v \in \mathrm{V}
$$

It is well known that this bilinear map is $\mathrm{V}$-continuous and $\mathrm{V}$-coercive, i.e., there exist some $C_{0}, C_{1}>0$ such that

$$
C_{0}|u|_{\mathrm{V}}^{2} \leq|a(u, u)| \leq C|u|_{\mathrm{V}}^{2}, \quad u \in \mathrm{V}
$$

Hence, by the Riesz lemma and Lax-Milgram theorem, see, for instance, Temam [37, Theorem II.2.1], there exists a unique isomorphism $\mathcal{A}: \mathrm{V} \rightarrow \mathrm{V}^{\prime}$, such that $a(u, v)=\langle\mathcal{A} u, v\rangle$, for $u, v \in \mathrm{V}$.

Next we define an unbounded linear operator $\mathrm{A}$ in $\mathrm{H}$ as follows:

$$
\left\{\begin{array}{l}
D(\mathrm{~A})=\{u \in \mathrm{V}: \mathcal{A} u \in \mathrm{H}\}, \\
\mathrm{A} u=\mathcal{A} u, u \in D(\mathrm{~A}) .
\end{array}\right.
$$

Under our assumption on $\Omega$, A and $D(\mathrm{~A})$ can be characterized as follows see for example [39],

$$
\left\{\begin{array}{l}
D(\mathrm{~A})=\mathrm{V} \cap \mathrm{H}^{2}=\mathrm{H} \cap \mathrm{H}_{0}^{1} \cap \mathrm{H}^{2}, \\
\mathrm{~A} u=-\mathrm{P} \Delta u, \quad u \in D(\mathrm{~A}) .
\end{array}\right.
$$

It is also well known, see [2, Section 2] and references therein, that A is a positive self-adjoint operator in $\mathrm{H}$ and

$$
D\left(\mathrm{~A}^{\alpha / 2}\right)=[\mathrm{H}, D(\mathrm{~A})]_{\frac{\alpha}{2}},
$$

where $[\cdot, \cdot]_{\frac{\alpha}{2}}$ is the complex interpolation functor of order $\frac{\alpha}{2}$. Furthermore, for $\alpha \in$ $\left(0, \frac{1}{2}\right)$

$$
D\left(\mathrm{~A}^{\alpha / 2}\right)=\mathrm{H} \cap \mathrm{H}^{\alpha}\left(\Omega, \mathbb{R}^{2}\right) .
$$

In particular, $\mathrm{V}=D\left(\mathrm{~A}^{1 / 2}\right)$ and $\left|\mathrm{A}^{\frac{1}{2}} u\right|^{2}=|\nabla u|^{2}=:\|u\|^{2}$ for $u \in \mathrm{V}$. The equality (2.7) leads to the following result which was proved in [2, Proposition 2.1].

Proposition 2.1. Assume that $\alpha \in\left(0, \frac{1}{2}\right)$. Then the Leray-Helmholtz projection $\Pi i s$ a well-defined and continuous map from $\mathrm{H}^{\alpha}$ into $D\left(\mathrm{~A}^{\alpha / 2}\right)$. 
Let us finally recall that the projection $\Pi$ extends to a bounded linear projection in the space $L^{q}$, for any $q \in(1, \infty)$.

Now, consider the trilinear form $b$ on $V \times V \times V$ given by

$$
b(u, v, w)=\sum_{i, j=1}^{2} \int_{\Omega} u_{i} \frac{\partial v_{j}}{\partial x_{i}} w_{j} d x, \quad u, v, w \in \mathrm{V} .
$$

Indeed, $b$ is a continuous trilinear form such that

$$
b(u, v, w)=-b(u, w, v), \quad u \in \mathrm{V}, v, w \in \mathrm{H}_{0}^{1}\left(\Omega, \mathbb{R}^{2}\right),
$$

or a proof see, for instance, [37, Lemma 1.3, p.163] .

Define next the bilinear map $B: \mathrm{V} \times \mathrm{V} \rightarrow \mathrm{V}^{*}$ by setting

$$
\mathrm{V}^{*}\langle B(u, v), w\rangle_{\mathrm{V}}=b(u, v, w), \quad u, v, w \in \mathrm{V},
$$

and the homogenous polynomial of second degree $B: \mathrm{V} \rightarrow \mathrm{V}^{*}$ by

$$
B(u)=B(u, u), u \in \mathrm{V} .
$$

Let us observe that if $v \in D(\mathrm{~A})$, then $B(u, v) \in H$ and the following identity is a direct consequence of (2.8).

$$
\mathrm{V}^{*}\langle\mathrm{~B}(u, v), v\rangle_{\mathrm{V}}=0, \quad u, v \in \mathrm{V} .
$$

The restriction of the map $\mathrm{B}$ to the space $D(\mathrm{~A}) \times D(\mathrm{~A})$ has also the following representation

$$
\mathrm{B}(u, v)=\Pi(u \cdot \nabla v), \quad u, v \in D(\mathrm{~A}),
$$

where $\Pi$ is the Leray-Helmholtz projection operator and $u \nabla v=\sum_{j=1}^{2} u^{j} D_{j} v \in$ $L^{2}\left(\Omega, \mathbb{R}^{2}\right)$.

\subsection{The Laplacian for the director field $d$}

Throughout this section, we still denote $L^{2}\left(\Omega ; \mathbb{R}^{3}\right)$ and $\mathrm{H}^{k}\left(\Omega ; \mathbb{R}^{3}\right), k \in \mathbb{N}$, by $L^{2}$ and $H^{1}$, respectively. We aim in this subsection to introduce the Laplacian for the director $d: \Omega \rightarrow \mathbb{R}^{3}$ with the Neumann boundary conditions. We can do this by mimicking the way we define the Stokes operator A. We define the bilinear map $\hat{a}: \mathrm{H}^{1} \times \mathrm{H}^{1} \rightarrow \mathbb{R}$ by

$$
\hat{a}(d, n)=\int_{\Omega}(\nabla d \nabla n) \mathrm{d} x=\sum_{i=1}^{3} \sum_{j=1}^{3} \int_{\Omega}\left(\partial_{i} d_{j} \partial_{i} n_{j}\right) \mathrm{d} x, d, n \in \mathrm{H}^{1} .
$$

It is clear that $\hat{a}$ is continuous, and hence, by Riesz representation lemma, there exists a unique bounded linear operator $\hat{\mathcal{A}}: \mathrm{H}^{1} \rightarrow\left(\mathrm{H}^{1}\right)^{*}$ such that ${ }_{\left(\mathrm{H}^{1}\right)^{*}}\langle\hat{\mathcal{A}} d, n\rangle_{\mathrm{H}^{1}}=\hat{a}(d, n)$, for $d, n \in \mathrm{H}^{1}$. Next, we define an unbounded linear operator $\hat{\mathrm{A}}$ in $L^{2}$ as follows:

$$
\left\{\begin{array}{l}
D(\hat{\mathrm{A}})=\left\{d \in \mathrm{H}^{1}: \hat{\mathrm{A}} d \in L^{2}\right\} \\
\hat{\mathrm{A}}=\hat{\mathcal{A}} d, d \in D(\hat{\mathrm{A}}) .
\end{array}\right.
$$


Under our assumption on $\Omega$, it is known, see, for instance, [38, Section2, p. 65], that $\hat{\mathrm{A}}$ and $D(\hat{\mathrm{A}})$ can be characterized by

$$
\begin{cases}D(\hat{\mathrm{A}}) & :=\left\{d \in \mathbb{H}^{2}: \frac{\partial d}{\partial v}=0 \text { on } \partial \Omega\right\}, \\ \hat{\mathrm{A}} d & :=-\Delta d, \quad d \in D(\hat{\mathrm{A}}),\end{cases}
$$

where $v=\left(v_{1}, v_{2}, v_{3}\right)$ is the unit outward normal vector field on $\partial \Omega$ and $\frac{\partial d}{\partial v}$ is the directional derivative of $d$ in the direction $v$.

Let us recall that the operator $\hat{\mathrm{A}}$ is self-adjoint and nonnegative and $D\left(\hat{\mathrm{A}}^{1 / 2}\right)$ when endowed with the graph norm coincides with $\mathrm{H}^{1}$. Moreover, the operator $(I+\hat{\mathrm{A}})^{-1}$ is compact. Furthermore, if we denote

$$
\hat{\mathrm{V}}:=D\left(\hat{\mathrm{A}}^{1 / 2}\right)
$$

the $\left(\hat{\mathrm{V}}, L^{2}, \mathrm{~V}^{*}\right)$ is a Gelfand triple and

$$
\hat{\mathrm{V}}^{*}\left\langle d_{1}, d_{2}\right\rangle_{\hat{\mathrm{V}}}=\int_{\Omega} d_{1}(x) d_{2}(x) \mathrm{d} x
$$

\subsection{An abstract formulation of problem (1.1)}

With the notations we have introduced above, we can now rewrite problem (1.2) as an abstract equations. In fact by projecting the first equation in (2.13) onto $\mathrm{H}$ we obtain the following system

$$
\left\{\begin{array}{l}
\dot{v}+\mathrm{A} v=-B(v, v)-\Pi(\operatorname{div}[\nabla d \odot \nabla d])+\Pi f \\
\partial_{t} d+\hat{\mathrm{A}} d=|\nabla d|^{2} d-v \cdot \nabla d-\phi^{\prime}(d)+\left(\phi^{\prime}(d), d\right) d+d \times g, \\
|d|=1 \\
(v, d)(0)=\left(v_{0}, d_{0}\right)
\end{array}\right.
$$

\section{The existence and the uniqueness of a regular solution to problem (2.13)}

Throughout the whole section, we fix a map $\phi: \mathbb{R}^{3} \rightarrow \mathbb{R}^{3}$ satisfying the following set of conditions.

Assumption 3.1. The map $\phi: \mathbb{R}^{3} \rightarrow \mathbb{R}^{3}$ is of class $C^{2}$ and there exist constants $M_{0}>0, M_{1}>0$ and $M_{2}>0$ such that for all $n, d \in \mathbb{R}^{3}$

$$
\begin{aligned}
\left|\phi^{\prime}(n)\right| & \leq M_{0}(1+|n|), \\
\left|\phi^{\prime \prime}(n)-\phi^{\prime \prime}(d)\right| & \leq M_{1}|n-d|, \\
\left|\phi^{\prime \prime}(n)\right| & \leq M_{2} .
\end{aligned}
$$

Example 3.2. Let $H \in \mathbb{R}^{3}$ be a constant vector. Then the anisotropy energy potential $\phi$ due to the action of a magnetic or electric is defined by 


$$
\phi(d)=\frac{1}{2}\left[|H|^{2}-(d \cdot H)^{2}\right], d \in \mathbb{R}^{3} .
$$

This potential $\phi$ satisfies Assumption 3.1. In this case $H$ represents a constant magnetic or electric field applied to the nematic liquid crystal.

Another mathematical example which satisfies Assumption 3.1 is the potential defined by

$$
\phi(d)=\frac{1}{2}|d-\xi|^{2}, d \in \mathbb{R}^{3},
$$

where $\xi \in \mathbb{R}^{3}$ is a fixed constant vector.

Next, we consider the problem (2.13) on a finite time horizon $[0, T]$. Throughout the paper we put

$$
\mathcal{M}=\left\{d: \Omega \rightarrow \mathbb{R}^{3}:|d(x)|=1 \text { Leb-a.e. }\right\},
$$

In this section, we impose the following set of conditions on the data.

Assumption 3.3. We assume that $(f, g) \in L^{2}\left(0, T ; L^{2} \times D\left(\hat{\mathrm{A}}^{1 / 2}\right)\right)$ and $\left(v_{0}, d_{0}\right) \in$ $\mathrm{V} \times(D(\hat{\mathrm{A}}) \cap \mathcal{M})$.

Under this assumption we will prove in this section that Problem (2.13) has a unique local regular solution. Before stating and proving this result we define what we mean by a maximal local regular solution.

Definition 3.4. Let $T_{0} \in(0, T]$. A function $(v, d):\left[0, T_{0}\right] \rightarrow \mathrm{V} \times D(\hat{\mathrm{A}})$ is called a local regular solution to Problem (2.13) with initial data $\left(v_{0}, d_{0}\right)$ iff

(1) $(v, d) \in C\left(\left[0, T_{0}\right] ; \mathrm{V} \times D(\hat{\mathrm{A}})\right) \cap L^{2}\left(0, T_{0} ; D(\mathrm{~A}) \times D\left(\hat{\mathrm{A}}^{3 / 2}\right)\right)$,

(2) for all $t \in\left[0, T_{0}\right]$ the integral equations

$$
\begin{aligned}
v(t)= & v_{0}+\int_{0}^{t}[-\mathrm{A} v(s)-B(v(s), v(s))-\Pi(\operatorname{div}[\nabla d(s) \odot \nabla d(s)])] \mathrm{d} s+\int_{0}^{t} f(s) \mathrm{d} s, \\
d(t)= & d_{0}+\int_{0}^{t}\left[-\hat{\mathrm{A}} d(s)+|\nabla d(s)|^{2} d(s)-v(s) \cdot \nabla d(s)-\phi^{\prime}(d(s))\right. \\
& \left.+\left(\phi^{\prime}(d(s)) \cdot d(s)\right) d(s)\right] \mathrm{d} s \\
& +\int_{0}^{t}(d(s) \times g(s)) \mathrm{d} s
\end{aligned}
$$

hold in $\mathrm{H}$ and $D\left(\hat{\mathrm{A}}^{1 / 2}\right)$, respectively.

(3) For all $t \in\left[0, T_{0}\right] d(t) \in \mathcal{M}$.

(4) $\left(\partial_{t} v, \partial_{t} d\right) \in L^{2}\left(0, T_{0} ; \mathrm{H} \times D\left(\hat{\mathrm{A}}^{1 / 2}\right)\right)$.

Throughout this paper we will denote by $\left((v, d) ; T_{0}\right)$ a local regular solution defined on $\left[0, T_{0}\right]$.

We also need the definition of a maximal local regular solution. 
Definition 3.5. A pair $\left((v, d) ; T_{0}\right)$ with $T_{0} \in(0, T)$ and $(v, d):\left[0, T_{0}\right) \rightarrow \mathrm{V} \times D(\hat{\mathrm{A}})$ is called a maximal local regular solution to (2.13) with initial data $(v(0), d(0))=$ $\left(v_{0}, d_{0}\right)$ if

(1) $\left((v, d) ; T_{0}\right)$ defined on $\left[0, T_{0}\right)$ is a local regular solution to (2.13),

(2) for any other local regular solution $\left((\tilde{v}, \tilde{d}) ; \tilde{T}_{0}\right)$ we have

$$
\tilde{T}_{0} \leq T_{0} \text { and }(v, d)_{\left.\right|_{\left[0, \tilde{T}_{0}\right)}}=(\tilde{v}, \tilde{d})
$$

We state the following important remark.

Remark 3.6. Let

$$
\begin{aligned}
F(v, d): & =-B(v, v)-\Pi(\operatorname{div}[\nabla d \odot \nabla d]), \\
\tilde{G}(v, d) & =|\nabla d|^{2} d-v \cdot \nabla d-\phi^{\prime}(d)+\left(\phi^{\prime}(d) \cdot d\right) d, \\
G(v, d): & =|\nabla d|^{2} d-v \cdot \nabla d-\phi^{\prime}(d)+\left(\phi^{\prime}(d) \cdot d\right) d+d \times g .
\end{aligned}
$$

Then, the condition (4) of Definition 3.4 is equivalent to the following

$$
(F(v, d), G(v, d)) \in L^{2}\left(0, T_{0} ; \mathrm{H} \times D\left(\hat{\mathrm{A}}^{1 / 2}\right)\right) .
$$

We will see in Lemma 3.9 that if $(v, d) \in C\left(\left[0, T_{0}\right] ; \mathrm{V} \times D(\hat{\mathrm{A}})\right) \cap L^{2}\left(0, T_{0} ; D(A) \times\right.$ $\left.D\left(\hat{\mathrm{A}}^{3 / 2}\right)\right)$, then $(F(v, d), G(v, d)) \in L^{2}\left(0, T_{0} ; H \times D\left(\hat{\mathrm{A}}^{1 / 2}\right)\right)$.

With the definitions and remark in mind we are now ready to formulate our first result.

Theorem 3.7. Let $R_{1}>0, R_{2}>0$ and $g \in L^{2}\left(0, T ; D\left(\hat{\mathrm{A}}^{1 / 2}\right)\right)$. Then, there exist $T_{1}(g)>0$ and $T_{2}\left(R_{1}, R_{2}\right)>0$ such that the following holds.

If $\left(v_{0}, d_{0}\right) \in \mathrm{V} \times(D(\hat{\mathrm{A}}) \cap \mathcal{M})$ and $(f, g) \in L^{2}\left(0, T ; L^{2} \times D\left(\hat{\mathrm{A}}^{1 / 2}\right)\right)$ are such that

$$
\left[\left|v_{0}\right|_{V}^{2}+\left|d_{0}\right|_{D(\hat{\mathrm{A}})}^{2}\right]^{\frac{1}{2}} \leq R_{1} \text { and }\left[\int_{0}^{T}\left(|f(s)|_{L^{2}}^{2}+|g(s)|_{D\left(\hat{\mathrm{A}}^{1 / 2}\right)}^{2}\right) \mathrm{d} s\right]^{\frac{1}{2}} \leq R_{2},
$$

then the problem (2.13) has a local regular solution $\left((v, d) ; T_{0}\right)$ with $T_{0}=T_{1}(g) \wedge$ $T_{2}\left(R_{1}, R_{2}\right)$

Moreover, if $\left((u, n) ; T_{0}\right)$ is another local regular solution, then

$$
(u(t), n(t))=(v(t), d(t)) \text { for all } t \in\left[0, T_{0}\right] .
$$

In order to prove this theorem we shall introduce the following spaces

$$
\begin{aligned}
& \mathbf{X}_{T}^{1}=C([0, T] ; \mathrm{V}) \cap L^{2}(0, T ; D(\mathrm{~A})), \\
& \mathbf{X}_{T}^{2}=C([0, T] ; D(\hat{\mathrm{A}})) \cap L^{2}\left(0, T ; D\left(\hat{\mathrm{A}}^{\frac{3}{2}}\right)\right), \\
& \mathbf{Y}_{T}^{1}=L^{2}(0, T ; \mathrm{H}) \\
& \mathbf{Y}_{T}^{2}=L^{2}\left(0, T ; D\left(\hat{\mathrm{A}}^{\frac{1}{2}}\right)\right)
\end{aligned}
$$


We also set

$$
\begin{aligned}
& \mathbf{X}_{T}=\mathbf{X}_{T}^{1} \times \mathbf{X}_{T}^{2}, \\
& \mathbf{Y}_{T}=\mathbf{Y}_{T}^{1} \times \mathbf{Y}_{T}^{2} .
\end{aligned}
$$

Let $(v, n) \in \mathbf{X}_{T}$ and consider the following decoupled linear problem

$$
\left(\begin{array}{c}
\partial_{t} u \\
\partial_{t} d
\end{array}\right)+\left(\begin{array}{c}
\mathrm{A} u \\
\hat{\mathrm{A}} d
\end{array}\right)=\left(\begin{array}{c}
F(v, n)+f \\
G(v, n)+d \times g
\end{array}\right) .
$$

Before continuing further, let us recall the following result.

Lemma 3.8. If $\left(u_{0}, d_{0}\right) \in \mathrm{V} \times D(\hat{\mathrm{A}}),(\mathfrak{f}, \mathfrak{g}) \in L^{2}\left(0, T ; \mathrm{H} \times D\left(\hat{\mathrm{A}}^{\frac{1}{2}}\right)\right)$ and $T>0$, then the problem

$$
\left(\begin{array}{l}
\partial_{t} u \\
\partial_{t} d
\end{array}\right)+\left(\begin{array}{l}
\mathrm{A} u \\
\hat{\mathrm{A}} d
\end{array}\right)=\left(\begin{array}{l}
\mathfrak{f} \\
\mathfrak{g}
\end{array}\right)
$$

has a unique strong solution $(u, d) \in \mathbf{X}_{T}$. Moreover, there exists a constant $C>0$, independent of $T$, such that

$$
|(u, d)|_{\mathbf{X}_{T}}^{2} \leq C\left|\left(u_{0}, d_{0}\right)\right|_{\mathrm{V} \times D(\hat{\mathrm{A}})}^{2}+C|(\mathfrak{f}, \mathfrak{g})|_{L^{2}\left(0, T ; \mathrm{H} \times D\left(\hat{\mathrm{A}}^{\frac{1}{2}}\right)\right)}^{2} .
$$

Now we state the following lemma whose proof will be given in "Appendix."

Lemma 3.9. There exists a constant $C_{0}>0$, independent of $T$, such that for all $v_{i} \in \mathbf{X}_{T}^{1}, d_{i} \in \mathbf{X}_{T}^{2}, i=1,2$, the following inequalities hold

$$
\begin{aligned}
\left|F\left(v_{1}, n_{1}\right)-F\left(v_{2}, n_{2}\right)\right|_{L^{2}(0, T ; \mathrm{H})}^{2} \leq & C_{0} T^{\frac{1}{2}}\left|\left(v_{1}, n_{1}\right)-\left(v_{2}, n_{2}\right)\right|_{\mathbf{X}_{T}}^{2}\left[\left|\left(v_{1}, n_{1}\right)\right|_{\mathbf{X}_{T}}^{2}\right. \\
& \left.+\left|\left(v_{2}, n_{2}\right)\right|_{\mathbf{X}_{T}}^{2}\right] \\
\left|\tilde{G}\left(v_{1}, n_{1}\right)-\tilde{G}\left(v_{2}, n_{2}\right)\right|_{L^{2}\left(0, T ; D\left(\hat{\mathrm{A}}^{\frac{1}{2}}\right)\right)}^{2} \leq & C_{0}\left(T \vee T^{\frac{1}{2}}\right) \mid\left(v_{1}, n_{1}\right) \\
& -\left.\left(v_{2}, n_{2}\right)\right|_{\mathbf{X}_{T}} ^{2}\left[1+\sum_{i=1}^{2}\left|\left(v_{i}, n_{i}\right)\right|_{\mathbf{X}_{T}}^{6}\right] \\
\left|n_{1} \times g-n_{2} \times g\right|_{L^{2}\left(0, T ; \mathrm{H}^{1}\right)}^{2} \leq & C_{0}\left|n_{1}-n_{2}\right|_{\mathbf{X}_{T}^{2}}^{2}|g|_{L^{2}\left(0, T ; \mathrm{H}^{1}\right)}^{2}
\end{aligned}
$$

Now, we will give the proof of Theorem 3.7.

Proof of Theorem 3.7. Let $\Psi: \mathbf{X}_{T} \rightarrow \mathbf{X}_{T}$ be the map defined as follows. If $(v, n) \in$ $\mathbf{X}_{T}$, then $\Psi(v, n)=(u, d)$ iff $(u, d)$ is the unique regular solution to (3.10) with right hand side of the form

$$
\left(\begin{array}{l}
\mathfrak{f} \\
\mathfrak{g}
\end{array}\right)=\left(\begin{array}{c}
F(v, n)+f \\
\tilde{G}(v, n)+n \times g
\end{array}\right) .
$$


Let us observe that by Lemma 3.9 the term $(\mathfrak{f}, \mathfrak{g})$ defined in (3.14) belongs to $L^{2}\left(0, T ; \mathrm{H} \times D\left(\hat{\mathrm{A}}^{\frac{1}{2}}\right)\right)$. Hence, by Lemma $3.8(u, d) \in \mathbf{X}_{T}$, and so the map $\Psi$ is well-defined.

Now, let $R_{1}>0, R_{2}>0,(f, g) \in L^{2}\left(0, T ; H \times D\left(\hat{\mathrm{A}}^{1 / 2}\right)\right)$ and $\left(v_{0}, d_{0}\right) \in$ $\mathrm{V} \times(D(\hat{\mathrm{A}}) \cap \mathcal{M})$. Let

$$
\mathbf{K}_{R_{1}, R_{2}}=\left\{(v, n) \in \mathbf{X}_{T}:|(v, n)|_{\mathbf{X}_{T}}^{2} \leq R_{1}^{2}+R_{2}^{2}\right\} .
$$

Let $\left(v_{i}, n_{i}\right) \in \mathbf{K}_{R_{1}, R_{2}}$ and $\left(u_{i}, d_{i}\right)=\Psi\left(v_{i}, n_{i}\right), i=1,2$. Put $(u, d)=\left(u_{1}-u_{2}, d_{1}-\right.$ $\left.d_{2}\right)$. Then, it is easy to check that $(u, d)$ solves the following problem

$$
\left(\begin{array}{c}
\partial_{t} u \\
\partial_{t} d
\end{array}\right)+\left(\begin{array}{c}
\mathrm{A} u \\
\hat{\mathrm{A}} d
\end{array}\right)=\left(\begin{array}{c}
F\left(v_{1}, n_{1}\right)-F\left(v_{2}, n_{2}\right) \\
\tilde{G}\left(v_{1}, n_{1}\right)-\tilde{G}\left(v_{1}, n_{2}\right)+\left(n_{1}-n_{2}\right) \times g
\end{array}\right) .
$$

Hence, by Lemma 3.8 there exists a constant $C>0$, independent of $T$, such that

$$
\begin{array}{rl}
|(u, d)|_{\mathbf{X}_{T} \leq}^{2} & C\left(\left|F\left(v_{1}, n_{1}\right)-F\left(v_{2}, n_{2}\right)\right|_{L^{2}(0, T ; \mathrm{H})}^{2}+\left|\tilde{G}\left(v_{1}, n_{1}\right)-\tilde{G}\left(v_{2}, n_{2}\right)\right|_{L^{2}\left(0, T ; \mathrm{H}^{1}\right)}^{2}\right. \\
& \left.+\left|\left(n_{1}-n_{2}\right) \times g\right|_{L^{2}\left(0, T ; \mathrm{H}^{1}\right)}^{2}\right) .
\end{array}
$$

Then, by plugging (3.11), (3.12) and (3.13) in the above inequality and performing elementary calculations imply that there exists a constant $C_{1}>0$, independent of $T$, such that for all $\left(v_{i}, n_{i}\right) \in \mathbf{K}_{R_{1}, R_{2}}, i=1,2$,

$$
\begin{aligned}
& \left|\Psi\left(v_{1}, n_{1}\right)-\Psi\left(v_{2}, n_{2}\right)\right|_{\mathbf{X}_{T}}^{2} \leq C_{1}\left|\left(v_{1}, n_{1}\right)-\left(v_{2}, n_{2}\right)\right|_{\mathbf{X}_{T}}^{2}\left(\left[1+R_{1}^{6}+R_{2}^{6}\right]\left(T \vee T^{\frac{1}{2}}\right)\right. \\
& \left.\quad+\int_{0}^{T}|g(s)|_{\mathrm{H}^{1}}^{2} \mathrm{~d} s\right) .
\end{aligned}
$$

Since $g \in L^{2}\left(0, T ; \mathrm{H}^{1}\right)$, for any $\varepsilon>0$ there exists $T_{1} \in(0, T)$ such that

$$
C_{1} \int_{0}^{T_{1}}|g(s)|_{\mathrm{H}^{1}}^{2} \leq \varepsilon .
$$

Next, we choose a number $T_{2}$ such that

$$
C_{1}\left[1+R_{1}^{6}+R_{2}^{6}\right]\left(T_{2} \vee T_{2}^{\frac{1}{2}}\right) \leq \frac{1}{4} .
$$

Hence, by choosing $\varepsilon=\frac{1}{4}$ and setting $T_{0}=T_{1} \wedge T_{2}$ we infer that for all $\left(v_{i}, n_{i}\right) \in$ $\mathbf{K}_{R_{1}, R_{2}}, i=1,2$,

$$
\left|\Psi\left(v_{1}, n_{1}\right)-\Psi\left(v_{2}, n_{2}\right)\right|_{\mathbf{X}_{T}}^{2} \leq \frac{1}{2} .
$$

Hence, $\Psi$ has a unique fixed point $(u, d) \in \mathbf{X}_{T_{0}}$ satisfying

$$
\left(\begin{array}{c}
\partial_{t} u \\
\partial_{t} d
\end{array}\right)+\left(\begin{array}{c}
\mathrm{A} u \\
\hat{\mathrm{A}} d
\end{array}\right)=\left(\begin{array}{c}
F(u, d)+f \\
G(u, d)+d \times g
\end{array}\right) .
$$


Thus, in order to prove Theorem 3.7 it remains to prove that

$$
d(t) \in \mathcal{M} \text { for all } t \in\left[0, T_{0}\right]
$$

For this purpose, let

$$
z(t)=|d(t)|^{2}-1, t \in\left[0, T_{0}\right]
$$

We recall that there exists a constant $C>0$ such that for all $n \in \mathrm{H}^{3}$

$$
|n|_{\mathrm{H}^{\frac{5}{2}}} \leq C|n|_{\mathrm{H}^{2}}^{\frac{1}{2}}|n|_{\mathrm{H}^{3}}^{\frac{1}{2}} \text {. }
$$

Hence, since $d \in \mathbf{X}_{T_{0}}^{2}:=C\left(\left[0, T_{0}\right] ; D(\hat{\mathrm{A}})\right) \cap L^{2}\left(0, T ; D\left(\hat{\mathrm{A}}^{\frac{3}{2}}\right)\right), D\left(\hat{\mathrm{A}}^{\theta}\right) \subset \mathrm{H}^{2 \theta}$ and $\mathrm{H}^{2 \theta}, \theta>\frac{1}{2}$ is an algebra, by using the interpolation inequality (3.19) we easily show that

$$
z \in C\left(\left[0, T_{0}\right] ; \mathrm{H}^{2}\right) \cap L^{2}\left(0, T_{0} ; \mathrm{H}^{\frac{5}{2}}\right)
$$

Also, since $(u, d) \in X_{T_{0}}$ we infer from Lemma 3.9 that

$$
\partial_{t} d=-\hat{\mathrm{A}} d+\tilde{G}(u, d)+d \times g \in L^{2}\left(0, T_{0} ; \mathrm{H}^{1}\right) .
$$

Using this and $d \in \mathbf{X}_{T_{0}}^{2}$ we easily prove that

$$
\partial_{t} z \in L^{2}\left(0, T_{0} ; \mathrm{H}^{1}\right) .
$$

Now we will claim that $z$ satisfies the weak form of the following problem

$$
\left\{\begin{array}{l}
\partial_{t} z-\Delta z+u \cdot \nabla z=2|\nabla d|^{2} z-2\left(\phi^{\prime}(d) . d\right) z \\
\left.\frac{\partial z}{\partial v}\right|_{\partial \Omega}=0, \\
z(0)=0 .
\end{array}\right.
$$

Toward this end let $\varphi \in H^{1}(\Omega ; \mathbb{R})$ and fix $t \in\left[0, T_{0}\right]$. Since $d \in C\left(\left[0, T_{0}\right] ; D(\hat{\mathrm{A}})\right) \cap$ $L^{2}\left(0, T ; D\left(\hat{\mathrm{A}}^{\frac{3}{2}}\right)\right)$ and $D(\hat{\mathrm{A}}) \subset L^{\infty}$ we easily prove that $\varphi d \in C\left(\left[0, T_{0}\right] ; \mathrm{H}^{1}\right) \subset$ $L^{2}\left(0, T_{0} ; \mathrm{H}^{1}\right)$. Also, since $(u, d) \in X_{T_{0}}$ we infer from Lemma 3.9 that

$$
\partial_{t} d=-\hat{\mathrm{A}} d+\tilde{G}(u, d)+d \times g \in L^{2}\left(0, T_{0} ; \mathrm{H}^{1}\right) .
$$

Hence, in view of the Lions-Magenes lemma, see [37, Lemma III.1.2], we have

$$
\begin{aligned}
\left.\frac{1}{2} \frac{\mathrm{d}}{\mathrm{d} t} \int_{\Omega} \varphi(x) \mid d(t, x)\right)\left.\right|^{2} \mathrm{~d} x= & \left\langle\partial_{t} d(t), \varphi d(t)\right\rangle \\
= & -\int_{\Omega} \varphi(x) \mathrm{A} d(t, x) \cdot d(t, x) \mathrm{d} x \\
& -\int_{\Omega} \varphi(x)[u(t, x) \cdot \nabla d(t, x)] \cdot d(t, x) \mathrm{d} x
\end{aligned}
$$




$$
\begin{aligned}
& +\int_{\Omega} \varphi(x)|\nabla d(t, x)|^{2}|d(t, x)|^{2} \mathrm{~d} x \\
& -\int_{\Omega} \varphi\left(|d(t, x)|^{2}-1\right)\left(\phi^{\prime}(d(t, x)) \cdot d(t, x)\right) \mathrm{d} x,
\end{aligned}
$$

where we used the fact that $\varphi d \perp_{\mathbb{R}^{3}} d \times g$. Since $d(t) \in D(\hat{\mathrm{A}})$ and $\varphi d(t) \in \mathrm{H}^{1}$ for all $t \in\left[0, T_{0}\right]$, by using [3, Equation (2.6)] we infer that

$$
-\int_{\Omega} \hat{\mathrm{A}} d(t, x) \cdot \varphi(x) d(t, x) \mathrm{d} x=-\int_{\Omega}(\nabla d(t, x))(\nabla[\varphi(x) d(t, x)]) \mathrm{d} x .
$$

Thus, straightforward calculation yields

$$
-\int_{\Omega} \hat{\mathrm{A}} d(t, x) \cdot \varphi(x) d(t, x) \mathrm{d} x=-\int_{\Omega} \varphi(x)|\nabla d(t, x)|^{2} \mathrm{~d} x-\frac{1}{2} \int_{\Omega} \varphi(x) \nabla|d(t, x)|^{2} \mathrm{~d} x .
$$

Hence, recalling the definition of $z$ and using the last identity in (3.24) implies

$$
\begin{gathered}
\frac{1}{2} \int_{\Omega} \partial_{t} z(t, x) \varphi(x) \mathrm{d} x+\frac{1}{2} \int_{\Omega} \nabla z(t, x) \nabla \varphi(x) \mathrm{d} x+\frac{1}{2} \int_{\Omega} u(t, x) \nabla z(t, x) \varphi(x) \mathrm{d} x \\
=\int_{\Omega}|\nabla d(t, x)|^{2} z(t, x) \varphi(x) \mathrm{d} x-\int_{\Omega} z(t, x)\left(\phi^{\prime}(d(t, x)) \cdot d(t, x)\right) \varphi(x) \mathrm{d} x .
\end{gathered}
$$

This is exactly the weak form of (3.23).

By Proposition B.3 $z$ is the unique solution of (3.23) and satisfies

$$
\sup _{0 \leq t \leq T}|z(t)|_{L^{2}}^{2}+\int_{0}^{T}|\nabla z(t)|^{2} \mathrm{~d} t \leq|z(0)|_{L^{2}}^{2} e^{c \int_{0}^{T}\left[|\nabla d|_{L^{4}}^{4}+\left(1+|d|_{H^{2}}^{2}\right)\right] \mathrm{d} t} .
$$

Since $z(0)=0$, we infer that

$$
\sup _{0 \leq t \leq T}|z(t)|_{L^{2}}^{2}=\sup _{0 \leq t \leq T} \int_{\Omega}\left(|d|^{2}-1\right)^{2} \mathrm{~d} x=0,
$$

which implies that $d(t) \in \mathcal{M}$ for all $t \in\left[0, T_{0}\right]$. This completes the proof of (3.18). This also completes the proof of Theorem 3.7.

\section{The existence and the uniqueness of a maximal local regular solution to (2.13)}

The aim of this section is to prove that Problem (2.13) has a unique maximal local regular solution when the initial data has small energy. The main result of the section is Proposition 4.3, and it is a generalization of [26, Lemma 5.2]. Before proceeding to a precise statement and a detailed proof of the result let us introduce few notations. For $R>0$ and $(u, n) \in \mathrm{H} \times \mathrm{H}^{1}$ we set

$$
\mathcal{E}_{R}(u, n):=\frac{1}{2} \sup _{x \in \Omega} \int_{B(x, 2 R)}\left(|u(y)|^{2}+|\nabla n(y)|^{2}+2 \phi(n(y))\right) \mathrm{d} y,
$$


and

$$
\mathcal{E}(u, n):=\frac{1}{2}\left(|u|_{\mathrm{H}}^{2}+|\nabla n|_{L^{2}}^{2}\right)+\int_{\Omega} \phi(n(x)) \mathrm{d} x .
$$

We also recall the following important lemma, see [36, Lemma 3.1\& 3.2].

Lemma 4.1. There exist $c_{1}>0$ and $r_{0}>0$ such that for every $h \in L^{\infty}\left(0, T ; L^{2}\right) \cap$ $L^{2}\left(0, T ; \mathrm{H}^{1}\right)$ we have

$$
\begin{aligned}
\int_{0}^{T}|h(t)|_{L^{4}}^{4} \mathrm{~d} t \leq & c_{1}\left(\sup _{(t, x) \in[0, T] \times \Omega} \int_{B\left(x, r_{0}\right)}|h(t, y)|^{2} \mathrm{~d} y\right) \\
& \times\left(\int_{0}^{T}|\nabla h(t)|_{L^{2}}^{2} \mathrm{~d} t+\frac{1}{r_{0}^{2}} \int_{0}^{T}|h(t)|_{L^{2}}^{2} \mathrm{~d} t\right) .
\end{aligned}
$$

Remark 4.2. Let $r_{0}>0$ be as in Lemma 4.1. In view of 4.3 and [33, Theorem 3.4], we infer that there exists $c_{2}>0$ such that for all $h \in L^{\infty}\left(0, T ; \mathrm{H}^{1}\right) \cap L^{2}(0, T ; D(\hat{\mathrm{A}}))$ we have

$$
\begin{aligned}
\int_{0}^{T}|\nabla h(t)|_{L^{4}}^{4} \leq & c_{2}\left(\sup _{(t, x) \in[0, T] \times \Omega} \int_{B\left(x, r_{0}\right)}|\nabla h(t, y)|^{2} \mathrm{~d} y\right)\left(\int_{0}^{T}|\Delta h(t)|_{L^{2}}^{2} \mathrm{~d} t\right. \\
& \left.+\frac{1}{r_{0}^{2}} \int_{0}^{T}|\nabla h(t)|_{L^{2}}^{2} \mathrm{~d} t\right) .
\end{aligned}
$$

We state and prove the following important result.

Proposition 4.3. There exist a constant $\varepsilon_{0}>0$ and a function

$$
\theta_{0}:\left(0, \varepsilon_{0}\right) \times(0, \infty) \rightarrow(0, \infty),
$$

which is non-increasing w.r.t. the second variable and non-decreasing w.r.t. the first one, such that the following holds:

Let $r_{0}>0$ be as in Lemma 4.1. Let $(f, g) \in L^{2}\left(0, T ; \mathrm{H} \times D\left(\hat{\mathrm{A}}^{1 / 2}\right)\right),\left(v_{0}, d_{0}\right) \in$ $\mathrm{V} \times D(\hat{\mathrm{A}})$ and $R_{0} \in\left(0, r_{0}\right]$ are such that

$$
\mathcal{E}_{2 R_{0}}\left(v_{0}, d_{0}\right)<\varepsilon_{0}^{2}
$$

Then, there exists a unique maximal local regular solution $\left((v, d) ; T_{0}\right)$ to problem (2.13) satisfying

$$
\begin{gathered}
T_{0} \geq \frac{R_{0}^{2}}{\left(R_{0}^{\frac{1}{2}}+1\right)^{4}} \theta_{0}\left(\varepsilon_{1}, E_{0}\right), \\
\sup _{0 \leq t \leq T_{0}} \mathcal{E}_{R_{0}}(v(t), d(t)) \leq 2 \varepsilon_{1}^{2},
\end{gathered}
$$

where $E_{0}:=\mathcal{E}\left(v_{0}, d_{0}\right)$ and $\varepsilon_{1}^{2}=\mathcal{E}_{2 R_{0}}\left(v_{0}, d_{0}\right)$. 
Remark 4.4. In this theorem, the length $T_{0}$ is not the length of the existence interval but the length of the existence interval as long as the condition (4.7) is satisfied.

Note also that (4.7) is equivalent to

$$
\frac{1}{2} \sup _{0 \leq t \leq T_{0}} \sup _{x \in \Omega} \int_{B\left(x, R_{0}\right)}\left(|v(t, y)|^{2}+|\nabla d(t, y)|^{2}+2 \phi(d(t, y))\right) \mathrm{d} y \leq 2 \varepsilon_{1}^{2} .
$$

In order to prove the above proposition we need several results. For $n \in \mathbb{R}^{3}$

$$
\alpha(n)=\phi^{\prime}(n) \cdot n .
$$

We state and prove the following elementary results.

Claim 4.5. Let $u \in \mathrm{H}, n \in D(\hat{\mathrm{A}}) \cap \mathcal{M}$ and $m \in C\left(\left[0, T_{*}\right) ;(D(\hat{\mathrm{A}}) \cap \mathcal{M})\right)$ such that $\partial_{t} m \in L^{2}\left(0, T_{*} ; L^{2}\right)$. Then,

$$
\begin{aligned}
& \left\langle u \cdot \nabla n,|\nabla n|^{2} n-\phi^{\prime}(n)+\alpha(n) n\right\rangle=0, \\
& \left\langle\partial_{t} m,|\nabla m|^{2} m-\alpha(m) m\right\rangle=0 .
\end{aligned}
$$

Proof. Let us fix $u \in \mathrm{V}, n \in D(\hat{\mathrm{A}})$ and $m:\left[0, T_{*}\right) \rightarrow D(\hat{\mathrm{A}}) \cap \mathcal{M}$ satisfying the assumptions of Claim 4.5. Then, since $\operatorname{div} u=0$ and the fact $n(x) \in \mathbb{S}^{2} x$-a.e. we get

$$
\begin{aligned}
\left\langle u \cdot \nabla n,|\nabla n|^{2} n-\phi^{\prime}(n)+\alpha(n) n\right\rangle= & \frac{1}{2} \int_{\Omega} u(x) \cdot \nabla|n(x)|_{\mathbb{R}^{3}}^{2}\left(|\nabla n(x)|^{2}+\alpha(n(x))\right) \mathrm{d} x \\
& -\int_{\Omega} u(x) \cdot \nabla \phi(n(x)) \mathrm{d} x=0 .
\end{aligned}
$$

Since $m \in C\left(\left[0, T_{*}\right) ; D(\hat{\mathrm{A}}) \cap \mathcal{M}\right)$, we infer that for all $t \in\left[0, T_{*}\right)$

$$
\begin{aligned}
& \left\langle\partial_{t} m(t),|\nabla m(t)|^{2} m(t)-\alpha(m(t)) m(t)\right\rangle \\
& \quad=\frac{1}{2} \int_{\Omega} \partial_{t}|m(t, x)|_{\mathbb{R}^{3}}^{2}\left(|\nabla m(t, x)|^{2}-\alpha(m(t, x))\right) \mathrm{d} x=0,
\end{aligned}
$$

which completes the proof of the claim.

We also recall the following result, see [4, Eq. (2.10)].

Claim 4.6. For any $u \in \mathrm{H} \cap L^{4}$ and $n \in D(\hat{\mathrm{A}})$

$$
-\langle\operatorname{div}(\nabla n \odot \nabla n), u\rangle+\langle u \cdot \nabla n, \Delta n\rangle=0 .
$$

By Theorem 3.7, Problem (2.13) has a unique maximal local regular solution $(v, d) \in X_{T_{*}}$ provided that $\left(v_{0}, d_{0}\right) \in \mathrm{V} \times D(\hat{\mathrm{A}})$ and $(f, g) \in L^{2}\left(0, T ; L^{2} \times D\left(\hat{\mathrm{A}}^{1 / 2}\right)\right)$. Hereafter, we fix such a maximal local regular solution and we set,

$$
\mathcal{E}(v(t), d(t))=\frac{1}{2}\left(|v(t)|_{L^{2}}^{2}+|\nabla d(t)|_{L^{2}}^{2}\right)+\int_{\Omega} \phi(d(t, x)) \mathrm{d} x, \text { for } t \in\left[0, T_{*}\right], .
$$

We then prove the following important a priori estimates. 
Lemma 4.7. Let $\left(v_{0}, d_{0}\right) \in \mathrm{V} \times D(\hat{\mathrm{A}}),(f, g) \in L^{2}\left(0, T ; L^{2} \times \mathrm{H}^{1}\right)$ and $(v, d) \in X_{T_{*}}$ be a maximal local regular solution to the problem (2.13). Then for all $s, t \in\left[0, T_{*}\right]$ with $s \leq t$, the following inequality holds

$$
\begin{aligned}
& \mathcal{E}(v(t), d(t))+\frac{1}{2} \int_{s}^{t}\left(|\nabla v(r)|_{L^{2}}^{2}+|R(d(r))|_{L^{2}}^{2}\right) d r \leq \mathcal{E}(v(s), d(s)) \\
& \quad+\frac{1}{2} \int_{s}^{t}\left(|f(r)|_{\mathrm{H}^{-1}}^{2}+|g(r)|_{L^{2}}^{2}\right) d r
\end{aligned}
$$

where, for $n \in D(\hat{\mathrm{A}})$, we put

$$
\begin{aligned}
& \alpha(n)=\phi^{\prime}(n) \cdot n, \\
& R(n)=\Delta n+|\nabla n|^{2} n-\phi^{\prime}(n)+\alpha(n) .
\end{aligned}
$$

Proof. Since the maximal smooth solution $(v, d)$ satisfies part (1) of Definition 3.4, it is not difficult to show that $(v(t), R(d(t))) \in L^{2}\left(0, T_{*} ; D(\mathrm{~A}) \times D\left(\hat{\mathrm{A}}^{\frac{1}{2}}\right)\right)$. We also have $\left(\partial_{t} v, \partial_{t} d\right) \in L^{2}\left(0, T_{*} ; \mathrm{H} \times D\left(\hat{\mathrm{A}}^{\frac{1}{2}}\right)\right)$ because $(v, d)$ satisfies. Hence, by applying the Lions-Magenes lemma ([37, Lemma III.1.2]) and Claims 4.5 and 4.6 we infer that for all $t \in\left[0, T_{*}\right)$

$$
\begin{aligned}
-\left\langle\partial_{t} d(t), R(d(t))\right\rangle-\left\langle\partial_{t} v(t), \Delta v\right\rangle & =\frac{\mathrm{d}}{\mathrm{d} t} \mathcal{E}(v(t), d(t))+|\nabla v(t)|_{L^{2}}^{2}+|R(d(t))|_{L^{2}}^{2} \\
& =\langle f(t), v(t)\rangle+\langle d(t) \times g(t), R(d(t))\rangle .
\end{aligned}
$$

From the Cauchy-Schwarz and the Young inequalities and, the fact $d(t) \in \mathcal{M}, t \in$ $\left[0, T_{*}\right)$, which is part (4) of Definition 3.4, we infer that there exists a constant $C>0$ such that

$$
\begin{aligned}
& \frac{\mathrm{d}}{\mathrm{d} t} \mathcal{E}(v(t), d(t))+|\nabla v(t)|_{L^{2}}^{2}+|R(d(t))|_{L^{2}}^{2} \\
& \quad \leq C|f(t)|_{\mathrm{H}^{-1}}|\nabla v(t)|_{L^{2}}+C|d(t) \times g(t)|_{L^{2}}|R(d(t))|_{L^{2}} \\
& \quad \leq \frac{1}{2}\left(|\nabla v(t)|_{L^{2}}^{2}+|R(d(t))|_{L^{2}}^{2}\right)+\frac{1}{2}\left(|f(t)|_{\mathrm{H}^{-1}}^{2}+|g(t)|_{L^{2}}^{2}\right) .
\end{aligned}
$$

Absorbing the first term on the right hand side of the last inequality into the left hand side and integrating over $[s, t] \subset\left[0, T_{*}\right)$ completes the proof of the lemma.

For any $\varepsilon>0$ and $R>0$ we define the time

$$
T(\varepsilon, R)=\inf \left\{t \in\left[0, T_{*}\right): \mathcal{E}_{R}(v(t), d(t))>2 \varepsilon^{2}\right\} \wedge T_{*} .
$$

Remark 4.8. Let $\varepsilon>0$ and $R>0$. Then, for any $t \in[0, T(\varepsilon, R)]$

$$
\mathcal{E}_{R}(v(t), d(t)) \leq 2 \varepsilon^{2} \text {. }
$$

We state and prove the following lemma. 
Lemma 4.9. Let $\left(v_{0}, d_{0}\right) \in \mathrm{V} \times D(\hat{\mathrm{A}})$ and $(f, g) \in L^{2}\left(0, T ; L^{2} \times \mathrm{H}^{1}\right)$. There exist $\varepsilon_{0}>0$ and $K_{0}>0$ such that if $(v, d) \in X_{T_{*}}$ is a maximal local regular solution to the problem (2.13), then for all $\varepsilon \in\left(0, \varepsilon_{0}\right), R \in\left(0, r_{0}\right]$, where $r_{0}>0$ is the constant from Lemma 4.1, and for all $t \in[0, T(\varepsilon, R)]$

$$
\begin{aligned}
& \int_{0}^{t}|\Delta d(r)|_{L^{2}}^{2} d r \\
& \quad \leq K_{0}\left[E_{0}+\frac{1}{2} \int_{0}^{t}\left(|f(s)|_{\mathrm{H}^{-1}}^{2}+|g(s)|_{L^{2}}^{2}\right) \mathrm{d} s+\left(1+\frac{2 \varepsilon^{2}}{R^{2}}\right) t\right] \\
& \int_{0}^{t}\left(|v(s)|_{L^{4}}^{4}+|\nabla d(s)|_{L^{4}}^{4}\right) \mathrm{d} s \\
& \quad \leq K_{0} \varepsilon^{2}\left[E_{0}+\frac{1}{2} \int_{0}^{t}\left(|f(s)|_{\mathrm{H}^{-1}}^{2}+|g(s)|_{L^{2}}^{2}\right) \mathrm{d} s+\left(1+\frac{2 \varepsilon^{2}}{R^{2}}\right) t\right]
\end{aligned}
$$

Proof. Let $r_{0}>0$ be the constant from Lemma 4.1, $R \in\left[0, r_{0}\right]$ and $\varepsilon \in\left(0, \varepsilon_{0}\right)$ where $\varepsilon_{0}$ is number to be chosen later. We set

$$
E_{0}=\mathcal{E}\left(v_{0}, d_{0}\right)
$$

Since $\phi$ is twice continuously differentiable and the 2-sphere $\mathbb{S}^{2}$ is compact, we can and will assume throughout that for some constant $M>0$

$$
2\left|\phi^{\prime}(n)-\alpha(n) n\right|^{2} \leq M, n \in \mathbb{S}^{2}
$$

From this observation we infer that for all $t \in[0, T(\varepsilon, R)]$

$$
\begin{aligned}
\int_{0}^{t}|\Delta d(s)|_{L^{2}}^{2} \mathrm{~d} s \leq & 2 \int_{0}^{t}\left|\Delta d(s)-\phi^{\prime}(d(s))+\alpha(d(s)) d(s)\right|_{L^{2}}^{2} \mathrm{~d} s+M t \\
& \leq 4 \int_{0}^{t}|R(d(s))|_{L^{2}}^{2} \mathrm{~d} s+4 \int_{0}^{t}|\nabla d(s)|_{L^{4}}^{4} \mathrm{~d} s+M t .
\end{aligned}
$$

The last line of the above inequalities, (4.4), (4.9) and (4.11) imply that

$$
\begin{aligned}
\int_{0}^{t}|\Delta d(s)|_{L^{2}}^{2} \mathrm{~d} s \leq & 4 c_{2}\left(\sup _{(s, x) \in[0, t] \times \Omega} \int_{B(x, R)}|\nabla d(s, y)|^{2} \mathrm{~d} y\right)\left(\int_{0}^{t}|\Delta d(t)|_{L^{2}}^{2} \mathrm{~d} t\right. \\
& \left.+\frac{1}{R^{2}} \int_{0}^{t}|\nabla d(s)|_{L^{2}}^{2} \mathrm{~d} s\right) \\
& +4 \int_{0}^{t}|R(d(s))|_{L^{2}}^{2} \mathrm{~d} s \\
\leq & 4\left[E_{0}+\frac{1}{2} \int_{0}^{t}\left(|f(s)|_{\mathrm{H}^{-1}}^{2}+|g(s)|_{L^{2}}^{2}\right) \mathrm{d} s\right] \\
& +8 c_{2} \varepsilon^{2}\left(\int_{0}^{t}|\Delta d(s)|_{L^{2}}^{2} \mathrm{~d} s+\frac{2 \varepsilon^{2}}{R^{2}} t\right)+M t
\end{aligned}
$$


Now choosing $\varepsilon_{0}>0$ so that $1-8 c_{2} \varepsilon_{0}^{2} \geq \frac{1}{2}$, we infer that $1-8 c_{2} \varepsilon^{2}>\frac{1}{2}$ and for all $t \in[0, T(\varepsilon, R)]$

$\int_{0}^{t}|\Delta d(s)|_{L^{2}}^{2} \mathrm{~d} s \leq 8\left[E_{0}+\frac{1}{2} \int_{0}^{t}\left(|f(s)|_{\mathrm{H}^{-1}}^{2}+|g(s)|_{L^{2}}^{2}\right) \mathrm{d} s\right]+16 c_{2}\left(\frac{2 \varepsilon^{2}}{R^{2}}+M\right) t$,

which completes the proof of (4.12).

We now proceed to the proof of (4.13). For this we observe that by Lemma 4.1 and (4.12) we infer that for all $t \in[0, T(\varepsilon, R)]$

$$
\begin{aligned}
& \int_{0}^{t}|\nabla d(s)|_{L^{4}}^{4} \mathrm{~d} s \leq 4 c_{2} \varepsilon^{2}\left(\int_{0}^{t}|\Delta d(s)|_{L^{2}}^{2} \mathrm{~d} s+\frac{1}{R^{2}} \int_{0}^{t}|\nabla d(s)|_{L^{2}}^{2} \mathrm{~d} s\right) \\
& \quad \leq 4 c_{2} \varepsilon^{2}\left(K_{0}\left[E_{0}+\frac{1}{2} \int_{0}^{t}\left(|f(s)|_{\mathrm{H}^{-1}}^{2}+|g(s)|_{L^{2}}^{2}\right) \mathrm{d} s+\left(1+\frac{2 \varepsilon^{2}}{R^{2}}\right) t\right]+\frac{2 \varepsilon^{2}}{R^{2}} t\right) .
\end{aligned}
$$

In a similar way, we prove that for all $t \in[0, T(\varepsilon, R)]$

$$
\begin{aligned}
\int_{0}^{t}|v(s)|_{L^{4}}^{4} \mathrm{~d} s & \leq 4 c_{2} \varepsilon^{2}\left(\int_{0}^{t}|\nabla v(s)|_{L^{2}}^{2} \mathrm{~d} s+\frac{1}{r_{0}^{2}} \int_{0}^{t}|v(s)|_{L^{2}}^{2} \mathrm{~d} s\right) \\
& \leq 4 c_{2} \varepsilon^{2}\left(K_{0}\left[E_{0}+\frac{1}{2} \int_{0}^{t}\left(|f(s)|_{\mathrm{H}^{-1}}^{2}+|g(s)|_{L^{2}}^{2}\right) \mathrm{d} s\right]+\frac{2 \varepsilon^{2}}{R^{2}} t\right)
\end{aligned}
$$

which altogether with (4.14) imply (4.13).

We will need the following estimates which will be proved in "Appendix C."

Claim 4.10. There exists a constant $K_{1}>0$ such that for all $v \in D(A)$ and $n \in$ $D\left(\hat{\mathrm{A}}^{3 / 2}\right)$ we have

$$
\begin{aligned}
|\langle A v,-\Pi[\operatorname{div}(\nabla n \odot \nabla n)]\rangle| \leq & \frac{1}{12}\left(|A v|_{L^{2}}^{2}+|\nabla \Delta n|_{L^{2}}^{2}\right) \\
& +K_{1}|\nabla n|_{L^{4}}^{4}|\Delta n|_{L^{2}}^{2} \\
\left.\right|_{\left(\mathrm{H}^{1}\right)^{*}}\left\langle\hat{\mathrm{A}}^{2} n,(v \cdot \nabla n)\right\rangle_{\mathrm{H}^{1}} \mid \leq & \frac{1}{12}\left(|\nabla \Delta n|_{L^{2}}^{2}+|\mathrm{A} v|_{L^{2}}^{2}\right)+K_{1}|\nabla v|_{L^{2}}^{2}|(\nabla n)|_{L^{4}}^{2} \\
& +K_{1}\left[|v|_{L^{4}}^{2}+|v|_{L^{4}}^{4}\right]|\Delta n|_{L^{2}} \\
\left.\right|_{\left(\mathrm{H}^{1}\right)^{*}}\left\langle\hat{\mathrm{A}}^{2} n,\left(|\nabla n|^{2} n\right)\right\rangle_{\mathrm{H}^{1}} \mid \leq & \frac{1}{12}|\nabla \Delta n|_{L^{2}}^{2}+K_{1}\left(\left[|\nabla n|_{L^{4}}^{4}+|\nabla n|_{L^{4}}^{2}\right]|\Delta n|_{L^{2}}^{2}\right. \\
& \left.+|\nabla n|_{L^{4}}^{4}\left(|\nabla n|_{L^{2}}^{2}+|\Delta n|_{L^{2}}^{2}\right)\right)
\end{aligned}
$$

We will also need the following results.

Claim 4.11. There exists a constant $K_{2}>0$ such that for all $n \in D\left(\hat{\mathrm{A}}^{3 / 2}\right)$ we have

$$
\left.\left.\right|_{\left(\mathrm{H}^{1}\right)^{*}}\left\langle\hat{\mathrm{A}}^{2} n, \alpha(n) n-\phi^{\prime}(n)\right\rangle_{\mathrm{H}^{1}}\left|\leq \frac{1}{12}\right| \nabla \Delta n\right|_{L^{2}} ^{2}+K_{2}|\nabla n|_{L^{2}}^{2} \text {. }
$$


Proof. Using the Cauchy-Schwarz inequality and the fact that there exists a constant $M>0$ such that

$$
\left|\phi^{\prime}(n)\right|+\left|\phi^{\prime \prime}(n)\right| \leq M, n \in \mathbb{S}^{2}
$$

we infer that there exists a constant $C>0$ such that

$$
\begin{aligned}
\left.\right|_{\left(\mathrm{H}^{1}\right)^{*}}\left\langle\hat{\mathrm{A}}^{2} n, \alpha(n) n-\phi^{\prime}(n)\right\rangle_{\mathrm{H}^{1}} \mid \leq & |\nabla \Delta n|_{L^{2}}\left(|\nabla(\alpha(n) n)|_{L^{2}}+\left|\phi^{\prime \prime}(n) \nabla n\right|_{L^{2}}\right) \\
\leq & C|\nabla \Delta n|_{L^{2}}\left(|| \nabla n|| \phi^{\prime}(n)|| n||_{L^{2}}+\left.\left.|| \nabla n|| \phi^{\prime \prime}(n)|| n\right|^{2}\right|_{L^{2}}\right. \\
& \left.+\left|\phi^{\prime \prime}(n) \nabla n\right|_{L^{2}}\right) \\
\leq & C|\nabla \Delta n|_{L^{2}}|\nabla n|_{L^{2}} .
\end{aligned}
$$

We now complete the proof using the Young inequality in the last line.

Hereafter, we put for all $s, t \in[0, T]$ with $s \leq T$

$$
\begin{aligned}
& \Psi(s, t)=\frac{1}{2} \int_{s}^{t}\left(|f(r)|_{\mathrm{H}^{-1}}^{2}+|g(r)|_{L^{2}}^{2}\right) d r \text { and } \Psi(t)=\Psi(0, t), \\
& \Xi(s, t)=\frac{1}{2} \int_{s}^{t}\left(|f(r)|_{L^{2}}^{2}+|g(r)|_{\mathrm{H}^{1}}^{2}\right) d r \text { and } \Xi(t)=\Xi(0, t) \quad \forall s \leq t \in[0, T] .
\end{aligned}
$$

Lemma 4.12. Let $\left(v_{0}, d_{0}\right) \in \mathrm{V} \times D(\hat{\mathrm{A}}),(f, g) \in L^{2}\left(0, T ; L^{2} \times \mathrm{H}^{1}\right)$ and $(v, d) \in X_{T_{*}}$ be a maximal local regular solution to the problem (2.13). Let us put

$$
\Sigma_{0}=\left[E_{0}+\left|\Delta d_{0}\right|_{L^{2}}^{2}+\left|\nabla v_{0}\right|_{L^{2}}^{2}+\Psi(T)+\Xi(T)+\left(E_{0}+1\right) \Xi(T)\right]<\infty .
$$

Then, there exist constants $K_{3}>0$ and $K_{4}>0$ such that for all $\tau \in\left[0, T_{*}\right]$ we have

$$
\begin{aligned}
& \sup _{0 \leq s \leq \tau}\left(|\nabla v(s)|_{L^{2}}^{2}+|\Delta d(s)|_{L^{2}}^{2}\right)+2 \int_{0}^{\tau}\left(|\nabla \Delta d(s)|_{L^{2}}^{2}+|\mathrm{A} v(s)|_{L^{2}}^{2}\right) \mathrm{d} s \\
& \quad \leq K_{3} \Sigma_{0} e^{K_{4} \Xi(T)} e^{K_{4} \int_{0}^{\tau}\left[|\nabla d(r)|_{L^{4}}^{4}+|v(r)|_{L^{4}}^{4}+|\nabla d(r)|_{L^{4}}^{2}+|v(r)|_{L^{4}}^{2}\right] d r} .
\end{aligned}
$$

Proof. Throughout this proof $C>0$ will denote an universal constant which may change from one term to the other. Let $\left(v_{0}, d_{0}\right) \in \mathrm{H} \times D(\hat{\mathrm{A}})$ and $\left((v, d) ; T_{*}\right)$ be a local regular solution to the problem (2.13).

By part (1) and (4) of Definition 3.4 we have $(v, d) \in L^{2}\left(0, T_{*} ; D(\mathrm{~A}) \times D\left(\hat{\mathrm{A}}^{\frac{3}{2}}\right)\right)$ and $\left(\partial_{t} v, \partial d\right) \in L^{2}\left(0, T_{*} ; \mathrm{H} \times D\left(\hat{\mathrm{A}}^{\frac{1}{2}}\right)\right) \subset L^{2}\left(0, T_{*} ; \mathrm{V}^{*} \times D(\hat{\mathrm{A}})\right)$. Hence, by the Lions-Magenes lemma ([37, Lemma III.1.2]) we infer that

$$
\begin{aligned}
\frac{1}{2} \frac{\mathrm{d}}{\mathrm{d} t}\left(|\Delta d(t)|_{L^{2}}^{2}+|\nabla v(t)|_{L^{2}}^{2}\right) & =\left\langle\partial_{t} \Delta d(t), \Delta d(t)\right\rangle+\left\langle\partial_{t} \nabla v(t), \nabla v(t)\right\rangle \\
& ={ }_{\mathrm{H}^{1}}\left\langle\partial_{t} d(t), \hat{\mathrm{A}}^{2} d(t)\right\rangle_{\left(\mathrm{H}^{1}\right)^{*}}+\left(\partial_{t} v(t), A v(t)\right) .
\end{aligned}
$$


Hence we deduce that,

$$
\begin{aligned}
& \frac{1}{2} \frac{\mathrm{d}}{\mathrm{d} t}\left(|\Delta d(t)|_{L^{2}}^{2}+|\nabla v(t)|_{L^{2}}^{2}\right)=-|\nabla \Delta d(t)|_{L^{2}}^{2}-|\mathrm{A} v(t)|_{L^{2}}^{2}+\langle f(t), A v(t)\rangle \\
& -\langle\nabla(g(t) \times d(t)), \nabla \Delta d(t)\rangle \\
& +{ }_{\left(\mathrm{H}^{1}\right)^{*}}\left\langle\hat{\mathrm{A}}^{2} d(t),|\nabla d(t)|^{2} d(t)+\alpha(d(t)) d(t)\right. \\
& \left.-\phi^{\prime}(d(t))-v(t) \cdot \nabla d(t)\right\rangle_{\mathrm{H}^{1}} \\
& -\langle\operatorname{div}(\nabla d(t) \odot \nabla d(t))+v(t) \cdot \nabla v(t), A v(t)\rangle .
\end{aligned}
$$

By using the Cauchy-Schwarz, the Young inequalities and the Ladyzhenskaya inequality, see [37, Lemma III.3.3], we obtain

$$
\begin{aligned}
\langle v & \cdot \nabla v, \mathrm{~A} v\rangle \leq C|A v|_{L^{2}}|v|_{L^{4}}|\nabla v|_{L^{4}} \\
& \leq C|\mathrm{~A} v|^{\frac{3}{2}}|v|_{L^{4}}|\nabla v|_{L^{2}}^{\frac{1}{2}} \\
& \leq \frac{1}{4}|\mathrm{~A} v|^{2}+C|v|_{L^{4}}^{4}|\nabla v|_{L^{2}}^{2} .
\end{aligned}
$$

By using the Hölder and Young inequalities, and the Sobolev embedding $\mathrm{H}^{1} \hookrightarrow L^{4}$ we also have

$$
\begin{aligned}
\left(\mathrm{H}^{1}\right)^{*}\left\langle\hat{\mathrm{A}}^{2} d, g \times d\right\rangle_{\mathrm{H}^{1}} & =(\nabla \Delta d, \nabla(g \times d)) \\
& \leq C|\nabla \Delta d|_{L^{2}}\left(|\nabla g|_{L^{2}}+|g|_{L^{4}}|\nabla d|_{L^{4}}\right) \\
& \leq C|\nabla \Delta d|_{L^{2}}\left(|\nabla g|_{L^{2}}+|g|_{L^{4}}|\nabla d|_{L^{2}}^{\frac{1}{2}}|\Delta d|_{L^{2}}^{\frac{1}{2}}\right) \\
& \leq \frac{1}{2}|\nabla \Delta d|_{L^{2}}^{2}+\frac{1}{2}|g|_{\mathrm{H}^{1}}^{2}+C|g|_{\mathrm{H}^{1}}^{2}\left(|\nabla d|_{L^{2}}^{2}+|\Delta d|_{L^{2}}^{2}\right) .
\end{aligned}
$$

Plugging these estimates and the ones in Claims 4.10-4.11 into (4.23) yield

$$
\begin{aligned}
& \frac{1}{2} \frac{\mathrm{d}}{\mathrm{d} t}\left(|\Delta d|_{L^{2}}^{2}+|\nabla v|_{L^{2}}^{2}\right)+\frac{1}{2}\left(|\mathrm{~A} v|_{L^{2}}^{2}+|\nabla \Delta d|_{L^{2}}^{2}\right)-C|v|_{L^{4}}^{4}|\nabla v|_{L^{2}}^{2} \\
& \leq C\left(1+|\nabla d|_{L^{4}}^{4}+|g|_{\mathrm{H}^{1}}^{2}\right)|\Delta d|_{L^{2}}^{2}+\frac{1}{2}\left(|f|_{L^{2}}^{2}+|g|_{\mathrm{H}^{1}}^{2}+C|g|_{\mathrm{H}^{1}}^{2}|\nabla d|_{L^{2}}^{2}\right) \\
& \quad+C|\nabla d|^{2}|\nabla d|_{L^{4}}^{4} .
\end{aligned}
$$

Hence,

$$
\begin{aligned}
& \frac{1}{2} \frac{\mathrm{d}}{\mathrm{d} t}\left(|\Delta d(t)|_{L^{2}}^{2}+|\nabla v(t)|_{L^{2}}^{2}\right) \\
& \leq C\left(1+|\nabla d(t)|_{L^{4}}^{4}+|g(t)|_{\mathrm{H}^{1}}^{2}+|v(t)|_{L^{4}}^{4}\right)\left(|\Delta d(t)|_{L^{2}}^{2}+|\nabla v(t)|_{L^{2}}^{2}\right) \\
& \quad+\frac{1}{2}\left(|f(t)|_{L^{2}}^{2}+|g(t)|_{\mathrm{H}^{1}}^{2}+C|g(t)|_{\mathrm{H}^{1}}^{2}|\nabla d(t)|_{L^{2}}^{2}\right)+C|\nabla d(t)|^{2}|\nabla d(t)|_{L^{4}}^{4} .
\end{aligned}
$$


Let us put

$$
\Theta(t):=e^{2 C\left(\int_{0}^{t}\left[|\nabla d(r)|_{L^{4}}^{4}+|\nabla d(r)|_{L^{4}}^{4}+|g(r)|_{\mathrm{H}^{1}}^{2}+|v(r)|_{L^{4}}^{2}+|v(r)|_{L^{4}}^{4}\right] d r\right)} \mathrm{d} s, t \in\left[0, T_{*}\right) .
$$

Thus, by the Gronwall lemma, we obtain

$$
\begin{aligned}
& |\Delta d(t)|_{L^{2}}^{2}+|\nabla u(t)|_{L^{2}}^{2}-\left(\left|\Delta d_{0}\right|_{L^{2}}^{2}+\left|\nabla v_{0}\right|_{L^{2}}^{2}\right) \Theta(t) \\
& \quad \leq\left(\int_{0}^{t} \frac{1}{2}|f(s)|_{L^{2}}^{2} \mathrm{~d} s+\left(C \sup _{s \in[0, \tau]}|\nabla d(s)|_{L^{2}}^{2}+\frac{1}{2}\right) \int_{0}^{t}\left[|g(s)|_{\mathrm{H}^{1}}^{2}+|\nabla d(s)|_{L^{4}}^{4}\right] \mathrm{d} s\right) \Theta(t),
\end{aligned}
$$

which along with the inequality (4.9) the fact $\theta \leq e^{\theta}, \theta \geq 0$ implies that

$$
\begin{aligned}
& \sup _{0 \leq s \leq \tau}\left(|\nabla v(s)|_{L^{2}}^{2}+|\Delta d(s)|_{L^{2}}^{2}\right) \\
& \leq C \Sigma_{0} e^{C \Xi(T)} e^{C \int_{0}^{\tau}\left[|\nabla d(r)|_{L^{4}}^{4}+|v(r)|_{L^{4}}^{4}+|\nabla d(r)|_{L^{4}}^{2}+|v(r)|_{L^{4}}^{2}\right] d r .}
\end{aligned}
$$

Integrating (4.24), using (4.27) and the fact $\theta \leq e^{\theta}, \theta \geq 0$ yield

$$
\begin{aligned}
\int_{0}^{\tau}\left(|\mathrm{A} v(s)|_{L^{2}}^{2}+|\nabla \Delta d(r)|^{2}\right) \mathrm{d} s \leq & \sup _{s \in[0, \tau]}|\Delta d(s)|_{L^{2}}^{2} C \int_{0}^{\tau}|\nabla d(s)|_{L^{4}}^{4}+|g(s)|_{\mathrm{H}^{1}}^{2} \mathrm{~d} s \\
& +\int_{0}^{\tau}\left(|f(s)|_{L^{2}}^{2}+|g(s)|_{\mathrm{H}^{1}}^{2}\right) \mathrm{d} s+\left|\nabla v_{0}\right|_{L^{2}}^{2}+\left|\Delta d_{0}\right|_{L^{2}}^{2} \\
& +C \sup _{s \in[0, \tau)}|\nabla d(s)|_{L^{2}}^{2} \int_{0}^{\tau}|g(s)|_{\mathrm{H}^{1}}^{2} \mathrm{~d} s \\
\leq & C \Sigma_{0} e^{C \Xi(T)} e^{C \int_{0}^{\tau}\left[|\nabla d(r)|_{L^{4}}^{4}+|v(r)|_{L^{4}}^{4}+|\nabla d(r)|_{L^{4}}^{2}+|v(r)|_{L^{4}}^{2}\right] d r}
\end{aligned}
$$

We easily infer from (4.27) and (4.28) that (4.22) holds. This completes the proof of the lemma.

We have the following consequence of the above lemma.

Corollary 4.13. Let $\left(v_{0}, d_{0}\right) \in \mathrm{V} \times D(\hat{\mathrm{A}}),(f, g) \in L^{2}\left(0, T ; L^{2} \times \mathrm{H}^{1}\right), r_{0}$ and $\varepsilon_{0}$ be as in Lemmas 4.1 and 4.9. Let $(v, d) \in X_{T_{*}}$ be a maximal local regular solution to the problem (2.13). Let $\Sigma_{0}$ be defined as in (4.21).

Then, there exists a constant $K_{3}>0$ such that for any $R \in\left(0, r_{0}\right], \varepsilon \in\left(0, \varepsilon_{0}\right)$ and $t \in[0, T(\varepsilon, R)]$,

$$
\begin{aligned}
& \left(|\nabla v(t)|_{L^{2}}^{2}+|\Delta d(t)|_{L^{2}}^{2}\right)+2 \int_{0}^{t}\left(|\nabla \Delta d(s)|_{L^{2}}^{2}+|\mathrm{A} v(s)|_{L^{2}}^{2}\right) \mathrm{d} s \\
& \quad \leq K_{3} \Sigma_{0} e^{K_{3}\left[\Xi(T)+\varepsilon^{2}\left(E_{0}+\Psi(T)+\left(1+\frac{2 \varepsilon^{2}}{R^{2}}\right) t\right)\right]} .
\end{aligned}
$$

Proof. Let $(v, d) \in X_{T_{*}}$ be a maximal local regular solution to the problem (2.13) with initial data $\left(v_{0}, d_{0}\right) \in \mathrm{V} \times D(\hat{\mathrm{A}})$. Let us fix $R \in\left(0, r_{0}\right]$ and $\varepsilon \in\left(0, \varepsilon_{0}\right)$. Since $(v, d) \in X_{T(\varepsilon, R)}$, we can apply Lemma 4.12 and infer that (4.22) holds for 
$\tau=T(\varepsilon, R)$. Hence, in order to complete the proof of the corollary we need to estimate the exponential term in the right-hand side of (4.22). For this purpose, we use (4.13) and infer that there exists a universal constant $K>0$ such that for any $R \in\left(0, r_{0}\right], \varepsilon\left(0, \varepsilon_{0}\right)$ and $t \in[0, T(\varepsilon, R)]$

$$
e^{K_{4} \int_{0}^{t}\left(|\nabla d(s)|_{L^{4}}^{4}+|v(s)|_{L^{4}}^{4}+|\nabla d(s)|_{L^{4}}^{2}+|v(s)|_{L^{4}}^{2}\right) \mathrm{d} s} \leq K e^{K\left[\Xi(T)+\varepsilon^{2}\left(E_{0}+\Psi(T)+\left(1+\frac{2 \varepsilon^{2}}{R^{2}}\right) t\right)\right]} .
$$

This completes the proof of the corollary.

Corollary 4.14. Let $\left(v_{0}, d_{0}\right) \in \mathrm{V} \times D(\hat{\mathrm{A}}),(f, g) \in L^{2}\left(0, T ; L^{2} \times \mathrm{H}^{1}\right)$ and $(v, d) \in$ $X_{T_{*}}$ be a maximal local regular solution to the problem (2.13). Let $r_{0}>0$ and $\varepsilon_{0}>0$ be as in Lemmas 4.1 and 4.9, respectively. Then, for all $\varepsilon \in\left(0, \varepsilon_{0}\right)$ and $R \in\left(0, r_{0}\right]$ we have

$$
T(\varepsilon, R)<T_{*}
$$

Proof. We argue by contradiction. Assume that there exists $\varepsilon \in\left(0, \varepsilon_{0}\right)$ and $R \in$ $\left(0, r_{0}\right]$ such that $T(\varepsilon, R)=T_{*}$. Let us put

$$
R_{2}=\int_{0}^{T_{*}}\left(|f(r)|_{L^{2}}^{2}+|g(r)|_{\mathrm{H}^{1}}^{2}\right) d r .
$$

By Corollary 4.13 and (4.9) we infer that there exists a constant $\tilde{K}_{3}>0$ such that for all $t \in\left[0, T_{*}\right)$

$$
\mid\left(v(t),\left.d(t)\right|_{\mathrm{V} \times D(\hat{\mathrm{A}})} ^{2} \leq \tilde{K}_{3} .\right.
$$

Let $T_{0}=T_{1}\left(\tilde{K}_{3}, R_{2}\right) \wedge T_{2}(g) \wedge T_{*}>0$ be the time given by Theorem 3.7. Let $T_{1}=\frac{T_{0}}{2}$. By Theorem 3.7 the problem (2.13) with initial data $\left(v\left(T_{1}\right), d\left(T_{1}\right)\right)$ has a unique local regular solution $(\tilde{v}(t), \tilde{d}(t))$ defined on $\left[T_{*}-T_{1}, T_{*}-T_{1}+T_{0}\right]$. We then define $(\bar{v}, \bar{d}):\left[0, T_{*}+T_{1}\right] \rightarrow \mathrm{V} \times D(\hat{\mathrm{A}})$ by

$$
(\bar{v}(t), \bar{d}(t))=\left\{\begin{array}{l}
(v(t), d(t)) \text { if } t \in\left[0, T_{*}-T_{1}\right] \\
(\tilde{v}(t), \tilde{d}(t)) \text { if } t \in\left[T_{*}-T_{1}, T_{*}+T_{1}\right] .
\end{array}\right.
$$

It is easily seen that $\left((\bar{v}, \bar{d}) ; T_{1}\right)$ is a local regular solution to (2.13) with initial data $\left(v_{0}, d_{0}\right)$ and time of existence $T_{*}+T_{1}>T_{*}$. This contradicts the fact that $\left((v, d) ; T_{*}\right)$, with $T_{*}=T(\varepsilon, R)$, is a maximal smooth solution to (2.13). This completes the proof of the corollary.

We now state and prove a local energy inequality which will play an important role in the proof of Proposition 4.3.

Lemma 4.15. Let $\left(v_{0}, d_{0}\right) \in \mathrm{V} \times D(\hat{\mathrm{A}}),(f, g) \in L^{2}\left(0, T ; L^{2} \times \mathrm{H}^{1}\right), r_{0}$ and $\varepsilon_{0}$ be as in Lemma 4.9. Also, let $R \in\left(0, r_{0}\right], \varepsilon \in\left(0, \varepsilon_{0}\right)$ and $(v, d) \in X_{T_{*}}$ be a local regular 
solution to the problem (2.13). Then, there exists a function $\mathrm{p}:\left[0, T_{*}\right) \rightarrow L^{1}$ such that $\mathrm{p} \in L^{\frac{4}{3}}\left(0, T_{*} ; L^{4}\right), \nabla \mathrm{p} \in L^{\frac{4}{3}}\left(0, T_{*} ; L^{\frac{4}{3}}\right)$ and

$$
\begin{aligned}
& v(t)+\int_{0}^{t}[v(s) \cdot \nabla v(s)+\nabla \mathrm{p}(s)] \mathrm{d} s=v_{0} \\
& +\int_{0}^{t}[\Delta v(s)-\nabla d(s) \Delta d(s)+f(s)] \mathrm{d} s, t \in\left[0, T_{*}\right] .
\end{aligned}
$$

Moreover, there exists a constant $K_{5}>0$, which may depend on the norms of $\left(v_{0}, d_{0}\right) \in$ $\mathrm{V} \times D(\hat{\mathrm{A}})$ and $(f, g) \in L^{2}\left(0, T ; L^{2} \times \mathrm{H}^{1}\right)$, such that for any $t \in\left[0, T_{*}\right)$

$$
|\nabla \mathrm{p}|_{L^{\frac{4}{3}}\left(0, t ; L^{\frac{4}{3}}\right)} \leq K_{5} \varepsilon_{1}^{\frac{1}{2}}\left[E_{0}+\Psi(t)+\left(1+\frac{2 \varepsilon^{2}}{R^{2}}\right) t\right]^{\frac{3}{4}} .
$$

Proof. Let us fix $\varepsilon \in\left(0, \varepsilon_{0}\right)$ and $R \in\left(0, r_{0}\right]$. We fix $t \in\left[0, T_{*}\right)$. From (4.9) and (4.12) we have $(v, d) \in C\left([0, t] ; \mathrm{H} \times \mathrm{H}^{1}\right) \cap L^{2}(0, t ; \mathrm{V} \times D(\hat{\mathrm{A}}))$. Hence, one can apply [26, Lemma 4.4] and infer that there exists function $\mathrm{p}:\left[0, T_{*}\right) \rightarrow L^{1}$ such that $\mathrm{p} \in L^{\frac{4}{3}}\left(0, T_{*} ; L^{4}\right), \nabla \mathrm{p} \in L^{\frac{4}{3}}\left(0, T_{*} ; L^{\frac{4}{3}}\right)$ and the identity (4.31) holds. Moreover,

$$
|\nabla|_{L^{\frac{4}{3}}\left(0, t ; L^{\frac{4}{3}}\right)} \leq|f|_{L^{\frac{4}{3}}\left(0, t ; L^{\frac{4}{3}}\right)}+|v \cdot \nabla v|_{L^{\frac{4}{3}}\left(0, t ; L^{\frac{4}{3}}\right)}+|\nabla d \Delta d|_{L^{\frac{4}{3}}\left(0, t ; L^{\frac{4}{3}}\right)} .
$$

From the Hölder inequality, (4.9) and (4.13) we infer that

$$
\begin{aligned}
|v \cdot \nabla v|_{L^{\frac{4}{3}}\left(0, t ; L^{\frac{4}{3}}\right)} & \leq\left(\int_{0}^{t}|v(r)|_{L^{4}}^{4} d r\right)^{\frac{1}{4}}\left(\int_{0}^{t}|\nabla v(r)|_{L^{2}}^{2} d r\right)^{\frac{1}{2}} \\
& \leq C \varepsilon^{\frac{1}{2}}\left[E_{0}+\Psi(t)+\left(1+\frac{2 \varepsilon^{2}}{R^{2}}\right) t\right]^{\frac{3}{4}} .
\end{aligned}
$$

In a similar way,

$$
\begin{aligned}
|\nabla d \Delta d|_{L^{\frac{4}{3}}\left(0, t ; L^{\frac{4}{3}}\right)} & \leq\left(\int_{0}^{t}|\nabla d(r)|_{L^{4}}^{4} d r\right)^{\frac{1}{4}}\left(\int_{0}^{t}|\Delta d(r)|_{L^{2}}^{2} d r\right)^{\frac{1}{2}} \\
& \leq C \varepsilon^{\frac{1}{2}}\left[E_{0}+\Psi(t)+\left(1+\frac{2 \varepsilon^{2}}{R^{2}}\right) t\right]^{\frac{3}{4}} .
\end{aligned}
$$

Plugging (4.33) and (4.34) into (4.32) completes the proof of Lemma 4.15.

We now continue with some estimates of local energy.

Lemma 4.16. Let $\left(v_{0}, d_{0}\right) \in \mathrm{V} \times D(\hat{\mathrm{A}}),(f, g) \in L^{2}\left(0, T ; L^{2} \times \mathrm{H}^{1}\right)$ and $(v, d) \in X_{T_{*}}$ be a maximal local regular solution to the problem (2.13).

Let $\varphi \in C_{c}^{\infty}(\Omega ; \mathbb{R})$ and put

$$
\mathcal{E}_{\varphi}(v(t), d(t))=\frac{1}{2} \int_{\Omega} \varphi(x)\left(|v(t, x)|^{2}+|\nabla d(t, x)|^{2}+2 \phi(d(t, x))\right) \mathrm{d} x, \quad t \in\left[0, T_{*}\right) .
$$


We also set

$$
\mathrm{p}_{\Omega}(t)=\frac{1}{|\Omega|} \int_{\Omega} \mathrm{p}(t, x) \mathrm{d} x, t \in\left[0, T_{*}\right) .
$$

Then, for any $s, t \in\left[0, T_{*}\right)$ with $s \leq t$ we have

$$
\begin{aligned}
& \mathcal{E}_{\varphi}(v(t), d(t))-\mathcal{E}_{\varphi}(v(s), d(s))+\int_{s}^{t} \int_{\Omega} \varphi\left(|\nabla v(r)|^{2}+|R(d(r))|^{2}\right) \mathrm{d} x \mathrm{~d} r \\
& \leq \int_{s}^{t} \int_{\Omega}|\nabla \varphi|\left(\frac{1}{2}|\nabla d(r)|^{2}|v(r)|+\left|\partial_{t} d(r)\right||\nabla d(r)|+\phi(d(r))|v(r)|+|v(r)|^{3}\right) \mathrm{d} x \mathrm{~d} r \\
& \quad+\int_{s}^{t} \int_{\Omega}|\nabla \varphi|\left(|\nabla v(r)||v(r)|+\left|\mathrm{p}(r)-\mathrm{p}_{\Omega}(r)\right||v(r)|+|g(r)||\nabla d(r)|\right) \mathrm{d} x \mathrm{~d} r \\
& \quad+\int_{s}^{t} \int_{\Omega}|\varphi|\left(|d(r)||\nabla g(r)||\nabla d(r)|+|d(r)||g(r)|\left|\phi^{\prime}(d(r))\right|+|f(r)||v(r)|\right) \mathrm{d} x \mathrm{~d} r .
\end{aligned}
$$

Proof. Throughout this proof, in order to save space we simply write $\int_{\mathcal{O}} \Xi d \mu$ instead of $\int_{\mathcal{O}} \Xi(y) d \mu(y)$ for an integrable function $\Xi$ defined on a measure space $(\mathcal{O}, \mathcal{A}, \mu)$.

We fix $\varphi \in C_{c}^{\infty}(\Omega ; \mathbb{R})$ and $s \leq t \in\left[0, T_{*}\right)$. We firstly observe that because of the fact $d(t) \in \mathcal{M}$ for all $t \in\left[0, T_{*}\right)$ we have

$$
\left(\partial_{t} d+v \cdot \nabla d\right) \cdot\left(|\nabla d|^{2} d+\alpha(d) d\right)=0
$$

for all $t \in\left[0, T_{*}\right)$ and a.e. $x \in \Omega$. Multiplying $\partial_{t} d+v \cdot \nabla d$ by $-\varphi R(d)$ in $L^{2}$ and using the pointwise orthogonality yields

$$
\begin{aligned}
A+B & :=-\int_{\Omega} \varphi\left(\partial_{t} d+v \cdot \nabla d\right) \cdot \Delta d \mathrm{~d} x+\int_{\Omega}\left(\partial_{t} d+v \cdot \nabla d\right) \cdot \phi^{\prime}(d) \mathrm{d} x \\
& =-\int_{\Omega} \varphi|R(d)|^{2} \mathrm{~d} x-\int_{\Omega} \varphi(d \times g) \cdot R(d) \mathrm{d} x .
\end{aligned}
$$

Using integration by parts and [26, Eq. (4.16)] we obtain

$$
\begin{aligned}
A= & \frac{1}{2} \frac{\mathrm{d}}{\mathrm{d} t} \int_{\Omega}|\nabla d|^{2} \varphi \mathrm{d} x+\int_{\Omega} \partial_{t} d \cdot(\nabla d \nabla \varphi) \mathrm{d} x-\int_{\Omega} \varphi(v \cdot \nabla d) \cdot \Delta d \mathrm{~d} x \\
= & \frac{1}{2} \frac{\mathrm{d}}{\mathrm{d} t} \int_{\Omega}|\nabla d|^{2} \varphi \mathrm{d} x+\int_{\Omega} \partial_{t} d \cdot(\nabla d \nabla \varphi) \mathrm{d} x-\int_{\Omega} \frac{1}{2}|\nabla d|^{2} v \cdot \nabla \varphi \mathrm{d} x \\
& +\int_{\Omega} \varphi(\nabla d \odot \nabla d) \nabla u \mathrm{~d} x \\
& -\int_{\Omega}(v \cdot \nabla d) \cdot(\nabla d \nabla \varphi) \mathrm{d} x .
\end{aligned}
$$

For the term $B$ it is easy to show that

$$
B=\int_{\Omega}\left(\partial_{t} d \cdot \phi^{\prime}(d)\right) \varphi \mathrm{d} x+\int_{\Omega} \varphi(v \cdot \nabla d) \cdot \phi^{\prime}(d) \mathrm{d} x
$$




$$
\begin{aligned}
& =\frac{\mathrm{d}}{\mathrm{d} t} \int_{\Omega} \phi(d) \varphi \mathrm{d} x+\int_{\Omega} v \cdot \nabla \phi(d) \varphi \mathrm{d} x \\
& =\frac{\mathrm{d}}{\mathrm{d} t} \int_{\Omega} \phi(d) \varphi \mathrm{d} x-\int_{\Omega} v \cdot \nabla \varphi \phi(d) \mathrm{d} x .
\end{aligned}
$$

Note also that for all $t \in\left[0, T_{*}\right)$ and a.e. $x \in \Omega$.

$$
(d \times g) \cdot\left(|\nabla d|^{2} d+\alpha(d) d\right)=0 .
$$

Hence, using integration by parts and the Cauchy-Schwarz inequality we obtain

$$
\begin{aligned}
-\int_{\Omega} \varphi(d \times g) \cdot R(d) \mathrm{d} x= & -\int_{\Omega} \varphi(d \times g) \cdot\left(\Delta d-\phi^{\prime}(d)\right) \mathrm{d} x \\
\leq & \int_{\Omega}|\varphi|(|d||\nabla d| \nabla g \mid) \mathrm{d} x+\int_{\Omega}|g| \nabla d|| \nabla \varphi \mid \mathrm{d} x \\
& +\int_{\Omega}|g||d|\left|\phi^{\prime}(d)\right||\varphi| \mathrm{d} x
\end{aligned}
$$

Plugging (4.38), (4.39) and (4.40) into (4.37) yields

$$
\begin{aligned}
\frac{1}{2} & \frac{\mathrm{d}}{\mathrm{d} t} \int_{\Omega}\left(|\nabla d|^{2}+2 \phi(d)\right) \varphi \mathrm{d} x+\int_{\Omega} \varphi|R(d)|^{2} \mathrm{~d} x \\
\leq & \int_{\Omega} \frac{1}{2}|\nabla d|^{2} v \cdot \nabla \varphi \mathrm{d} x+\int_{\Omega}(v \cdot \nabla d) \cdot(\nabla d \nabla \varphi) \mathrm{d} x-\int_{\Omega} \partial_{t} d \cdot(\nabla d \nabla \varphi) \mathrm{d} x \\
& -\int_{\Omega} \varphi(\nabla d \odot \nabla d) \nabla u \mathrm{~d} x+\int_{\Omega}|\varphi|(|d||\nabla d| \nabla g \mid) \mathrm{d} x+\int_{\Omega}|g| \nabla d|| \nabla \varphi \mid \mathrm{d} x \\
& +\int_{\Omega}|g||d|\left|\phi^{\prime}(d)\right||\varphi| \mathrm{d} x .
\end{aligned}
$$

We can follow the same calculation in [26] to derive the following local inequality for the velocity $v$

$$
\begin{aligned}
& \frac{1}{2} \frac{\mathrm{d}}{\mathrm{d} t} \int_{\Omega}|v|^{2} \varphi \mathrm{d} x+\int_{\Omega} \leq \frac{1}{4} \int_{\Omega}|v|^{2} v \cdot \nabla \varphi \mathrm{d} x-\int_{\Omega}(\nabla u) v \cdot \nabla \varphi \mathrm{d} x+\int_{\Omega}\left(\mathrm{p}-\mathrm{p}_{\Omega}\right) v \cdot \nabla \varphi \mathrm{d} x \\
& \quad+\int_{\Omega} \varphi(\nabla d \odot \nabla d) \nabla u \mathrm{~d} x+\int_{\Omega}(\nabla d) \cdot \nabla d \nabla \varphi \mathrm{d} x+\int_{\Omega}|f||v||\varphi| \mathrm{d} x .
\end{aligned}
$$

Adding up the last inequalities side by side and using the Cauchy-Schwarz inequality and integrating over $[s, t]$ yield the sought estimate (4.35).

The following lemma is also important for our analysis.

Lemma 4.17. Let $\left(v_{0}, d_{0}\right) \in \mathrm{V} \times D(\hat{\mathrm{A}}),(f, g) \in L^{2}\left(0, T ; L^{2} \times \mathrm{H}^{1}\right), r_{0}$ and $\varepsilon_{0}$ be as in Lemma 4.9. Also, let $(v, d) \in X_{T_{*}}$ be a maximal local regular solution to the problem (2.13).

Then, there exists a constant $K_{4}>0$ such that for all $\varepsilon \in\left(0, \varepsilon_{0}\right), R \in\left(0, r_{0}\right]$ and $t \in[0, T(\varepsilon, R)]$ 


$$
\begin{aligned}
& \frac{1}{2} \int_{B(x, R)}\left(|v(t)|^{2}+|\nabla d(t)|^{2}+2 \phi(d(t))\right) \mathrm{d} y-\frac{1}{2} \int_{B(x, 2 R)}\left(\left|v_{0}\right|^{2}+\left|\nabla d_{0}\right|^{2}+2 \phi\left(d_{0}\right)\right) \mathrm{d} y \\
& \leq K_{4} t^{\frac{1}{4}}\left(1+E_{0}+\frac{1}{2} \int_{0}^{t}\left[|f(s)|_{\mathrm{H}^{-1}}^{2}+|g(s)|_{L^{2}}^{2}\right] \mathrm{d} s+\left(1+\frac{2 \varepsilon^{2}}{R^{2}}\right) t\right)^{\frac{5}{4}}\left(R^{-\frac{1}{2}}\left(\varepsilon^{\frac{3}{2}}+\varepsilon^{\frac{1}{2}}\right)+\varepsilon^{\frac{1}{2}}\right) .
\end{aligned}
$$

Proof. Throughout this proof, in order to save space we simply write $\int_{\mathcal{O}} \Xi d \mu$ instead of $\int_{\mathcal{O}} \Xi(y) d \mu(y)$ for an integrable function $\Xi$ defined on a measure space $(\mathcal{O}, \mathcal{A}, \mu)$.

Let us fix $\varepsilon \in\left(0, \varepsilon_{0}\right), R \in\left(0, r_{0}\right], x \in \Omega$ and $t \in[0, T(\varepsilon, R)]$. We also fix $\varphi \in C_{c}^{\infty}(\Omega ;[0,1])$ such that

$$
\mathbb{1}_{B(x, R)} \leq \varphi \leq \mathbb{1}_{B(x, 2 R)} \text { and }|\nabla \varphi| \leq \frac{c_{4}}{R}
$$

for some constant $c_{4}>0$. For such particular $\varphi$ we will estimate each term on the right hand side of (4.35). To start this quest we observe that

$$
\left(\int_{0}^{t} \int_{B(x, 2 R)}|\nabla \varphi| \mathrm{d} x \mathrm{~d} s\right)^{\frac{1}{4}} \leq \frac{c_{4}}{R}|B(x, 2 R)|^{\frac{1}{4}} t^{\frac{1}{4}} \leq c_{5}\left(\frac{t}{R^{2}}\right)^{\frac{1}{4}} .
$$

We will use this inequality below without further notice.

Using the Hölder inequality and (4.13) we get

$$
\begin{aligned}
\int_{0}^{t} \int_{\Omega} \frac{|\nabla d|^{2}}{2}|v||\nabla \varphi| \mathrm{d} x \mathrm{~d} r & \leq\left(\int_{0}^{t}|\nabla d|_{L^{4}}^{4}\right)^{\frac{1}{4}}\left(\int_{0}^{t}|v|_{L^{4}}^{4}\right)^{\frac{1}{4}}\left(\int_{0}^{t}|\nabla \varphi|_{L^{4}}^{4}\right)^{\frac{1}{4}} \\
& \leq C \varepsilon^{\frac{3}{2}}\left(\frac{t}{R^{2}}\right)^{\frac{1}{4}}\left[E_{0}+\Psi(t)+\left(1+\frac{2 \varepsilon^{2}}{R^{2}}\right) t\right]^{\frac{3}{4}}
\end{aligned}
$$

In a similar way we get

$$
\int_{0}^{t} \int_{\Omega}|\nabla \varphi| \phi(d)|v| \mathrm{d} x \mathrm{~d} r \leq C \varepsilon^{\frac{1}{2}}\left(\frac{t}{R^{2}}\right)^{\frac{1}{4}}\left[E_{0}+\Psi(t)+\left(1+\frac{2 \varepsilon^{2}}{R^{2}}\right) t\right]^{\frac{3}{4}} .
$$

Here, we used the boundedness of $\phi$ on $\mathbb{S}^{2}$. Similarly,

$$
\begin{aligned}
& \int_{0}^{t} \int_{\Omega}|\nabla \varphi||v|^{3} \mathrm{~d} x \mathrm{~d} r \leq C \varepsilon^{\frac{3}{2}}\left(\frac{t}{R^{2}}\right)^{\frac{1}{4}}\left[E_{0}+\Psi(t)+\left(1+\frac{2 \varepsilon^{2}}{R^{2}}\right) t\right]^{\frac{3}{4}} \\
& \int_{0}^{t} \int_{\Omega}|\nabla \varphi||g||\nabla d| \mathrm{d} x \mathrm{~d} r \leq C \varepsilon^{\frac{1}{2}}\left(\frac{t}{R^{2}}\right)^{\frac{1}{4}}\left[E_{0}+\Psi(t)+\left(1+\frac{2 \varepsilon^{2}}{R^{2}}\right) t\right]^{\frac{3}{4}} .
\end{aligned}
$$

Using the Hölder inequality, (4.13) and (4.9) 


$$
\begin{aligned}
\int_{0}^{t} \int_{\Omega}|\nabla \varphi||\nabla v \| v| \mathrm{d} x \mathrm{~d} r & \leq c_{5}\left(\frac{t}{R^{2}}\right)^{\frac{1}{4}}\left(\int_{0}^{t}|v|_{L^{4}}^{4} d r\right)^{\frac{1}{4}}\left(\int_{0}^{t}|\nabla v|_{L^{2}}^{2} d r\right)^{\frac{1}{2}} \\
& \leq C \varepsilon^{\frac{1}{2}}\left(\frac{t}{R^{2}}\right)^{\frac{1}{4}}\left[E_{0}+\Psi(t)+\left(1+\frac{2 \varepsilon^{2}}{R^{2}}\right) t\right]^{\frac{3}{4}} .
\end{aligned}
$$

To deal with the term containing $\partial_{t} d$ we argue as follows:

$$
\begin{aligned}
& \int_{0}^{t} \int_{\Omega}\left|\partial_{t} d \| \nabla d\right||\nabla \varphi| \mathrm{d} x \mathrm{~d} r \leq c_{5}\left(\frac{t}{R^{2}}\right)^{\frac{1}{4}}\left(\int_{0}^{t}|\nabla d|_{L^{4}}^{4} d r\right)^{\frac{1}{4}}\left(\int_{0}^{t}\left|\partial_{t} d\right|_{L^{2}}^{2} d r\right)^{\frac{1}{2}} \\
& \quad \leq c_{5}\left(\frac{t}{R^{2}}\right)^{\frac{1}{4}}\left(\int_{0}^{t}|\nabla d|_{L^{4}}^{4} d r\right)^{\frac{1}{4}}\left(\int_{0}^{t}\left[|R(d)|_{L^{2}}^{2}+|g|_{L^{2}}^{2}\right] d r\right)^{\frac{1}{2}} .
\end{aligned}
$$

Now, using (4.13) and (4.9) we obtain

$$
\int_{0}^{t} \int_{\Omega}\left|\partial_{t} d\right||\nabla d||\nabla \varphi| \mathrm{d} x \mathrm{~d} r \leq C \varepsilon^{\frac{1}{2}}\left(\frac{t}{R^{2}}\right)^{\frac{1}{4}}\left[E_{0}+\Psi(t)+\left(1+\frac{2 \varepsilon^{2}}{R^{2}}\right) t\right]^{\frac{3}{4}} .
$$

We now deal with term containing the pressure p. First by the Using the Hölder and Poincaré inequalities and the estimates (4.9) we obtain

$$
\begin{aligned}
\int_{0}^{t} \int_{\Omega}\left|\mathrm{p}-\mathrm{p}_{\Omega}\right||v||\nabla \varphi| \mathrm{d} x \mathrm{~d} r & \leq \sup _{0 \leq t<T_{*}}|v(t)|_{L^{2}}\left(\int_{0}^{t}\left|\mathrm{p}-\mathrm{p}_{\Omega}\right|_{L^{4}}^{\frac{4}{2}} d r\right)^{\frac{3}{4}}\left(\int_{0}^{t}|\nabla \varphi|_{L^{4}}^{4}\right)^{\frac{1}{4}} \\
& \leq C\left(\frac{t}{R^{2}}\right)^{\frac{1}{4}}\left[E_{0}+\Psi(t)\right]^{\frac{1}{2}}\left(\int_{0}^{t}\left|\mathrm{p}-\mathrm{p}_{\Omega}\right|_{L^{4}}^{\frac{4}{3}} d r\right)^{\frac{3}{4}} \\
& \leq C\left(\frac{t}{R^{2}}\right)^{\frac{1}{4}}\left[E_{0}+\Psi(t)\right]^{\frac{1}{2}}\left(\int_{0}^{t}|\nabla \mathrm{p}|_{L^{\frac{4}{3}}}^{\frac{4}{3}} d r\right)^{\frac{3}{4}} .
\end{aligned}
$$

From the last line and Lemma 4.15 we infer that

$$
\int_{0}^{t} \int_{\Omega}\left|\mathrm{p}-\mathrm{p}_{\Omega}\right||v||\nabla \varphi| \mathrm{d} x \mathrm{~d} r \leq C \varepsilon^{\frac{1}{2}}\left(\frac{t}{R^{2}}\right)^{\frac{1}{4}}\left[E_{0}+\Psi(t)+\left(1+\frac{2 \varepsilon^{2}}{R^{2}}\right) t\right]^{\frac{5}{4}} .
$$

We now deal with the terms containing $|\varphi|$. Applying the Hölder inequality and (4.13) yields

$$
\begin{aligned}
\int_{0}^{t} \int_{\Omega}|\varphi||\nabla g \| \nabla d| \mathrm{d} x \mathrm{~d} r & \leq C\left(\int_{0}^{t} \int_{\Omega} \varphi^{4} \mathrm{~d} x \mathrm{~d} r\right)^{\frac{1}{4}}\left(\int_{0}^{t} \int_{\Omega}|\nabla g|^{2} \mathrm{~d} x \mathrm{~d} r\right)^{\frac{1}{2}}\left(\int_{0}^{t} \int_{\Omega}|\nabla d|^{4} \mathrm{~d} x \mathrm{~d} r\right)^{\frac{1}{4}} \\
& \leq C t^{\frac{1}{4}} \varepsilon^{\frac{1}{2}}\left[E_{0}+\Psi(t)+\left(1+\frac{2 \varepsilon^{2}}{R^{2}}\right) t\right]^{\frac{3}{4}} .
\end{aligned}
$$


In a similar way,

$$
\begin{aligned}
& \int_{0}^{t} \int_{\Omega}|\varphi||g|\left|\phi^{\prime}(d)\right| \mathrm{d} x \mathrm{~d} r \leq C\left(\int_{0}^{t} \int_{\Omega} \varphi^{4} \mathrm{~d} x \mathrm{~d} r\right)^{\frac{1}{4}}\left(\int_{0}^{t} \int_{\Omega}|g|^{2} \mathrm{~d} x \mathrm{~d} r\right)^{\frac{1}{2}}\left(\int_{0}^{t} \int_{\Omega}|d|^{4} \mathrm{~d} x \mathrm{~d} r\right)^{\frac{1}{4}} \\
& \quad \leq C\left(\int_{0}^{t} \int_{\Omega} \varphi^{4} \mathrm{~d} x \mathrm{~d} r\right)^{\frac{1}{4}}\left(\int_{0}^{t} \int_{\Omega}|g|^{2} \mathrm{~d} x \mathrm{~d} r\right)^{\frac{1}{2}}\left(1+\sup _{r \in[0, t]}|\nabla d(r)|_{L^{2}}\right) \\
& \leq C t^{\frac{1}{4}} \varepsilon^{\frac{1}{2}}\left[1+E_{0}+\Psi(t)+\left(1+\frac{2 \varepsilon^{2}}{R^{2}}\right) t\right] .
\end{aligned}
$$

We also have

$$
\int_{0}^{t} \int_{\Omega}|f||v \| \varphi| \mathrm{d} x \mathrm{~d} r \leq C t^{\frac{1}{4}} \varepsilon^{\frac{1}{2}}\left[E_{0}+\Psi(t)+\left(1+\frac{2 \varepsilon^{2}}{R^{2}}\right) t\right]^{\frac{3}{4}} .
$$

Taking $s=0$, dropping out the positive term $\int_{0}^{t} \int_{\Omega} \varphi|R(d)|^{2} \mathrm{~d} x \mathrm{~d} r$, plugging the inequalities (4.44)-(4.45) and using the first fact in (4.43) in (4.35) completes the proof of Lemma 4.17

We are now ready to give the proof of Proposition 4.3.

Proof of Proposition 4.3. We recall that under the assumption of Proposition 4.3 there exists a unique solution $(v, d) \in X_{T_{*}}$ to the problem (2.13), see Theorem 3.7. We can assume that $T_{*}>0$ is the maximal time of existence of $(v, d)$. Let $r_{0}>0$ and $\varepsilon_{0}>0$ be as in Lemmas 4.1 and 4.9 , respectively. Let $R_{0} \in\left(0, r_{0}\right)$ be chosen such that

$$
\varepsilon_{1}^{2}=\mathcal{E}_{2 R_{0}}\left(v_{0}, d_{0}\right)=\frac{1}{2} \sup _{x \in \Omega} \int_{\Omega \cap B_{2 R_{0}}}\left(\left|v_{0}\right|^{2}+\left|\nabla d_{0}\right|^{2}+\phi\left(d_{0}\right)\right) \mathrm{d} x<\varepsilon_{0}^{2} .
$$

Let us observe that since $\mathcal{E}\left(v_{0}, d_{0}\right)<\infty$ and $\mu(A)=\int_{\Omega \cap A}\left[\left|v_{0}\right|^{2}+\left|\nabla d_{0}\right|^{2}+\phi\left(d_{0}\right)\right]$ $\mathrm{d} x$ is absolutely continuous, it is possible to choose such $R_{0}$. We also observe that $\varepsilon_{1}^{2}<E_{0}$. Now, let

$$
\theta_{0}\left(\varepsilon_{1}, E_{0}\right):=\min \left\{\frac{K_{4}^{-4} \varepsilon_{1}^{6}\left(1+r_{0}+\varepsilon_{1}\right)^{-4}}{\left[1+2 E_{0}+\Psi(T)+T\right]^{5}}, \frac{3}{4}\right\} .
$$

and $T_{0}=T\left(\varepsilon_{1}, R_{0}\right)$. By Corollary $4.14 T_{0}<T_{*}$. We will now distinguish two cases.

- If $T_{0}>R_{0}^{2}$, then because $\theta_{0}\left(\varepsilon_{1}, E_{0}\right) \in\left(0, \frac{3}{4}\right]$,

$$
T_{0} \geq \theta_{0}\left(\varepsilon_{1}, E_{0}\right) R_{0}^{2},
$$

- If $T_{0} \leq R_{0}^{2}$, then by Corollary 4.14 , the definition of $T_{0}$ and the continuity of $(v, d)$ at $t=T_{0}$ we infer that

$$
\mathcal{E}_{R_{0}}\left(v\left(T_{0}\right), d\left(T_{0}\right)\right)-\mathcal{E}_{2 R_{0}}\left(v_{0}, d_{0}\right)=2 \varepsilon_{1}^{2}-\varepsilon_{1}^{2}=\varepsilon_{1}^{2}
$$


Hence, by using the inequality (4.42) with $t=T_{0}$ and the fact $\varepsilon_{1}^{2}<E_{0}$, we infer the existence of a universal constant $K_{7}>0$ such that

$$
\begin{aligned}
\varepsilon_{1}^{2} & \leq K_{7} T_{0}^{\frac{1}{4}}\left(1+E_{0}+\Psi(T)+T+\frac{T_{0} E_{0}}{R_{0}^{2}}\right)^{\frac{5}{4}}\left(R_{0}^{-\frac{1}{2}}\left(\varepsilon_{1}^{\frac{3}{2}}+\varepsilon_{1}^{\frac{1}{2}}\right)+\varepsilon_{1}^{\frac{1}{2}}\right) \\
& \leq K_{7} \varepsilon_{1}^{\frac{1}{2}}\left(1+R_{0}^{-\frac{1}{2}}\left[1+\varepsilon_{1}\right]\right) T_{0}^{\frac{1}{4}}\left[1+2 E_{0}+\Psi(T)+T\right]^{\frac{5}{4}},
\end{aligned}
$$

where $\Psi$ is defined in (4.19). Since $R_{0} \leq r_{0}$, we have

$$
R_{0}^{\frac{1}{2}}+\varepsilon_{1}+1 \leq 1+r_{0}^{\frac{1}{2}}+\varepsilon_{1} .
$$

Hence,

$$
\varepsilon_{1}^{2} \leq K_{4} \varepsilon_{1}^{\frac{1}{2}} R_{0}^{-\frac{1}{2}}\left(1+r_{0}^{\frac{1}{2}}+\varepsilon_{1}\right) T_{0}^{\frac{1}{4}}\left[1+2 E_{0}+\Psi(T)+T\right]^{\frac{5}{4}},
$$

from which we deduce that

$$
T_{0} \geq \frac{K_{4}^{-4} \varepsilon_{1}^{6}\left(1+r_{0}^{\frac{1}{2}}+\varepsilon_{1}\right)^{-4}}{\left[1+2 E_{0}+\Psi(T)+T\right]^{5}} R_{0}^{2}=\theta_{0}\left(\varepsilon_{1}, E_{0}\right) R_{0}^{2} .
$$

By the definition of $T_{0}=T\left(\varepsilon_{1}, R_{0}\right)$, see (4.10), and the fact $T_{0}<T_{*}$ we automatically obtain (4.8). Thus, the proof of Proposition 4.3 is complete.

\section{The existence and the uniqueness of a global weak solution}

In this section we will prove global existence of a weak solutions to problem (2.13). Before we state and prove this result let us define the concept of a weak solution.

Definition 5.1. A global weak solution to (2.13) is a pair of functions $(v, d)$ : $[0, T) \rightarrow \mathrm{H} \times \mathrm{H}^{1}$ such that

(1) $(v, d) \in L^{\infty}\left(0, T ; \mathrm{H} \times \mathrm{H}^{1}\right)$

(2) for all $t \in[0, T)$ the following integral equations

$$
\begin{aligned}
v(t)=v_{0} & +\int_{0}^{t}[A v(s)-B(v(s))-\Pi(\operatorname{div}[\nabla d(s) \odot \nabla d(s)])] \mathrm{d} s+\int_{0}^{t} \Pi f(s) \mathrm{d} s, \\
d(t)=d_{0} & +\int_{0}^{t}\left[\Delta d(s)+|\nabla d(s)|^{2} d(s)-v(s) \cdot \nabla d(s)\right. \\
& \left.-\phi^{\prime}(d(s))+\left(\phi^{\prime}(d(s)) \cdot d(s)\right) d(s)\right] \mathrm{d} s \\
& +\int_{0}^{t}(d(s) \times g(s)) \mathrm{d} s
\end{aligned}
$$

hold in $D\left(A^{-\frac{3}{2}}\right)$ and $\mathrm{H}^{-2}$, respectively.

(3) For all $t \in[0, T) d(t) \in \mathcal{M}$, 
(4) and $\left(\partial_{t} v, \partial_{t} d\right) \in L^{2}\left(0, T ; D\left(A^{-\frac{3}{2}}\right) \times \mathrm{H}^{-2}\right)$.

We also introduce the notion of local strong solution which will be needed to prove the existence of a global weak solution to our problem.

Definition 5.2. Let $T_{0} \in(0, T]$. A function $(v, d):\left[0, T_{0}\right] \rightarrow H \times \mathrm{H}^{1}$ is a local strong solution to $(2.13)$ with initial data $(v(0), d(0))=\left(v_{0}, d_{0}\right)$ iff

(1) $(v, d) \in C\left(\left[0, T_{0}\right] ; \mathrm{H} \times \mathrm{H}^{1}\right) \cap L^{2}\left(0, T_{0} ; \mathrm{V} \times D(\hat{\mathrm{A}})\right)$,

(2) for all $t \in\left[0, T_{0}\right]$ the equations (3.4) and (3.5) hold in $\mathrm{V}^{*}$ and $L^{2}$, respectively.

(3) For all $t \in\left[0, T_{0}\right] d(t) \in \mathcal{M}$,

(4) and $\left(\partial_{t} v, \partial_{t} d\right) \in L^{2}\left(0, T_{0} ; \mathrm{V}^{*} \times L^{2}\right)$.

As usual we denote by $\left((v, d) ; T_{0}\right)$ a local strong solution defined on $\left[0, T_{0}\right]$.

Similarly to Definition 3.5, one we can also define the notion of a maximal local strong solution.

We state the following important remark.

Remark 5.3. From the definition it is clear that a maximal local solution $(v, d)$ defined on $\left[0, T_{0}\right)$ is a local solution on the open interval $\left[0, T_{0}\right)$.

In the definitions above, the condition $\left(\partial_{t} v, \partial_{t} d\right) \in L^{2}\left(0, T_{0} ; D\left(A^{-\frac{j+1}{2}}\right) \times \mathrm{H}^{j-2}\right)$, $j=0,2$, is equivalent to

$$
\begin{aligned}
& F(v, d)=-\Pi(v \cdot \nabla v)-\Pi(\operatorname{div}[\nabla d \odot \nabla d]) \in L^{2}\left(0, T_{0} ; D\left(A^{-\frac{j+1}{2}}\right)\right), j=0,2, \\
& G(v, d)=|\nabla d|^{2} d-v \cdot \nabla d-\phi^{\prime}(d)+\left(\phi^{\prime}(d) \cdot d\right) d \in L^{2}\left(0, T_{0} ; H^{j-2}\right), j=0,2 .
\end{aligned}
$$

Let us now state the standing assumptions of this section.

Assumption 5.4. Let $T>0$ and assume that $(f, g) \in L^{2}\left(0, T ; \mathrm{H}^{-1} \times L^{2}\right)$. We also assume that $\left(v_{0}, d_{0}\right) \in H \times \mathrm{H}^{1}$ satisfies

$$
\begin{aligned}
& E_{0}=\mathcal{E}\left(v_{0}, d_{0}\right)=\int_{\Omega}\left(\left|u_{0}\right|^{2}+\left|\nabla d_{0}\right|^{2}+\phi\left(d_{0}\right)\right) \mathrm{d} y<\infty, \\
& d_{0} \in \mathcal{M} .
\end{aligned}
$$

The main result of this section is the following uniqueness result.

Proposition 5.5. Let $\left(v_{i}, d_{i}\right) \in L^{\infty}\left(0, T ; \mathrm{H} \times \mathrm{H}^{1}\right) \cap L^{2}\left(0, T ; D\left(\mathrm{~A}^{\frac{1}{2}}\right) \times D\left(\hat{\mathrm{A}}^{\frac{3}{2}}\right)\right.$, $i=1,2$, be two weak solutions to (2.13) defined on $[0, T]$. Then,

$$
\left(v_{1}, d_{1}\right)=\left(v_{2}, d_{2}\right) .
$$

Proof. In order to prove this result we closely follow the approach of [24].

Let $\left(v_{i}, d_{i}\right)$ be a two strong solutions to (2.13), $v=v_{1}-v_{2}$ and $d=d_{1}-d_{2}$. Hence, $v$ satisfies the equation

$$
\frac{\mathrm{d} v}{\mathrm{~d} t}+\mathrm{A} v+B\left(v, v_{1}\right)+B\left(v_{2}, v\right)=-\Pi\left(\operatorname{div}\left[\nabla d \odot \nabla d_{1}+\nabla d_{2} \odot \nabla d\right]\right) .
$$


Let $w=\mathrm{A}^{-1} v$. It is not difficult to show that $w$ satisfies $\frac{\mathrm{d} w}{\mathrm{~d} t}+\mathrm{A} w+A^{-1}\left(B\left(v, v_{1}\right)+B\left(v_{2}, v\right)\right)=-\mathrm{A}^{-1} \Pi\left(\operatorname{div}\left[\nabla d \odot \nabla d_{1}+\nabla d_{2} \odot \nabla d\right]\right)$.

By parts (1) and (4) of Definition 5.2, we have $w \in L^{2}\left(0, T ; D\left(\mathrm{~A}^{\frac{3}{2}}\right)\right)$ and $\partial_{t} w=$ $A^{-1} \partial_{t} v \in L^{2}(0, T ; \mathrm{V}) \subset L^{2}\left(0, T ; D(\mathrm{~A})^{*}\right)$. Then, by applying the Lions-Magenes lemma ([37, Lemma III.1.2]) and using the facts that A is self-adjoint and $\operatorname{div} w=0$ we infer that

$$
\begin{aligned}
\frac{1}{2} \frac{\mathrm{d}}{\mathrm{d} t}\left|\mathrm{~A}^{\frac{1}{2}} w\right|_{L^{2}}^{2}+|\mathrm{A} w|_{L^{2}}^{2} & =-\left\langle B\left(v, v_{1}\right)+B\left(v_{1}, v\right), w\right\rangle-\left\langle\Pi\left(\operatorname{div}\left[\nabla d \odot \nabla d_{1}+\nabla d_{2} \odot \nabla d\right]\right), w\right\rangle \\
& =-\left\langle B\left(v, v_{1}\right)+B\left(v_{1}, v\right), w\right\rangle-\left\langle\nabla d \odot \nabla d_{1}+\nabla d_{2} \odot \nabla d, \nabla w\right\rangle .
\end{aligned}
$$

We also used the integration by parts to obtain the second line. Let us now estimate the terms on the right hand side of the last line of the chain of identities above.

Hereafter we fix $\varepsilon, \gamma>0$, the symbols $C_{\varepsilon}, C_{\varepsilon, \gamma}$ denote two positive constants depending only on $\varepsilon$ and $\gamma$.

Firstly, by using the Hölder, the Young inequalities and the Ladyzhenskaya inequality ([37, Lemma III.3.3]) we infer that

$$
\begin{aligned}
-\left\langle B\left(v, v_{1}\right), w\right\rangle & =\left\langle B(v, w), v_{1}\right\rangle \\
& \leq|v|_{L^{2}}|\nabla w|_{L^{4}}\left|v_{1}\right|_{L^{4}} \\
& \leq \varepsilon|v|_{L^{2}}^{2}+C_{\varepsilon}|\nabla w|_{L^{2}}\left|\nabla^{2} w\right|_{L^{2}}\left|v_{1}\right|_{L^{4}}^{2} \\
& \leq \varepsilon|v|_{L^{2}}^{2}+C_{\varepsilon}\left|\mathrm{A}^{\frac{1}{2}} w\right|_{L^{2}}|\mathrm{~A} w|_{L^{2}}\left|v_{1}\right|_{L^{4}}^{2} \\
& \leq \varepsilon|v|_{L^{2}}^{2}+\varepsilon|\mathrm{A} w|_{L^{2}}^{2}+C_{\varepsilon}\left|\mathrm{A}^{\frac{1}{2}} w\right|_{L^{2}}^{2}\left|v_{1}\right|_{L^{4}}^{2}
\end{aligned}
$$

Observe that $|v|_{L^{2}}^{2}=|\mathrm{A} w|_{L^{2}}^{2}$. Thus,

$$
-\left\langle B\left(v, v_{1}\right), w\right\rangle \leq 2 \varepsilon|\mathrm{A} w|_{L^{2}}^{2}+C_{\varepsilon}\left|\mathrm{A}^{\frac{1}{2}} w\right|_{L^{2}}^{2}\left|v_{1}\right|_{L^{4}}^{4}
$$

In a similar way, we can prove that

$$
-\left\langle B\left(v_{2}, v\right), w\right\rangle \leq 2 \varepsilon|\mathrm{A} w|_{L^{2}}^{2}+C_{\varepsilon}\left|\mathrm{A}^{\frac{1}{2}} w\right|_{L^{2}}^{2}\left|v_{2}\right|_{L^{4}}^{4}
$$

Secondly, making use of the Ladyzhenskaya inequality ([37, Lemma III.3.3]), the Hölder and the Young inequalities we obtain

$$
\begin{aligned}
-\left\langle\nabla d \odot \nabla d_{1}, \nabla w\right\rangle & \leq|\nabla d|_{L^{2}}\left|\nabla d_{1}\right|_{L^{4}}|\nabla w|_{L^{4}} \\
& \leq \gamma|\nabla d|_{L^{2}}^{2}+C_{\gamma}\left|\nabla d_{1}\right|_{L^{4}}^{2}|\nabla w|_{L^{2}}\left|\nabla^{2} w\right|_{L^{2}} \\
& \leq \gamma|\nabla d|_{L^{2}}^{2}+C_{\gamma}\left|\nabla d_{1}\right|_{L^{4}}^{2}\left|\mathrm{~A}^{\frac{1}{2}} w\right|_{L^{2}}|\mathrm{~A} w|_{L^{2}} \\
& \leq \gamma|\nabla d|_{L^{2}}^{2}+\varepsilon|\mathrm{A} w|_{L^{2}}^{2}+C_{\gamma, \varepsilon}\left|\mathrm{A}^{\frac{1}{2}} w\right|_{L^{2}}^{2}\left|\nabla d_{1}\right|_{L^{4}}^{4}
\end{aligned}
$$


Similarly,

$$
-\left\langle\nabla d_{2} \odot \nabla d, \nabla w\right\rangle \leq \gamma|\nabla d|_{L^{2}}^{2}+\varepsilon|\mathrm{A} w|_{L^{2}}^{2}+C_{\gamma, \varepsilon}\left|\mathrm{A}^{\frac{1}{2}} w\right|_{L^{2}}^{2}\left|\nabla d_{2}\right|_{L^{4}}^{4} \cdot
$$

Collecting all these inequalities we obtain

$$
\begin{aligned}
\frac{1}{2} \frac{\mathrm{d}}{\mathrm{d} t}\left|\mathrm{~A}^{\frac{1}{2}} w\right|_{L^{2}}^{2}+|\mathrm{A} w|_{L^{2}}^{2} \leq & \varepsilon|\mathrm{A} w|_{L^{2}}^{2}+\gamma|\nabla d|_{L^{2}}^{2} \\
& +C_{\varepsilon, \gamma}\left|\mathrm{A}^{\frac{1}{2}} w\right|_{L^{2}}^{2}\left(\left|v_{1}\right|_{L^{4}}^{4}+\left|v_{2}\right|_{L^{4}}^{4}+\left|\nabla d_{1}\right|_{L^{4}}^{4}+\left|\nabla d_{2}\right|_{L^{4}}^{4}\right) .
\end{aligned}
$$

Let us turn our attention to the function $d=d_{1}-d_{2}$. We notice that $d$ satisfies

$$
\begin{aligned}
\frac{\mathrm{d}}{\mathrm{d} t} d & +\hat{\mathrm{A}} d+v \cdot \nabla d_{1}+v_{2} \cdot \nabla d=\left(\left|\nabla d_{1}\right|^{2}-\left|\nabla d_{2}\right|^{2}\right) d_{1} \\
+ & \left|\nabla d_{2}\right|^{2} d-\left[\phi^{\prime}\left(d_{1}\right)-\phi^{\prime}\left(d_{2}\right)\right] \\
& +\left[\alpha\left(d_{1}\right)-\alpha\left(d_{2}\right)\right] d_{1}+\alpha\left(d_{2}\right) d+d \times g \\
= & \left(\nabla d_{1}-\nabla d_{2}: \nabla d_{1}+\nabla d_{2}\right) d_{1}+\left|\nabla d_{2}\right| d-\left[\phi^{\prime}\left(d_{1}\right)-\phi^{\prime}\left(d_{2}\right)\right] \\
& +\left[\alpha\left(d_{1}\right)-\alpha\left(d_{2}\right)\right] d_{1}+\alpha\left(d_{2}\right) d+d \times g \\
= & \left(\nabla d: \nabla d_{1}+\nabla d_{2}\right) d_{1}+\left|\nabla d_{2}\right| d-\left[\phi^{\prime}\left(d_{1}\right)-\phi^{\prime}\left(d_{2}\right)\right] \\
& +\left[\alpha\left(d_{1}\right)-\alpha\left(d_{2}\right)\right] d_{1}+\alpha\left(d_{2}\right) d+d \times g .
\end{aligned}
$$

Since $d_{1}, d_{2} \in L^{2}(0, T ; D(\hat{\mathrm{A}}))$, and $\partial_{t} d_{1}, \partial_{t} d_{2} \in L^{2}\left(0, T ; L^{2}\right)$, we infer by applying Lions-Magenes lemma ([37, Lemma III.1.2]), and the facts $\left\langle v_{2} \cdot \nabla d, d\right\rangle=0$ and $\langle d \times g, d\rangle=0$ (because $d \times g \perp_{\mathbb{R}^{3}} d$ ) that

$$
\begin{aligned}
\frac{1}{2} \frac{\mathrm{d}}{\mathrm{d} t}|d|_{L^{2}}^{2}+|\nabla d|_{L^{2}}^{2}= & -\left\langle v \cdot \nabla d_{1}+\left[\nabla d: \nabla\left(d_{1}+d_{2}\right)\right] d_{1}+\left|\nabla d_{2}\right|^{2} d\right. \\
& \left.-\left[\phi^{\prime}\left(d_{1}\right) \phi^{\prime}\left(d_{2}\right)\right]_{+} \alpha\left(d_{2}\right) d, d\right\rangle \\
& +\left\langle\left[\alpha\left(d_{1}\right)-\alpha\left(d_{2}\right)\right] d_{1}, d\right\rangle .
\end{aligned}
$$

Let us estimate the terms in the right hand side of (5.2). First, by using the Hölder, the Young inequalities and the Gagliardo-Nirenberg inequality ([1, Section 9.8, Example C.3]) we show that

$$
\begin{aligned}
-\left\langle v \cdot \nabla d_{1}, d\right\rangle & \leq|v|_{L^{2}}\left|\nabla d_{1}\right|_{L^{4}}|d|_{L^{4}} \\
& \leq \varepsilon|v|_{L^{2}}^{2}+C_{\varepsilon}\left|\nabla d_{1}\right|_{L^{4}}^{2}|d|_{L^{2}}\left(|d|_{L^{2}}+|\nabla d|_{L^{2}}\right) \\
& \leq \varepsilon|v|_{L^{2}}^{2}+\gamma|\nabla d|_{L^{2}}^{2}+C_{\varepsilon}\left|\nabla d_{1}\right|_{L^{4}}^{2}|d|^{2}+|d|_{L^{2}}^{2}\left|\nabla d_{1}\right|_{L^{4}}^{4} \\
& \leq \varepsilon|\mathrm{A} w|_{L^{2}}^{2}+\gamma|\nabla d|_{L^{2}}^{2}+C_{\varepsilon, \gamma}|d|_{L^{2}}^{2}\left(1+\left|\nabla d_{1}\right|_{L^{4}}^{4}\right)
\end{aligned}
$$

With the same idea, we prove that

$$
\left\langle\left[\nabla d: \nabla\left(d_{1}+d_{2}\right)\right] d_{1}, d\right\rangle \leq|\nabla d|_{L^{2}}|d|_{L^{4}}\left[\left|\nabla d_{1}\right|_{L^{4}}+\left|\nabla d_{2}\right|_{L^{4}}\right]\left|d_{1}\right|_{L^{\infty}}
$$




$$
\begin{aligned}
\leq & \gamma|\nabla d|_{L^{2}}^{2}+C_{\gamma}|d|_{L^{2}}\left[|d|_{L^{2}}\right. \\
& \left.+|\nabla d|_{L^{2}}\right]\left[\left|\nabla d_{1}\right|_{L^{4}}^{2}+\left|\nabla d_{2}\right|_{L^{4}}^{2}\right]\left|d_{1}\right|_{L^{\infty}}^{2} \\
\leq & 2 \gamma|\nabla d|_{L^{2}}^{2}+C_{\gamma}|d|_{L^{2}}^{2}\left[\left|\nabla d_{1}\right|_{L^{4}}^{2}\right. \\
& \left.+\left|\nabla d_{2}\right|_{L^{4}}^{2}+\left|\nabla d_{1}\right|_{L^{4}}^{4}+\left|\nabla d_{2}\right|_{L^{4}}^{4}\right] \\
\leq & 2 \gamma|\nabla d|_{L^{2}}^{2}+C_{\gamma}|d|_{L^{2}}^{2}\left[1+\left|\nabla d_{1}\right|_{L^{4}}^{4}+\left|\nabla d_{2}\right|_{L^{4}}^{4}\right]
\end{aligned}
$$

In the last line we used the fact that $\left|d_{1}\right|_{L^{\infty}} \leq 1$.

Utilizing the Hölder, the Young inequalities and the Gagliardo-Nirenberg inequality ([1, Section 9.8, Example C.3]) we obtain

$$
\begin{aligned}
\left\langle\left|\nabla d_{2}\right|^{2} d, d\right\rangle & \leq\left|\nabla d_{2}\right|_{L^{4}}^{2}|d|_{L^{4}}^{2} \\
& \leq\left|\nabla d_{2}\right|_{L^{4}}^{2}|d|_{L^{2}}\left(|d|_{L^{2}}+|\nabla d|_{L^{2}}\right) \\
& \leq \gamma|\nabla d|_{L^{2}}^{2}+C_{\gamma}|d|_{L^{2}}^{2}\left(1+\left|\nabla d_{2}\right|_{L^{4}}^{4}\right) .
\end{aligned}
$$

Since $\left|\phi^{\prime \prime}\right| \leq M$, the map $\phi^{\prime}: \mathbb{R}^{3} \rightarrow \mathbb{R}^{3}$ is Lipschitz and

$$
-\left\langle\phi^{\prime}\left(d_{1}\right)-\phi^{\prime}\left(d_{2}\right), d\right\rangle \leq M|d|_{L^{2}}^{2} .
$$

Using the definition of $\alpha\left(d_{2}\right)=\left(\phi^{\prime}\left(d_{2}\right) \cdot d_{2}\right)$, the fact $\left|d_{2}\right|=1$ and (3.1) we have

$$
\left\langle\alpha\left(d_{2}\right) d, d\right\rangle=\left\langle\left(\phi^{\prime}\left(d_{2}\right) \cdot d_{2}\right) d, d\right\rangle \leq 2 M|d|_{L^{2}}^{2} .
$$

Using again the definition of $\alpha\left(d_{1}\right)$ and $\alpha\left(d_{2}\right)$ we obtain

$$
\begin{aligned}
\left\langle\left[\alpha\left(d_{1}\right)-\alpha\left(d_{2}\right)\right] d_{1}, d\right\rangle & =\left\langle\left[\phi^{\prime}\left(d_{1}\right) \cdot d_{1}-\phi^{\prime}\left(d_{2}\right) \cdot d_{2}\right] d_{1}, d\right\rangle \\
& =\left\langle\left(\left[\phi^{\prime}\left(d_{1}\right)-\phi^{\prime}\left(d_{2}\right)\right] \cdot d_{1}+\phi^{\prime}\left(d_{2}\right) \cdot d\right) d_{1}, d\right\rangle .
\end{aligned}
$$

Since $\phi^{\prime}$ is Lipschitz, $d_{i}(t) \in \mathcal{M}$ for all $t \in[0, T]$, we show with the same ideas as used in (5.3) and (5.4) that

$$
\left\langle\left[\alpha\left(d_{1}\right)-\alpha\left(d_{2}\right)\right] d_{1}, d\right\rangle \leq 3 M|d|_{L^{2}}^{2} .
$$

Hence, collecting all these inequalities related to the terms in the right hand side of the equation (5.2) we obtain

$$
\frac{1}{2} \frac{\mathrm{d}}{\mathrm{d} t}|d|_{L^{2}}^{2}+|\nabla d|_{L^{2}}^{2} \leq \varepsilon|\mathrm{A} w|_{L^{2}}^{2}+\gamma|\nabla d|_{L^{2}}^{2}+C_{\gamma}|d|_{L^{2}}^{2}\left(1+\left|\nabla d_{1}\right|_{L^{4}}^{4}+\left|\nabla d_{2}\right|_{L^{4}}^{4}\right) .
$$

Thus, summing (5.1) and (5.5) up, we have

$$
\begin{aligned}
\frac{1}{2} \frac{\mathrm{d}}{\mathrm{d} t}\left(|d d|_{L^{2}}^{2}+\left|\mathrm{A}^{\frac{1}{2}} w\right|_{L^{2}}^{2}\right)+|\mathrm{A} w|_{L^{2}}^{2}+|\nabla d|_{L^{2}}^{2} \leq & \varepsilon|\mathrm{A} w|_{L^{2}}^{2}+\gamma|\nabla d|_{L^{2}}^{2} \\
& +C_{\gamma, \varepsilon}|d|_{L^{2}}^{2}\left(1+\left|\nabla d_{1}\right|_{L^{4}}^{4}+\left|\nabla d_{2}\right|_{L^{4}}^{4}\right) \\
& +C_{\varepsilon, \gamma}\left|\mathrm{A}^{\frac{1}{2}} w\right|_{L^{2}}^{2}\left(\left|\nabla d_{1}\right|_{L^{4}}^{4}+\left|\nabla d_{2}\right|_{L^{4}}^{4}\right. \\
& \left.+\left|v_{1}\right|_{L^{4}}^{4}+\left|v_{2}\right|_{L^{4}}^{4}\right) .
\end{aligned}
$$


Let choose $\varepsilon=\gamma=\frac{1}{2}$ and put

$$
\begin{aligned}
& y(t)=|d(t)|_{L^{2}}^{2}+\left|\mathrm{A}^{\frac{1}{2}} w(t)\right|_{L^{2}}^{2}, t \in[0, T], \\
& \Phi(t)=2 C_{\frac{1}{2}, \frac{1}{2}}\left(1+\left|\nabla d_{1}(t)\right|_{L^{4}}^{4}+\left|\nabla d_{2}(t)\right|_{L^{4}}^{4}+\left|v_{1}(t)\right|_{L^{4}}^{4}+\left|v_{2}(t)\right|_{L^{4}}^{4}\right), t \in[0, T] .
\end{aligned}
$$

Then, we see from (5.6) that $y$ satisfies

$$
\dot{y}(t) \leq \Phi(t) y(t), t \in[0, T] .
$$

Observe that since $\left(v_{i}, d_{i}\right) \in C([0, T], \mathrm{H} \times \mathrm{H}) \cap L^{2}\left(0, T ; D\left(\mathrm{~A}^{\frac{1}{2}}\right) \times D(\hat{\mathrm{A}})\right)$ we infer that

$$
\int_{0}^{T} \Phi(s) \mathrm{d} s<\infty .
$$

Thus, one can apply the Gronwall inequality to (5.7) and deduce that

$$
y(t) \leq y(0) e^{\int_{0}^{t} \Phi(s) \mathrm{d} s} \leq y(0) e^{\int_{0}^{T} \Phi(s) \mathrm{d} s}, t \in[0, T] .
$$

Since

$$
y(0)=\left|d_{1}(0)-d_{2}(0)\right|_{L^{2}}^{2}+\left|\mathrm{A}^{-1 \frac{1}{2}} v_{1}(0)-\mathrm{A}^{-\frac{1}{2}} v_{2}(0)\right|_{L^{2}}^{2}=0,
$$

we have

$$
y(t)=\left|d_{1}(t)-d_{2}(t)\right|_{L^{2}}^{2}+\left|v_{1}(t)-v_{2}(t)\right|_{D\left(\mathrm{~A}^{-\frac{1}{2}}\right)}^{2}=0, t \in[0, T] .
$$

This completes the proof of Proposition 5.5.

The main result of this paper is the following theorem.

Theorem 5.6. Let $\left(v_{0}, d_{0}\right) \in \mathrm{H} \times \mathrm{H}^{1}, r_{0}>0$ and $\varepsilon_{0}>0$ be the constants from Lemmas 4.1 and 4.9 , respectively. Then, there exist constants $\varrho_{0} \in\left(0, r_{0}\right]$ and $\varepsilon_{1} \in$ $\left(0, \varepsilon_{0}\right)$ such that the following hold.

If Assumption 5.4 holds, then there are a number $L \in \mathbb{N}$, depending only on the norms of $\left(v_{0}, d_{0}\right) \in H \times \mathrm{H}^{1}$ and $(f, g) \in L^{2}\left(0, T ; \mathrm{H}^{-1} \times L^{2}\right)$, a collection of times $0<T_{1}<\cdots<T_{L} \leq T$ and a unique global weak solution $(\mathbf{v}, \mathbf{d}) \in$ $C_{w}\left([0, T] ; \mathrm{H} \times \mathrm{H}^{1}\right) \cap L^{2}(0, T ; \mathrm{V} \times D(\hat{\mathrm{A}}))$ to $(1.2)$ such that

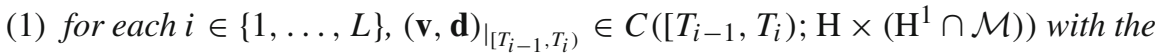
left-limit at $T_{i}$, is a maximal local regular solution to (2.13) with initial data $\left(v\left(T_{i-1}\right), d\left(T_{i-1}\right)\right)$. Here we understand that $T_{0}=0$.

(2) If $T_{L}<T$, then $(\mathbf{v}, \mathbf{d})_{\left.\right|_{\left[T_{L}, T\right]}}$ belongs to $C\left(\left[T_{L}, T\right] ; \mathrm{H} \times \mathrm{H}^{1}\right)$ and satisfies the variational form problem (1.2) on the interval $\left[T_{L}, T\right]$ with initial data $\left(v\left(T_{L}\right), d\left(T_{L}\right)\right)$.

(3) For each $i \in\{1, \ldots, L\}$

$$
\lim _{t \nearrow T_{i}} \mathcal{E}_{R}(\mathbf{v}(t), \mathbf{d}(t)) \geq \varepsilon_{1}^{2}
$$

for all $R \in\left(0, \varrho_{0}\right]$. 
(4) At each $T_{i}$ there is a loss of energy at least $\varepsilon_{1}^{2} \in\left(0, \varepsilon_{0}^{2}\right)$, i.e., $\mathcal{E}\left(\mathbf{v}\left(T_{i}\right), \mathbf{d}\left(T_{i}\right)\right) \leq \mathcal{E}\left(\mathbf{v}\left(T_{i-1}\right), \mathbf{d}\left(T_{i-1}\right)\right)+\frac{1}{2} \int_{T_{i-1}}^{T_{1}}\left[|f(t)|_{\mathrm{H}^{-1}}^{2}+|g(t)|_{L^{2}}^{2}\right] \mathrm{d} t-\varepsilon_{1}^{2}$.

Remark 5.7. Note that since $(\mathbf{v}, \mathbf{d}) \in C_{w}\left([0, T] ; \mathrm{H} \times \mathrm{H}^{1}\right)$, it holds that $(\mathbf{v}, \mathbf{d}) \in$ $L^{\infty}\left(0, T ; \mathrm{H} \times \mathrm{H}^{1}\right)$. Hence, the uniqueness of the weak solutions follows from Proposition 5.5.

The proof of existence this theorem is established in several steps. The first of such steps is the proof of the existence of a maximal local strong solution for the Ericksen-Leslie system (2.13) with data satisfying Assumption 5.4.

Theorem 5.8. Let $\left(v_{0}, d_{0}\right) \in \mathrm{H} \times\left(\mathrm{H}^{1} \cap \mathcal{M}\right), r_{0}>0$ and $\varepsilon_{0}>0$ be the constants from Lemmas 4.1 and 4.9 , respectively. Then, there exist $\varrho_{0} \in\left(0, r_{0}\right]$ such that

$$
\varepsilon_{1}^{2}=\mathcal{E}_{2 \varrho_{0}}\left(v_{0}, d_{0}\right)<\varepsilon_{0}^{2},,
$$

and a maximal local strong solution $\left((v, d) ; T_{*}\right)$ satisfying

$$
\limsup _{t \nearrow T_{*}} \mathcal{E}_{R}(v(t), d(t)) \geq \varepsilon_{1}^{2},
$$

for all $R \in\left(0, \varrho_{0}\right]$.

For the proof of this theorem, we first prove the existence of a local strong solution which is given by the following proposition.

Proposition 5.9. There exist $\varepsilon_{0}>0$ and a function

$$
\theta_{0}:\left(0, \varepsilon_{0}\right) \times(0, \infty) \rightarrow\left(0, \frac{3}{4}\right]
$$

which is non-increasing w.r.t. the second variable and non-decreasing w.r.t. the first one such that the following holds.

Let $r_{0}>0$ be the constant from Lemma 4.1 and assume that the initial data $\left(v_{0}, d_{0}\right)$ and the forcing $(f, g)$ satisfies Assumption 5.4.

Then, there exists $\varrho_{0} \in\left(0, r_{0}\right]$ such that

$$
\varepsilon_{1}^{2}=2 \mathcal{E}_{2 \varrho_{0}}\left(v_{0}, d_{0}\right)<\varepsilon_{0}^{2} .
$$

Moreover, there exists a local strong solution $\left((v, d) ; T_{0}\right)$ satisfying

$$
\begin{gathered}
T_{0} \geq \frac{\varrho_{0}^{2}}{\left(\varrho_{0}^{\frac{1}{2}}+1\right)^{4}} \theta_{0}\left(\varepsilon_{1}, E_{0}\right) \\
\sup _{t \in\left[0, T_{0}\right]} \mathcal{E}_{\varrho_{0}}(v(t), d(t)) \leq 2 \varepsilon_{1}^{2} .
\end{gathered}
$$

Furthermore, there exists a constant $K_{5}>0$ 


$$
\begin{aligned}
& \sup _{t \in\left[0, T_{0}\right]} \mathcal{E}(v(t), d(t))+\int_{0}^{T_{0}}|\nabla v(r)|_{L^{2}}^{2} d r \leq E_{0}+\frac{1}{2} \int_{0}^{T}\left(|f(s)|_{\mathrm{H}^{-1}}^{2}+\mid g\left(\left.s\right|_{L^{2}} ^{2}\right) \mathrm{d} s\right. \\
& \int_{0}^{T_{0}}|\Delta d(t)|_{L^{2}}^{2} \mathrm{~d} t \leq K_{5}\left[E_{0}+\frac{1}{2} \int_{0}^{T}\left(|f(s)|_{\mathrm{H}^{-1}}^{2}+|g(s)|_{L^{2}}^{2}\right) \mathrm{d} s+\left(1+\frac{E_{0}}{R^{2}}\right) T\right], \quad R \in\left(0, \varrho_{0}\right]
\end{aligned}
$$

where $E_{0}:=\mathcal{E}\left(u_{0}, d_{0}\right)$.

Proof of Proposition 5.9. There exists a sequence $\left\{d_{0}^{k}\right\}_{k=1}^{\infty} \subset C^{\infty}\left(\Omega ; \mathbb{S}^{2}\right)$ such that

$$
\lim _{k \rightarrow \infty}\left\|d_{0}^{k}-d_{0}\right\|_{H^{1}}=0
$$

see [32, Section 4]. By definition, $v_{0} \in \mathrm{H}$ can be approximated by a sequence $\left\{v_{0}^{k}\right\}_{k=1}^{\infty} \subset \mathcal{V}$ such that

$$
\lim _{k \rightarrow \infty}\left|v_{0}^{k}-v_{0}\right|_{L^{2}}=0
$$

Since embedding $L^{2} \hookrightarrow \mathrm{H}^{-1}$ and $\mathrm{H}^{1} \subset L^{2}$ are dense, then one can also approximate $(f, g) \in L^{2}\left(0, T ; \mathrm{H}^{-1} \times L^{2}\right)$ by a sequence $\left(\left(f_{k}, g_{k}\right)\right)_{k \in \mathbb{N}} \subset L^{2}\left(0, T ; L^{2} \times \mathrm{H}^{1}\right)$ in the following sense

$$
\left(f_{k}, g_{k}\right) \rightarrow(f, g) \text { strong in } L^{2}\left(0, T ; \mathrm{H}^{-1} \times L^{2}\right) .
$$

Let $\varepsilon_{0}>0$ and $R_{0} \in\left(0, r_{0}\right]$ be the constants from Proposition 4.3. Since $\mathcal{E}\left(v_{0}, d_{0}\right)<\infty$ and $\mu(A)=\int_{\Omega \cap A}\left[\left|v_{0}\right|^{2}+\left|\nabla d_{0}\right|^{2}+\phi\left(d_{0}\right)\right] \mathrm{d} x$ is absolutely continuous, there exists $\tilde{R}_{0}>0$ such that

$$
\varepsilon_{1}^{2}:=\sup _{x \in \Omega} \int_{\Omega \cap B_{2 \tilde{R_{0}}}}\left(\left|v_{0}\right|^{2}+\left|\nabla d_{0}\right|^{2}+\phi\left(d_{0}\right)\right) \mathrm{d} x<\varepsilon_{0}^{2} .
$$

Choosing $\varrho_{0}=\tilde{R}_{0} \wedge R_{0}$ yields (5.9). Let $\varepsilon>0$ be an arbitrary real number. Since $\left(v_{0}^{k}, d_{0}^{k}\right)$ strongly converges to $\left(v_{0}, d_{0}\right)$ in $L^{2} \times \mathrm{H}^{1}$, there exists a number $k_{0} \in \mathbb{N}$ such that for all $k \geq k_{0}$

$$
\begin{aligned}
\frac{1}{2} \int_{\Omega \cap B_{2 \varrho_{0}}(x)}\left|v_{0}^{k}\right|^{2} \mathrm{~d} x & =\frac{1}{2} \int_{\Omega \cap B_{2 \varrho_{0}}(x)}\left|v_{0}^{k}-v_{0}+v_{0}\right|^{2} \\
& \leq \int_{\Omega \cap B_{2 \varrho_{0}(x)}}\left|v_{0}^{k}-v_{0}\right|^{2} \mathrm{~d} x+\int_{\Omega \cap B_{2 \varrho_{0}(x)}}\left|v_{0}\right|^{2} \mathrm{~d} x \\
& \leq \frac{\varepsilon}{3}+\int_{\Omega \cap B_{2 \varrho_{0}(x)}}\left|v_{0}\right|^{2} \mathrm{~d} x
\end{aligned}
$$

In a similar way one can prove that,

$$
\int_{\Omega \cap B_{2 \varrho_{0}}(x)}\left|\nabla d_{0}^{k}\right|^{2} \mathrm{~d} x \leq \frac{\varepsilon}{3}+\int_{\Omega \cap B_{2 \varrho_{0}(x)}}\left|\nabla d_{0}\right|^{2} \mathrm{~d} x .
$$


Observe that

$$
\begin{aligned}
\int_{\Omega \cap B_{2 \varrho_{0}}(x)} \phi\left(d_{0}^{k}\right) \mathrm{d} x & =\int_{\Omega \cap B_{2 \varrho_{0}}(x)}\left(\phi\left(d_{0}^{k}\right)-\phi\left(d_{0}\right)+\phi\left(d_{0}\right)\right) \mathrm{d} x \\
& \leq \int_{\Omega \cap B_{2 \varrho_{0}}(x)}\left|\phi\left(d_{0}^{k}\right)-\phi\left(d_{0}\right)\right| \mathrm{d} x+\int_{\Omega \cap B_{2 \varrho_{0}}(x)} \phi\left(d_{0}\right) \mathrm{d} x \\
& \leq \int_{\Omega \cap B_{2 \varrho_{0}}(x)}\left|\phi\left(d_{0}^{k}\right)-\phi\left(d_{0}\right)\right| \mathrm{d} x+\int_{\Omega \cap B_{2 \varrho_{0}}(x)} \phi\left(d_{0}\right) \mathrm{d} x \\
& \leq \int_{\Omega \cap B_{2 \varrho_{0}}(x)}\left|\phi\left(d_{0}^{k}\right)-\phi\left(d_{0}\right)\right| \mathrm{d} x+\int_{\Omega \cap B_{2 \varrho_{0}}(x)} \phi\left(d_{0}\right) \mathrm{d} x
\end{aligned}
$$

Since $\left|d_{0}^{k}\right|=\left|d_{0}\right|=1$,

$$
\sup _{n \in \mathbb{S}^{2}}\left|\phi^{\prime}(n)\right| \leq M
$$

and $d_{0}^{k} \rightarrow d_{0}$ in $\mathrm{H}^{1}$ we deduce that there exists $k_{0} \in \mathbb{N}$ such that for all $k \geq k_{0}$

$$
\begin{aligned}
\int_{\Omega \cap B_{2 \varrho_{0}}(x)} \phi\left(d_{0}^{k}\right) \mathrm{d} x & \leq M \int_{\Omega}\left|d_{0}^{k}-d_{0}\right| \mathrm{d} x+\int_{\Omega \cap B_{2 \varrho_{0}}(x)} \phi\left(d_{0}\right) \mathrm{d} x \\
& \leq \frac{\varepsilon}{3}+\int_{\Omega \cap B_{2 \varrho_{0}}(x)} \phi\left(d_{0}\right) \mathrm{d} x
\end{aligned}
$$

Hence, there exists a constant $k_{0} \in \mathbb{N}$ such that for all $k \geq k_{0}$

$$
\mathcal{E}_{2 \varrho_{0}}\left(v_{0}^{k}, d_{0}^{k}\right) \leq \varepsilon+2 \mathcal{E}_{2 \varrho_{0}}\left(v_{0}, d_{0}\right)
$$

Since $\varepsilon$ is arbitrary, we infer that

$$
\mathcal{E}_{2 \varrho_{0}}\left(v_{0}^{k}, d_{0}^{k}\right) \leq \varepsilon_{1}^{2}<\varepsilon_{0}^{2} .
$$

Without loss of generality, we will assume that for all $k \geq 1$

$$
\mathcal{E}_{2 \varrho_{0}}\left(v_{0}^{k}, d_{0}^{k}\right) \leq \varepsilon_{1}^{2} .
$$

By Proposition 4.3 there exist a function $\theta_{0}:\left(0, \varepsilon_{0}\right) \times[0, \infty) \rightarrow\left(0, \frac{3}{4}\right]$ satisfying the properties stated in Proposition 5.9, a sequence of time $T_{0}^{k}$ satisfying (5.10) and a sequence of regular solutions $\left(v^{k}, d^{k}\right):\left[0, T_{0}^{k}\right] \rightarrow \mathrm{V} \times \mathrm{H}^{2}$, with initial condition

$$
\left(v_{0}^{k}(0), d_{0}^{k}(0)\right)=\left(v_{0}^{k}, d_{0}^{k}\right) .
$$

Moreover,

$$
\sup _{t \in\left[0, T_{0}^{k}\right]} \mathcal{E}_{\varrho_{0}}\left(v^{k}(t), d^{k}(t)\right) \leq 2 \varepsilon_{1}^{2} .
$$


We recall that for all $k \geq 1$ and $t \in\left[0, T_{0}^{k}\right)$, we have

$$
\begin{array}{rl}
\sup _{t \leq T_{0}^{k}} & \mathcal{E}\left(v^{k}(t), d^{k}(t)\right)+\int_{0}^{T_{0}^{k}}\left[\left|\nabla v^{k}(t)\right|_{L^{2}}^{2}+\mid \Delta d^{k}(t)-\phi^{\prime}\left(d^{k}(t)\right)+\alpha\left(d^{k}(t)\right) d^{k}(t)\right. \\
& \left.+\left.\left|\nabla d^{k}(t)\right|^{2} d^{k}(t)\right|_{L^{2}} ^{2}\right] \mathrm{d} t \\
& \leq \mathcal{E}\left(v_{0}^{k}, d_{0}^{k}\right)+\frac{1}{2} \int_{0}^{T_{0}^{k}}\left(\left|f_{k}(t)\right|_{\mathrm{H}^{-1}}^{2}+\left|g_{k}(t)\right|_{L^{2}}^{2}\right) \mathrm{d} t \leq E_{0}+\frac{1}{2} \int_{0}^{T_{0}^{k}}\left(|f(t)|_{\mathrm{H}^{-1}}^{2}\right. \\
& \left.+|g(t)|_{L^{2}}^{2}\right) \mathrm{d} t .
\end{array}
$$

Hereafter, we put

$$
\Xi_{0}=E_{0}+\frac{1}{2} \int_{0}^{T_{0}^{k}}\left(|f(t)|_{\mathrm{H}^{-1}}^{2}+|g(t)|_{L^{2}}^{2}\right) \mathrm{d} t .
$$

We also recall that there exists $C>0$ such that for all $k \in \mathbb{N}$ and $R \in\left(0, \varrho_{0}\right]$

$$
\begin{aligned}
& \int_{0}^{T_{0}^{k}}\left|\Delta d^{k}(t)\right|_{L^{2}}^{2} \mathrm{~d} t \leq C\left[E_{0}+\frac{1}{2} \int_{0}^{T}\left(|f(s)|_{\mathrm{H}^{-1}}^{2}+|g(s)|_{L^{2}}^{2}\right) \mathrm{d} s+\left(1+\frac{E_{0}}{R^{2}}\right) T\right] \\
& \int_{0}^{T_{0}^{k}}\left(\left|v^{k}(t)\right|_{L^{4}}^{4}+\left|\nabla d^{k}(t)\right|_{L^{4}}^{4}\right) \mathrm{d} t \leq C \varepsilon_{1}^{2}\left[E_{0}+\frac{1}{2} \int_{0}^{T}\left(|f(s)|_{\mathrm{H}^{-1}}^{2}+|g(s)|_{L^{2}}^{2}\right) \mathrm{d} s\right. \\
& \left.+\left(1+\frac{E_{0}}{R^{2}}\right) T\right], \\
& \int_{0}^{T_{0}^{k}}\left|\nabla v^{k}(t)\right|_{L^{2}}^{2} \mathrm{~d} t \leq E_{0}+\frac{1}{2} \int_{0}^{T}\left(|f(s)|_{\mathrm{H}^{-1}}^{2}+|g(s)|_{L^{2}}^{2}\right) \mathrm{d} s,
\end{aligned}
$$

and

$$
\sup _{t \leq T_{0}^{k}}\left[\left|v^{k}(t)\right|_{L^{2}}^{2}+\left|\nabla d^{k}(t)\right|_{L^{2}}^{2}+\int_{\Omega} \phi\left(d^{k}(t)\right) \mathrm{d} x\right] \leq E_{0}+\frac{1}{2} \int_{0}^{T}\left(|f(s)|_{\mathrm{H}^{-1}}^{2}+|g(s)|_{L^{2}}^{2}\right) \mathrm{d} s .
$$

We now estimate the time derivatives. Let us put

$$
\mathcal{E}(d(s))=\frac{1}{2}|\nabla d(s)|_{L^{2}}^{2}+\int_{\Omega} \phi(d(s, x)) \mathrm{d} x, s \in\left[0, T_{0}^{k}\right] .
$$

Then, we deduce from Claim 4.5 that

$$
\begin{aligned}
\int_{0}^{T_{0}^{k}}\left|\partial_{s} d^{k}(s)\right|_{L^{2}}^{2} \mathrm{~d} s & =\int_{0}^{T_{0}^{k}}\left\langle R(d(s)), \partial_{s} d(s)\right\rangle \mathrm{d} s-\int_{0}^{T_{0}^{k}}\left\langle v^{k}(s) \cdot \nabla d^{k}(s), \partial_{s} d^{k}(s)\right\rangle \mathrm{d} s \\
& =\int_{0}^{T_{0}^{k}} \frac{\mathrm{d}}{\mathrm{d} s} \mathcal{E}\left(d^{k}(s)\right) \mathrm{d} s-\int_{0}^{T_{0}^{k}}\left(v^{k}(s) \cdot \nabla d^{k}(s), \partial_{s} d^{k}(s)\right) \mathrm{d} s .
\end{aligned}
$$




\section{Hence}

$$
\begin{aligned}
\int_{0}^{T_{0}^{k}}\left|\partial_{s} d^{k}(s)\right|_{L^{2}}^{2} \mathrm{~d} s & \leq \sup _{t \leq T_{0}^{k}} \mathcal{E}\left(d^{k}(t)\right)-\mathcal{E}\left(d^{k}(0)\right)+\int_{0}^{T_{0}^{k}}\left(v^{k}(s) \cdot \nabla d^{k}(s), \partial_{s} d^{k}(s)\right) \mathrm{d} s \\
& \leq \mathcal{E}\left(v_{0}^{k}, d_{0}^{k}\right)-\mathcal{E}\left(d^{k}(0)\right)+\int_{0}^{T_{0}^{k}}\left(v^{k}(s) \cdot \nabla d^{k}(s), \partial_{s} d^{k}(s)\right) \mathrm{d} s \\
& \leq \frac{1}{2}\left|v_{0}^{k}\right|_{L^{2}}^{2}+\int_{0}^{T_{0}^{k}}\left(v^{k}(s) \cdot \nabla d^{k}(s), \partial_{s} d^{k}(s)\right) \mathrm{d} s \\
& \leq E_{0}+\int_{0}^{T_{0}^{k}}\left(v^{k}(s) \cdot \nabla d^{k}(s), \partial_{s} d^{k}(s)\right) \mathrm{d} s .
\end{aligned}
$$

Now we estimate the integral on the right hand side of the last line as follows:

$$
\begin{aligned}
\int_{0}^{T_{0}^{k}}\left(v^{k}(s) \cdot \nabla d^{k}(s), \partial_{s} d^{k}(s)\right) \mathrm{d} s & \leq \int_{0}^{T_{0}^{k}}\left|v^{k}(s) \cdot \nabla d^{k}(s)\right|_{L^{2}}\left|\partial_{s} d^{k}(s)\right|_{L^{2}} \mathrm{~d} s \\
& \leq \frac{1}{2} \int_{0}^{T_{0}^{k}}\left|\partial_{s} d^{k}(s)\right|_{L^{2}}^{2} \mathrm{~d} s+\frac{1}{2} \int_{0}^{T_{0}^{k}}\left|v^{k}(s) \cdot \nabla d^{k}(s)\right|_{L^{2}}^{2} \mathrm{~d} s \\
& \leq \frac{1}{2}\left(\int_{0}^{T_{0}^{k}}\left|v^{k}(s)\right|_{L^{4}}^{4} \mathrm{~d} s\right)^{\frac{1}{2}}\left(\int_{0}^{T_{0}^{k}}\left|\nabla d^{k}(s)\right|_{L^{4}}^{4} \mathrm{~d} s\right)^{\frac{1}{2}} \\
& +\frac{1}{2} \int_{0}^{T_{0}^{k}}\left|\partial_{s} d^{k}(s)\right|_{L^{2}}^{2} \mathrm{~d} s .
\end{aligned}
$$

By employing (5.16) in the last line we get

$$
\int_{0}^{T_{0}^{k}}\left(v^{k}(t) \cdot \nabla d^{k}(t), \partial_{t} d^{k}(t)\right) \mathrm{d} t \leq \frac{1}{2} \int_{0}^{T_{0}^{k}}\left|\partial_{t} d^{k}(t)\right|_{L^{2}}^{2}+\frac{C}{2} \varepsilon_{1}^{2} \Xi_{0} .
$$

Summing up these discussion, we get

$$
\sup _{k \in \mathbb{N}} \int_{0}^{T_{0}^{k}}\left|\partial_{t} d^{k}(t)\right|_{L^{2}}^{2} \mathrm{~d} t \leq E_{0}+\frac{1}{2} \sup _{k \in \mathbb{N}} \int_{0}^{T_{0}^{k}}\left|\partial_{t} d^{k}\right|_{L^{2}}^{2} \mathrm{~d} t+C \varepsilon_{1}^{2} \Xi_{0} .
$$

Thus, we obtain the following uniform estimate of $\partial_{t} d$

$$
\sup _{k \in \mathbb{N}} \int_{0}^{T_{0}^{k}}\left|\partial_{t} d^{k}(t)\right|_{L^{2}}^{2} \mathrm{~d} t \leq 2 E_{0}+2 C \varepsilon_{1}^{2} \Xi_{0} .
$$

We now estimate the time derivative $\partial_{t} v^{k}$. Let $\varphi \in V$. We have

$$
\left(\partial_{t} v^{k}, \varphi\right)=-\left(\nabla v^{k}, \nabla \varphi\right)-\int_{\Omega} v^{k} \cdot \nabla v^{k} \varphi \mathrm{d} x-\int_{\Omega} \operatorname{Div}\left(\nabla d^{k} \odot \nabla d^{k}\right) \varphi \mathrm{d} x+\int_{\Omega} f \mathrm{~d} x .
$$

Hence

$$
\left|\left(\partial_{t} v^{k}, \varphi\right)\right| \leq\left|\nabla v^{k}\right|_{L^{2}}|\nabla \varphi|_{L^{2}}+\int_{\Omega}\left|v^{k}\right|^{2}|\nabla \varphi| \mathrm{d} x
$$




$$
\begin{aligned}
& +\int_{\Omega}\left|\nabla d^{k}\right|^{2}|\nabla \varphi| \mathrm{d} x \\
\leq & |\nabla \varphi|_{L^{2}}\left[\left|\nabla v^{k}\right|_{L^{2}}+\left|v^{k}\right|_{L^{4}}^{2}+\left|\nabla d^{k}\right|_{L^{4}}^{2}+|f|_{L^{2}}\right]
\end{aligned}
$$

This altogether with (5.16) and (5.17) imply that there exists $C>0$ such that for all $k \in \mathbb{N}$

$$
\begin{aligned}
\sup _{k \in \mathbb{N}} \int_{0}^{T_{0}^{k}}\left|\partial_{t} v^{k}(s)\right|_{\mathrm{V}^{*}}^{2} \mathrm{~d} s \leq & 4\left[\int_{0}^{T_{0}^{k}}\left|\nabla v^{k}(s)\right|_{L^{2}}^{2} \mathrm{~d} s+\int_{0}^{T_{0}^{k}}\left|v^{k}(s)\right|_{L^{4}}^{4} \mathrm{~d} s+\int_{0}^{T_{0}^{k}}\left|\nabla d^{k}(s)\right|_{L^{4}}^{4} \mathrm{~d} s\right. \\
& \left.+\int_{0}^{T_{0}^{k}}|f(s)|_{L^{2}}^{2} \mathrm{~d} s\right] \leq C .
\end{aligned}
$$

Now, let us set

$$
T_{0}=\inf _{k \geq 1} T_{0}^{k}
$$

Since for all $k \geq 1$

$$
T_{0}^{k} \geq \theta_{0}\left(\varepsilon_{1}, E_{0}\right) \frac{\varrho_{0}^{2}}{\left(\varrho_{0}^{\frac{1}{2}}+1\right)^{4}}
$$

then by the definition of $T_{0}$, we get

$$
T_{0} \geq \theta_{0}\left(\varepsilon_{1}, E_{0}\right) \frac{\varrho_{0}^{2}}{\left(\varrho_{0}^{\frac{1}{2}}+1\right)^{4}} .
$$

It then follows from the previous analysis that the sequence $\left(\left(v^{k}, d^{k}\right)\right)_{k \in \mathbb{N}}$ is bounded in $C\left(\left[0, T_{0}\right] ; \mathrm{H} \times \mathrm{H}^{1}\right) \cap L^{2}\left(0, T_{0} ; \mathrm{V} \times \mathrm{H}^{2}\right)$ and the sequence $\left(\left(\partial_{t} v^{k}, \partial_{t} d^{k}\right)\right)_{k \in \mathbb{N}}$ is bounded in $L^{2}\left(0, T_{0} ; \mathrm{V}^{*} \times L^{2}\right)$. Hence by Aubin-Lions compactness lemma and BanachAlaoglu theorem, one can extract a subsequence $\left(\left(v^{k_{j}}, d^{k_{j}}\right)\right)_{j \in \mathbb{N}}$ from $\left(\left(v^{k}, d^{k}\right)\right)_{k \in \mathbb{N}}$ and find $(v, d)$ such that as $j \rightarrow \infty$

$$
\begin{aligned}
& \left(v^{k_{j}}, d^{k_{j}}\right) \rightarrow(v, d) \text { weakly in } L^{2}\left(0, T_{0} ; \mathrm{V} \times \mathrm{H}^{2}\right), \\
& \left(v^{k_{j}}, d^{k_{j}}\right) \rightarrow(v, d) \text { weakly star in } L^{\infty}\left(0, T_{0} ; \mathrm{H} \times \mathrm{H}^{1}\right), \\
& \left(v^{k_{j}}, d^{k_{j}}\right) \rightarrow(v, d) \text { strongly in } L^{2}\left(0, T_{0} ; D\left(\mathrm{~A}^{\frac{\theta}{2}}\right) \times \mathrm{H}^{1+\theta}\right) \text { for any } \theta \in[0,1) .
\end{aligned}
$$

Let $t \in\left[0, T_{0}\right]$. Then, the sequence $\left(v^{k}(t), d^{k}(t)\right)_{k \in \mathbb{N}}$ is bounded in $\mathrm{H} \times \mathrm{H}^{1}$. Hence, thanks to the compact embedding $\mathrm{H}^{1} \hookrightarrow L^{2}$ we can and we will assume that the subsequence $\left(\left(v^{k_{j}}, d^{k_{j}}\right)\right)_{j \in \mathbb{N}}$ satisfies, for all $t \in\left[0, T_{0}\right]$

$$
d^{k_{j}}(t) \rightarrow d(t) \text { strongly in } L^{2} .
$$

This and the fact $d^{k_{j}}(t) \in \mathcal{M}$ for all $j \in \mathbb{N}, t \in\left[0, T_{0}\right]$ implies that there exists a constant $C>0$ such that for all $j \in \mathbb{N}, t \in\left[0, T_{0}\right]$

$$
\left.\int_{\Omega}|1-| d(t, x)\right|^{2}\left|\mathrm{~d} x=\int_{\Omega}\right|\left|d^{k_{j}}(t, x)\right|^{2}-|d(t, x)|^{2} \mid \mathrm{d} x
$$




$$
\begin{aligned}
& \leq\left|d^{k_{j}}(t, x)-d(t, x)\right|_{L^{2}}\left|d^{k_{j}}(t, x)+d(t, x)\right|_{L^{2}} \\
& \leq C\left|d^{k_{j}}(t, x)-d(t, x)\right|_{L^{2}} .
\end{aligned}
$$

Passing to the limit as $j \rightarrow \infty$ yields

$$
\left.\int_{\Omega}|1-| d(t, x)\right|^{2} \mid \mathrm{d} x=0, \text { for all } t \in\left[0, T_{0}\right] .
$$

Hence, for all $t \in\left[0, T_{0}\right]$

$$
d(t) \in \mathcal{M}
$$

Our next step is to show that the limit $(v, d)$ satisfies the system (2.13). Hence, we need to pass to the limit in the nonlinear terms. In order to do this, we firstly observe that the convergences

$$
\begin{aligned}
& v^{k_{j}} \cdot \nabla v^{k_{j}} \rightarrow v \cdot \nabla v \text { in } L^{2}\left(0, T_{0} ; \mathrm{V}^{*}\right) \\
& \quad-\operatorname{div}\left(\nabla d^{k_{j}} \odot \nabla d^{k_{j}}\right) \rightarrow-\operatorname{div}(\nabla d \odot \nabla d) \text { in } L^{2}\left(0, T_{0} ; \mathrm{V}^{*}\right) \\
& v^{k_{j}} \cdot \nabla d^{k_{j}} \rightarrow v \cdot \nabla d \text { in } L^{2}\left(0, T_{0} ; L^{2}\right) .
\end{aligned}
$$

are now well known, see, for instance, [37] or [4]; hence, we omit their proof.

Secondly, the most difficult point is the convergence

$$
\left.\lim _{k \rightarrow \infty} \int_{0}^{T_{0}}|| \nabla d^{k}(t)\right|^{2} d^{k}(t)-\left.|\nabla d(t)|^{2} d(t)\right|_{L^{2}} \mathrm{~d} t=0,
$$

and hence we prove it here. For this quest we notice that there exists a constant $C>0$ such that for all $k \geq 1$

$$
\begin{aligned}
\left.\int_{0}^{T_{0}}|| \nabla d^{k}(t)\right|^{2} d^{k}(t)-\left.|\nabla d(t)|^{2} d(t)\right|_{L^{2}} ^{2} \mathrm{~d} t \leq & C \int_{0}^{T_{0}} \mid\left(\nabla d^{k}(t)-\nabla d(t)\right):\left(\nabla d^{k}(t)\right. \\
& +\nabla d(t))\left.d^{k}(t)\right|_{L^{2}} \mathrm{~d} t \\
& +C \int_{0}^{T_{0}}|| \nabla d(t)\left|\left(d^{k}(t)-d(t)\right)\right|_{L^{2}} \mathrm{~d} t .
\end{aligned}
$$

By the Hölder inequality, we have

$$
\begin{aligned}
& \left.\int_{0}^{T_{0}}|| \nabla d^{k}(t)\right|^{2} d^{k}(t)-\left.|\nabla d(t)|^{2} d(t)\right|_{L^{2}} \mathrm{~d} t \\
& \quad \leq C\left(\int_{0}^{T_{0}}\left|\nabla d^{k}(t)-\nabla d(t)\right|_{L^{4}}^{2} \mathrm{~d} t\right)^{\frac{1}{2}}\left(\int_{0}^{T_{0}}\left(\left|\nabla d^{k}(t)\right|_{L^{4}}^{2}+|\nabla d(t)|_{L^{4}}^{2}\right) \mathrm{d} t\right)^{\frac{1}{2}} \sup _{t \in\left[0, T_{0}\right]}\left|d^{k}(t)\right|_{L^{\infty}} \\
& \quad+\left(\int_{0}^{T_{0}}|\nabla d(s)|_{L^{4}}^{2} \mathrm{~d} s\right)^{\frac{1}{2}}\left(\int_{0}^{T_{0}}\left|d^{k}(s)-d(s)\right|_{L^{\infty}}^{2} \mathrm{~d} s\right)^{\frac{1}{2}}
\end{aligned}
$$


By the Ladyzhenskaya inequality ([37, Lemma III.3.3]) and the Sobolev embedding $\mathrm{H}^{1+\theta} \hookrightarrow L^{\infty}(\theta \in(0,1))$, we arrive at

$$
\begin{aligned}
& \left.\int_{0}^{T_{0}}|| \nabla d^{k}(s)\right|^{2} d^{k}(s)-\left.|\nabla d(s)|^{2} d(s)\right|_{L^{2}} \mathrm{~d} s \\
& \leq C\left(\int_{0}^{T_{0}}\left|\nabla d^{k}(s)-\nabla d(s)\right|_{L^{2}}\left|\Delta d^{k}(s)-\Delta d(s)\right|_{L^{2}} \mathrm{~d} s\right)^{\frac{1}{2}} \\
& \quad+C\left(\int_{0}^{T_{0}}\left|d^{k}(s)-d(s)\right|_{\mathrm{H}^{1+\theta}}^{2} \mathrm{~d} s\right)^{\frac{1}{2}} \\
& \quad \leq\left(\int_{0}^{T_{0}}\left|\nabla d^{k}(s)-\nabla d(s)\right|_{L^{2}}^{2} \mathrm{~d} s\right)^{\frac{1}{2}}\left(\int_{0}^{T_{0}}\left|\Delta d^{k}(s)-\Delta d(s)\right|_{L^{2}}^{2} \mathrm{~d} s\right)^{\frac{1}{2}} \\
& \quad+C\left(\int_{0}^{T_{0}}\left|d^{k}(s)-d(s)\right|_{\mathrm{H}^{1+\theta}}^{2} \mathrm{~d} s\right)^{\frac{1}{2}},
\end{aligned}
$$

where we have also used the fact that

$$
\sup _{k} \int_{0}^{T}\left|\nabla d^{k}(s)\right|_{L^{4}}^{2} \mathrm{~d} s<\infty \text { and } d_{k}(t) \in \mathcal{M}, t \in\left[0, T_{0}\right) .
$$

The strong convergence (5.19) and the fact that

$$
\sup _{k}\left(\int_{0}^{T_{0}}\left|\Delta d^{k}(s)-\Delta d(s)\right|_{L^{2}}^{2} \mathrm{~d} s\right)^{\frac{1}{2}}<\infty
$$

completes the proof of (5.21).

We now study the convergence of the term $\phi^{\prime}\left(d^{k}\right)$. Since $d^{k_{j}} \rightarrow \mathrm{d}$ strongly in $L^{2}\left(0, T_{0} ; \mathrm{H}^{1}\right)$, we can assume that $d^{k_{j}} \rightarrow d$ a.e. $(t, x) \in\left[0, T_{0}\right] \times \Omega$. Thus, by the continuity of $\phi^{\prime}($.$) , we get$

$$
\phi^{\prime}\left(d^{k_{j}}\right) \rightarrow \phi^{\prime}(d) \text { a.e. }(t, x) \in\left[0, T_{0}\right) \times \Omega .
$$

Since $d^{k_{j}}(t) \in \mathcal{M}, t \in\left[0, T_{0}\right)$ and, by assumption, $\left|\phi^{\prime}\left(d^{k_{j}}\right)\right| \leq M$, the Lebesgue dominated convergence theorem implies that

$$
\phi^{\prime}\left(d^{k_{j}}\right) \rightarrow \phi^{\prime}(d) \text { in } L^{2}\left(\left[0, T_{0}\right) \times \Omega\right)=L^{2}\left(0, T_{0} ; L^{2}\right) .
$$

Since $d^{k_{j}}(t) \in \mathcal{M}, t \in\left[0, T_{0}\right),\left|\phi^{\prime}\left(d^{k_{j}}\right)\right| \leq M$, we have

$$
\begin{aligned}
& \int_{0}^{T_{0}}\left|\left(d^{k_{j}}(s), \phi^{\prime}\left(d^{k_{j}}(s)\right)\right) d^{k_{j}}(s)-\left(d(s), \phi^{\prime}(d(s))\right) d(s)\right|_{L^{2}}^{2} \mathrm{~d} s \\
& \leq \int_{0}^{T_{0}}\left|\left(d^{k_{j}}(s)-d(s), \phi^{\prime}\left(d^{k_{j}}(s)\right)\right) d^{k_{j}}(s)+\left(d(s), \phi^{\prime}\left(d^{k_{j}}\right)-\phi^{\prime}(d(s))\right) d(s)^{k_{j}}\right|_{L^{2}}^{2} \mathrm{~d} s \\
& \quad+\int_{0}^{T_{0}}\left|\left(d(s), \phi^{\prime}(d)\right)\left(d^{k_{j}}(s)-d(s)\right)\right|_{L^{2}}^{2} \mathrm{~d} s
\end{aligned}
$$




$$
\begin{aligned}
\leq & M \int_{0}^{T_{0}}\left|d^{k_{j}}(s)-d(s)\right|_{L^{2}}^{2} \mathrm{~d} t+\int_{0}^{T_{0}}\left|\phi^{\prime}\left(d^{k_{j}}(s)\right)-\phi^{\prime}(d(s))\right|_{L^{2}}^{2} \mathrm{~d} s \\
& +\int_{0}^{T_{0}}\left|d^{k_{j}}(s)-d(s)\right|_{L^{2}}^{2} \mathrm{~d} s .
\end{aligned}
$$

By the convergence $\phi^{\prime}\left(d^{k_{j}}\right) \rightarrow \phi^{\prime}(d)$ strongly in $L^{2}\left(0, T_{0} ; L^{2}\right)$ and $d^{k_{j}} \rightarrow d$ strongly in $L^{2}\left(0, T_{0} ; L^{\infty}\right)$, we obtain

$$
\alpha\left(d^{k_{j}}\right) d^{k_{j}} \rightarrow \alpha(d) d \text { in } L^{2}\left(0, T_{0} ; L^{2}\right) .
$$

Since

$$
\begin{aligned}
& \int_{0}^{T_{0}}\left|d^{k_{j}}(t) \times g(t)-d(t) \times g(t)\right|_{L^{2}} \mathrm{~d} t \\
& \quad=\int_{0}^{T_{0}}\left|\left(d^{k_{j}}(t)-d(t)\right) \times g(t)\right|_{L^{2}} \mathrm{~d} t \\
& \quad \leq \int_{0}^{T_{0}}\left|d^{k_{j}}(t)-d(t)\right|_{L^{\infty}}|g(t)|_{L^{2}} \mathrm{~d} t \\
& \quad \leq \int_{0}^{T_{0}}\left|d^{k^{j}}(t)-d(t)\right|_{L^{\infty}}^{2} \mathrm{~d} t \int_{0}^{T_{0}}|g(t)|_{L^{2}}^{2} \mathrm{~d} t,
\end{aligned}
$$

and $d^{k_{j}} \rightarrow d$ strongly in $L^{2}\left(0, T_{0} ; L^{\infty}\right)$, we get

$$
d^{k_{j}} \times g \rightarrow d \times g \text { in } L^{1}\left(0, T_{0} ; L^{\infty}\right) .
$$

We will now prove that $(v, d)$ satisfies the initial conditions and that $(v, d) \in$ $C\left(\left[0, T_{0}\right] ; \mathrm{H} \times \mathrm{H}^{1}\right)$. Toward these goals, we first observe that since $(v, d) \in$ $L^{\infty}\left(0, T_{0} ; \mathrm{H} \times \mathrm{H}^{1}\right)$ and

$$
\left(\partial_{t} v^{k_{j}}, \partial_{t} d^{k_{j}}\right) \rightarrow\left(\partial_{t} v, \partial_{t} d\right) \text { in } L^{2}\left(0, T_{0} ; \mathrm{V}^{*} \times L^{2}\right),
$$

then by the Strauss theorem, see [37, Lemma III.1.2], we get

$$
(v, d) \in C\left(\left[0, T_{0}\right] ; \mathrm{H}_{w} \times \mathrm{H}_{w}^{1}\right),
$$

and $d \in C\left(\left[0, T_{0}\right] ; L^{2}\right)$. Hence,

$$
\begin{aligned}
& \lim _{t \rightarrow 0}(v(t), \varphi)=\left(v_{0}, \varphi\right), \forall \varphi \in \mathrm{H}, \\
& \lim _{t \rightarrow 0}(\nabla d(t), \Psi)=\left(\nabla d_{0}, \Psi\right), \forall \Psi \in L^{2}, \\
& \lim _{t \rightarrow 0} \phi(d(t))=\phi\left(d_{0}\right) \text { in } L^{2} .
\end{aligned}
$$

From all these passages to the limits we see that the limit $v$ and $d$ satisfy the equations (3.4) and (3.4) in $\mathrm{V}^{*}$ and $L^{2}$, respectively.

The estimates (5.11) and (5.12) are established by passing to the limit and using the weak lower semicontinuity of the norms in the estimates (5.18), (5.17) and (5.15). 
What remains to prove is the continuity of $(v, d):\left[0, T_{0}\right] \rightarrow \mathrm{H} \times D\left(\hat{\mathrm{A}}^{\frac{1}{2}}\right)$. For this we will firstly establish that

$$
\left(\partial_{t} v, \partial d\right) \in L^{2}\left(0, T ; \mathrm{V}^{*} \times L^{2}\right) .
$$

Toward this aim we recall that it was proved in [4, Proofs of Eqs (3.27), (3.28), (3.31) and (3.32)] that there exists a constant $C>0$ such that for $(v, d) \in C\left(\left[0, T_{0}\right) ; \mathrm{H} \times\right.$ $\left.D\left(\hat{\mathrm{A}}^{\frac{1}{2}}\right)\right) \cap L^{2}\left(0, T_{0} ; \mathrm{V} \times D(\hat{\mathrm{A}})\right)$

$$
\begin{aligned}
|-\mathrm{A} v-B(v, v)-\Pi(\operatorname{div}[\nabla d \odot \nabla d])|_{L^{2}\left(0, T_{0} ; \mathrm{V}^{*}\right)} & \leq C, \\
|-\hat{\mathrm{A}} d-v \cdot \nabla d+d \times g|_{L^{2}\left(0, T_{0} ; L^{2}\right)} & \leq C .
\end{aligned}
$$

Note that the estimates in [4, Eqs (3.27), (3.28), (3.31) and (3.32)] are for $L^{2}\left(0, T_{0} ; \mathrm{V}^{*}\right)$ - and $L^{2}\left(0, T ; L^{2}\right)$-valued random variables, but they remain valid for deterministic $L^{2}\left(0, T_{0} ; \mathrm{V}^{*}\right)$ and $L^{2}\left(0, T ; L^{2}\right)$ functions. Since $d(t) \in \mathcal{M}, t \in\left[0, T_{0}\right)$, we easily infer that there exists a constant $C>0$ such that

$$
\left.\left.|| \nabla d\right|^{2} d\right|_{L^{2}\left(0, T_{0} ; L^{2}\right)} ^{2} \leq|\nabla d|_{L^{4}\left(0, T_{0} ; L^{4}\right)}^{4} \leq \sup _{t \in\left[0, T_{0}\right]}|\nabla d(t)|^{2} \int_{0}^{T_{0}}|d(s)|_{\mathrm{H}^{2}}^{2} \mathrm{~d} s<C .
$$

Using (3.1) and the constraint $d(t) \in \mathcal{M}, t \in\left[0, T_{0}\right.$ ), easily implies that there exists a constant $C>0$ such that

$$
\left|-\phi^{\prime}(d)+\left(\phi^{\prime}(d) \cdot d\right) d\right|_{L^{2}\left(0, T_{0} ; L^{2}\right)} \leq C .
$$

These estimates completes the proof of (5.25).

Secondly, from (5.25), the fact $(v, d) \in L^{2}\left(0, T_{0} ; \mathrm{V} \times D(\hat{\mathrm{A}})\right)$ and [37, Lemma 3.1.2] we infer that

$$
(v, d) \in C\left(\left[0, T_{0}\right] ; \mathrm{H} \times D\left(\hat{\mathrm{A}}^{\frac{1}{2}}\right) .\right.
$$

This completes the proof of Proposition 5.9.

Now, we proceed to the proof of Theorem 5.8.

Proof of Theorem 5.8. In order to prove the theorem, let us denote by $\Sigma$ the set of local solutions to problem (2.13). By Proposition 5.9, the set $\Sigma$ is non-empty and we can and will assume that the time of existence $T_{0}$ of any local solution $(v, d) \in \Sigma$ satisfies the property (5.10) stated in Proposition 5.9. Let us define the relation $\lesssim$ on $\Sigma$ by

$$
\begin{gathered}
\left(y_{1} ; \sigma_{1}\right) \lesssim\left(y_{2} ; \sigma_{2}\right) \text { if } \sigma_{1} \leq \sigma_{2} \text { and } y_{2}=y_{1} \text { on }\left[0, \sigma_{1}\right], \\
\text { for all }\left(y_{i} ; \sigma_{i}\right):=\left(\left(u_{i}, d_{i}\right) ; \sigma_{i}\right) \in \Sigma, i=1,2 .
\end{gathered}
$$

We briefly show below that the relation $\lesssim$ is reflexive, antisymmetric and transitive.

Reflexivity Let $\left(y_{1} ; \sigma_{1}\right) \in \Sigma$. Then $\left(y_{1} ; \sigma_{1}\right) \lesssim\left(y_{1} ; \sigma_{1}\right)$ by definition. 
Antisymmetry Let $\left(y_{i} ; \sigma_{i}\right) \in \Sigma, i=1,2$. Suppose that $\left(y_{1} ; \sigma_{1}\right) \lesssim\left(y_{2} ; \sigma_{2}\right)$ and $\left(y_{2} ; \sigma_{2}\right) \lesssim\left(y_{1} ; \sigma_{1}\right)$. This implies that $y_{2}=y_{1}$ on $\left[0, \sigma_{1}\right], y_{1}=y_{2}$ on $\left[0, \sigma_{2}\right]$ and $\sigma_{1}=\sigma_{2}$. This proves the antisymmetric property of $\lesssim$.

Transitivity Let $\left(y_{i}, \sigma_{i}\right) \in \Sigma, i=1,2,3$. Suppose that $\left(y_{1} ; \sigma_{1}\right) \lesssim\left(y_{2} ; \sigma_{2}\right)$ and $\left(y_{2} ; \sigma_{2}\right) \lesssim\left(y_{3}, \sigma_{3}\right)$. We will prove that $\left(y_{1} ; \sigma_{1}\right) \lesssim\left(y_{3} ; \sigma_{3}\right)$. For this purpose, we observe that

$$
\begin{aligned}
& \sigma_{1} \leq \sigma_{2} \text { and } y_{2}=y_{1} \text { on }\left[0, \sigma_{1}\right] \\
& \sigma_{2} \leq \sigma_{3} \text { and } y_{3}=y_{2} \text { on }\left[0, \sigma_{2}\right] .
\end{aligned}
$$

Hence

$$
\begin{gathered}
\sigma_{1} \leq \sigma_{3}, \\
y_{3}=y_{2}=y_{1} \text { on }\left[0, \sigma_{1}\right] .
\end{gathered}
$$

The facts (5.26) and (5.27) prove the transitivity property of $\lesssim$.

In order to prove the existence of a maximal element in $\Sigma$ we shall use the Kuratowski-Zorn lemma. Hence, we need to prove that all increasing chain in $\Sigma$ has an upper bound. For this purpose, let $\left(\left(v_{k}, d_{k}\right) ; \sigma_{k}\right)_{k \in \mathbb{N}}$ be an increasing chain in $\Sigma$. We will show that this sequence has an upper bound. In order to do that we set

$$
\sigma=\sup _{k \in \mathbb{N}} \sigma_{k}
$$

and define $(v, d):[0, \sigma) \rightarrow \mathrm{H} \times \mathrm{H}^{1}$ by

$$
(v, d)_{\left.\right|_{\left[0, \sigma_{k}\right)}}=\left(v_{k}, d_{k}\right) .
$$

From these definitions it is clear that for all $k \sigma_{k} \leq \sigma$ and $(v, d)=\left(v_{k}, d_{k}\right)$ on $\left[0, \sigma_{k}\right)$ for all $k \in \mathbb{N}$. Hence, $((v, d) ; \sigma) \in \Sigma$ and it is an upper bound of the increasing chain $\left(\left(v_{k}, d_{k}\right) ; \sigma_{k}\right)_{k \in \mathbb{N}}$. Therefore, it now follows from the Kuratowski-Zorn lemma that $\Sigma$ has a maximal element which is a maximal local solution to (2.13). This completes the proof of Theorem 5.8.

The following result gives an important property of the energy of the maximal solution $\left((v, d) ; T_{*}\right)$ near the point $T_{*}$.

Proposition 5.10. Let $\varepsilon_{0}>0, \varrho_{0}>0$ and $\theta_{0}$ be the constants and the function from Proposition 5.9 and

$$
\varepsilon_{1}^{2}=2 \mathcal{E}_{2 \varrho_{0}}\left(v_{0}, d_{0}\right) .
$$

Let $\left((v, d) ; T_{*}\right)$ be the maximal solution defined in Theorem 5.8. Then,

$$
\limsup \sup _{t \nearrow T_{*}} \int_{x \in \Omega}\left[|v(t)|^{2}+|\nabla d(t)|^{2}+\phi(d(t))\right] \mathrm{d} y \geq \varepsilon_{1}^{2},
$$

for all $R \in\left(0, \varrho_{0}\right]$. 
Proof. We prove the proposition by contradiction. Suppose that there exists $R>0$ such that

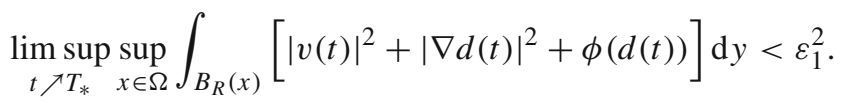

Thus, there exists an increasing sequence $\left(t_{n}\right)_{n \in \mathbb{N}}$ such that

$$
t_{n} \nearrow T_{*} \text { as } n \rightarrow \infty
$$

and

$$
\lim _{n \rightarrow \infty} \sup _{x \in \Omega} \int_{B_{R}(x)}\left[\left|v\left(t_{n}\right)\right|^{2}+\left|\nabla d\left(t_{n}\right)\right|^{2}+\phi\left(d\left(t_{n}\right)\right)\right] \mathrm{d} y<\varepsilon_{1}^{2} .
$$

From (5.29) and (5.30) we deduce that

(a) $\forall \delta>0$, there exists $m \in \mathbb{N}$ such that $0<T_{*}-t_{m}<\delta$,

(b) we can and will assume that for all $n \in \mathbb{N}$

$$
\sup _{x \in \Omega} \int_{B_{R}(x)}\left[\left|v\left(t_{n}\right)\right|^{2}+\left|\nabla d\left(t_{n}\right)\right|^{2}+\phi\left(d\left(t_{n}\right)\right)\right] \mathrm{d} y<\varepsilon_{1}^{2} .
$$

By the global energy inequality (4.9), we get

$$
E_{*}=\sup _{t<T_{*}} \mathcal{E}(v(t), d(t))<\infty .
$$

By (a), for $\delta=\frac{1}{2} \theta_{0}\left(\varepsilon_{1}^{2}, E_{*}\right) R^{2}$, there exists $m \in \mathbb{N}$ such that

$$
0<T_{*}-t_{m}<\frac{1}{2} \theta_{0}\left(\varepsilon_{1}^{2}, E_{*}\right) R_{0}^{2} .
$$

By (b), (5.31) and (5.30) we get

$$
\mathcal{E}\left(v\left(t_{m}\right), d\left(t_{m}\right)\right) \leq E_{*},
$$

and

$$
\sup _{x \in \Omega} \int_{B_{R}(x)}\left[\left|v\left(t_{m}\right)\right|^{2}+\left|\nabla d\left(t_{m}\right)\right|^{2}+\phi\left(d\left(t_{m}\right)\right)\right] \mathrm{d} y<\varepsilon_{1}^{2} .
$$

Hence, by Proposition 5.9, there exists a solution $(\tilde{v}, \tilde{d})$ defined on $\left[t_{m}, \tau+t_{m}\right]$ with

$$
\tau \geq \theta_{0}\left(\varepsilon_{1}^{2}, \mathcal{E}\left(v\left(t_{m}\right), d\left(t_{m}\right)\right) R_{0}^{2}\right.
$$

But observe that $\theta_{0}\left(\varepsilon_{1}^{2}, E_{0}\right)$ is a non-increasing function of the initial energy $E_{0}$. Hence, by (5.32)

$$
\tau \geq \theta_{0}\left(\varepsilon_{1}^{2}, \mathcal{E}\left(v\left(t_{m}\right), d\left(t_{m}\right)\right) R_{0}^{2} \geq \theta_{0}\left(\varepsilon_{1}^{2}, E_{*}\right) R_{0}^{2}>2\left(T_{*}-t_{m}\right) .\right.
$$


By doing elementary calculation we obtain

$$
\begin{aligned}
t_{m}+\tau & \geq 2\left(T_{*}-t_{m}\right)+t_{m} \\
& \geq T_{*}+T_{*}-t_{m} \\
& >T_{*}\left(\text { because } T_{*}-t_{m}>0, \text { see }(5.32)\right) .
\end{aligned}
$$

Hence, we get the existence of a local solution $(\tilde{v}, \tilde{d}):\left[0, \tau+t_{m}\right) \rightarrow \mathrm{H} \times \mathrm{H}^{1}$ with $\tau+t_{m}>T_{*}$ and $(\tilde{v}, \tilde{d})=(v, d)$ on $\left[0, T_{*}\right)$. This contradicts the fact that $\left((v, d) ; T_{*}\right)$ is a maximal solution.

We now give the promised proof of Theorem 5.6.

Proof of Theorem 5.6. Let $\left((v, d), T_{*}\right)$ be the maximal local strong solution to (2.13) constructed from Theorem 5.8. Firstly, we set $T_{*}=T_{1}$ and prove the following result.

Lemma 5.11. The maximal local strong solution $\left((v, d), T_{1}\right)$ satisfies

$$
\begin{array}{r}
\left(\partial_{t} v, \partial_{t} d\right) \in L^{2}\left(0, T_{1} ; D\left(\mathrm{~A}^{-\frac{3}{2}}\right) \times D(\hat{\mathrm{A}})^{*}\right), \\
(v, d) \in C\left(\left[0, T_{1}\right] ; \mathrm{H} \times L^{2}(\Omega)\right) .
\end{array}
$$

Proof of Lemma 5.11. We recall that

$$
\partial_{t} v+A v+\Pi(v \cdot \nabla v)=-\Pi(\operatorname{div}(\nabla d \odot \nabla d))+f .
$$

Firstly, let us prove that $\partial_{t} v \in L^{2}\left(0, T_{1} ; D\left(\mathrm{~A}^{-\frac{3}{2}}\right)\right)$. For this aim, let $\varphi \in D\left(\mathrm{~A}^{\frac{3}{2}}\right)$ be fixed. Then,

$$
\begin{aligned}
\left|\left\langle\partial_{t} v, \varphi\right\rangle\right| & =\left|\int_{\Omega}(\nabla v \cdot \nabla \varphi+v \cdot \nabla v \cdot \varphi) \mathrm{d} x-\int_{\Omega} \nabla d \odot \nabla d: \nabla \varphi \mathrm{d} x+\int_{\Omega} f \varphi \mathrm{d} x\right| \\
& \leq\|\nabla v\|_{L^{2}}\|\nabla \varphi\|_{L^{2}}+\|v\|_{L^{2}}\|\nabla v\|_{L^{2}}\|\varphi\|_{C^{0}(\Omega)}+\|\nabla d\|_{L^{2}}^{2}\|\nabla \varphi\|_{L^{2}}+\|f(t)\|_{\mathrm{H}^{-1}}\|\nabla \varphi\|_{L^{2}} \\
& \leq\left(\|\nabla v\|_{L^{2}}+\|v\|_{L^{2}}\|\nabla v\|_{L^{2}}+\|\nabla d\|_{L^{2}}^{2}+\|f(t)\|_{\mathrm{H}^{-1}}\right)\|\varphi\|_{\mathrm{H}^{3}} \\
& \leq\left(\|\nabla v\|_{L^{2}}+\|v\|_{L^{2}}\|\nabla v\|_{L^{2}}+\|\nabla d\|_{L^{2}}^{2}+\|f(t)\|_{\mathrm{H}^{-1}}\right)\|\varphi\|_{D\left(\mathrm{~A}^{\frac{3}{2}}\right)},
\end{aligned}
$$

where we used the fact $\mathrm{H}^{3}(\Omega) \subset C^{0}(\Omega)$ and $D\left(\mathrm{~A}^{\frac{3}{2}}\right) \hookrightarrow \mathrm{H}^{3}$. Then, we deduce that

$$
\left|\partial_{t} v\right|_{D\left(\mathrm{~A}^{-\frac{3}{2}}\right)} \leq\|\nabla v\|_{L^{2}}+\|v\|_{L^{2}}\|\nabla v\|_{L^{2}}+\|\nabla d\|_{L^{2}}^{2}+\|f\|_{\mathrm{H}^{-1}}
$$

The last line, Assumption 5.4 and the facts $(v, d) \in L^{\infty}\left(0, T_{1} ; \mathrm{H} \times \mathrm{H}^{1}\right)$ and $v \in$ $L^{2}\left(0, T_{1} ; \mathrm{V}\right)$ imply that $\partial_{t} v \in L^{2}\left(0, T_{1} ; D\left(\mathrm{~A}^{-\frac{3}{2}}\right)\right)$. Hence, since $v \in L^{2}\left(0, T_{1} ; \mathrm{V}\right)$ we have $v \in C\left(\left[0, T_{1}\right] ; L^{2}\right)$.

Secondly, we estimate $\partial_{t} d$. For this purpose, let $\Psi \in D(\hat{\mathrm{A}})$ be fixed. Recall that

$$
\partial_{t} d=\Delta d+|\nabla d|^{2} d+v \cdot \nabla d-\phi^{\prime}(d)+\alpha(d) d+d \times g .
$$


Then,

$$
\begin{aligned}
& \left|\left\langle\Delta d+|\nabla d|^{2} d-v \cdot \nabla, \Psi\right\rangle\right| \\
& \quad=\left.\left|\int_{\Omega}(\nabla d \cdot \nabla \Psi+v \cdot \nabla d . \Psi) \mathrm{d} x-\int_{\Omega}\right| \nabla d\right|^{2} d . \Psi \mathrm{d} x \mid \\
& \quad \leq\left(\|\nabla d\|_{L^{2}}+\|v\|_{L^{2}}\|\nabla d\|_{L^{2}}+\|\nabla d\|_{L^{2}}^{2}\right)\|\Psi\|_{\mathrm{H}^{2}} .
\end{aligned}
$$

By the boundedness of $\phi^{\prime}(d)$ on $\mathbb{S}^{2}$, we have

$$
\left|\left\langle-\phi^{\prime}(d)+\alpha(d) d, \Psi\right\rangle\right| \leq C\|\Psi\|_{\mathrm{H}^{2}} .
$$

We also get

$$
|\langle d \times g, \Psi\rangle| \leq C|g(t)|_{L^{2}}\|\Psi\|_{\mathrm{H}^{2}} .
$$

The estimates (5.33)-(5.35) imply that $\partial_{t} d \in L^{2}\left(0, T_{1} ; D(\hat{\mathrm{A}})^{*}\right)$ because $d \in$ $L^{\infty}\left(0, T_{1} ; \mathrm{H}^{1}\right)$ and $v \in L^{\infty}\left(0, T_{1} ; L^{2}\right)$ and $D(\hat{\mathrm{A}}) \hookrightarrow \mathrm{H}^{2}$. The fact $\partial_{t} d \in$ $L^{2}\left(0, T_{1} ; D(\hat{\mathrm{A}})^{*}\right)$ combined with $d \in L^{2}\left(0, T_{1} ; \mathrm{H}^{1}\right)$ imply that $d \in C^{0}\left(\left[0, T_{1}\right] ; L^{2}(\Omega)\right)$, see [37, Lemma III.1.4 ]. This ends the proof of the lemma.

We can now continue with the proof of Theorem 5.8. From Lemma 5.11, we can define

$$
\left(v\left(T_{1}\right), d\left(T_{1}\right)\right)=\lim _{t \nearrow T_{1}}(v(t), d(t)) \text { in } L^{2}(\Omega) \times L^{2}(\Omega)
$$

Since $\nabla d \in L^{\infty}\left(0, T_{1} ; L^{2}(\Omega)\right)$, then $\nabla d(t) \rightarrow \nabla d\left(T_{1}\right)$ weakly in $L^{2}(\Omega)$. This and Theorem D. 1 implies that $v\left(T_{1}\right) \in \mathrm{H}$ and $d\left(T_{1}\right) \in \mathrm{H}^{1}$ and $(v, d) \in C_{w}\left(\left[0, T_{1}\right] ; \mathrm{H} \times\right.$ $\left.\mathrm{H}^{1}\right)$. Moreover, thanks to the strong convergence $d(t) \rightarrow d\left(T_{1}\right)$ in $L^{2}(\Omega)$, we show using the same idea as in the proof of (5.20) that

$$
d\left(T_{1}\right) \in \mathcal{M}
$$

Also, it is not difficult to prove that

$$
\begin{aligned}
\mathcal{E}\left(v\left(T_{1}\right), d\left(T_{1}\right)\right) & \leq \lim _{t \nearrow T_{1}} \mathcal{E}(v(t), d(t)) \\
& \leq \mathcal{E}\left(v_{0}, d_{0}\right)+C\left(|f|_{L^{2}\left(0, T ; \mathrm{H}^{-1}\right)}^{2}+|g|_{L^{2}\left(0, T_{1} ; L^{2}\right)}^{2}\right)<\infty
\end{aligned}
$$

We also prove that

$$
\mathcal{E}\left(v\left(T_{1}\right), d\left(T_{1}\right)\right) \leq \mathcal{E}\left(v_{0}, d_{0}\right)+\frac{1}{2}\left(\int_{0}^{T_{1}}|f(t)|_{\mathrm{H}^{-1}}^{2} \mathrm{~d} t+\int_{0}^{T_{1}}|g(t)|_{L^{2}}^{2} \mathrm{~d} t\right)-\varepsilon_{1}^{2} .
$$


In fact, the inequality (5.44) implies that there exists a sequence $t_{n} \nearrow T_{1}$ and $x_{0} \in \Omega$ such that

$$
\underset{t_{n} \nearrow T_{1}}{\lim \sup _{B_{R}\left(x_{0}\right)}}\left[\left|v\left(t_{n}\right)\right|^{2}+\left|\nabla d\left(t_{n}\right)\right|^{2}+\phi\left(d\left(t_{n}\right)\right)\right] \mathrm{d} x \geq \varepsilon_{1}^{2}, \forall R \in\left(0, \varrho_{0}\right] .
$$

Therefore,

$$
\begin{aligned}
\mathcal{E}\left(v\left(T_{1}\right), d\left(T_{1}\right)\right) & =\lim _{R \searrow 0} \int_{\Omega \backslash B_{R}\left(x_{0}\right)} \mathcal{E}\left(v\left(T_{1}\right), d\left(T_{1}\right)\right) \mathrm{d} y \\
& \leq \lim _{R \searrow 0} \liminf _{t_{n} \nearrow T_{1}} \int_{\Omega \backslash B_{R}\left(x_{0}\right)} \mathcal{E}\left(v\left(t_{n}\right), d\left(t_{n}\right)\right) \mathrm{d} y \\
& \leq \lim _{R \searrow 0}\left[\liminf _{t_{n} \nearrow T_{1}} \int_{\Omega} \mathcal{E}\left(v\left(t_{n}\right), d\left(t_{n}\right)\right) \mathrm{d} y-\limsup _{t_{n} \nearrow T_{1}} \int_{B_{R}\left(x_{0}\right)} \mathcal{E}\left(v\left(t_{n}\right), d\left(t_{n}\right)\right) \mathrm{d} y\right] \\
& \leq \liminf _{t_{n} \nearrow T_{1}} \mathcal{E}\left(v\left(t_{n}\right), d\left(t_{n}\right)\right)-\varepsilon_{1}^{2} \\
& \leq \mathcal{E}\left(v_{0}, d_{0}\right)+\frac{1}{2}\left(\int_{0}^{T_{1}}|f(t)|^{2} \mathrm{~d} t+\int_{0}^{T_{1}}|g(t)|^{2} \mathrm{~d} t\right)-\varepsilon_{1}^{2} .
\end{aligned}
$$

This completes the proof of (5.38).

With (5.37) and (5.36) at hand, one can apply Proposition 5.9 to construct a local strong solution $(\tilde{v}, \tilde{d}):\left[T_{1}, T_{2}\right] \rightarrow \mathrm{H} \times \mathrm{H}^{1}$ with the initial data $\left(\tilde{v}\left(T_{1}\right), \tilde{d}\left(T_{1}\right)\right)=$ $\left(v\left(T_{1}\right), d\left(T_{1}\right)\right) \in \mathrm{H} \times \mathrm{H}^{1}$. Moreover, there are constants $\varrho_{0}>0, \varepsilon_{2} \in\left(0, \varepsilon_{0}\right)$ such that

$$
\limsup \sup _{t \nearrow T_{2}} \int_{x \in \Omega} \mathcal{E}(\tilde{v}(t), \tilde{d}(t)) \geq \varepsilon_{2}^{2}, \forall R \in\left(0, \varrho_{0}\right] .
$$

Furthermore,

$$
\mathcal{E}\left(\tilde{v}\left(T_{2}\right), \tilde{d}\left(T_{2}\right)\right)<\mathcal{E}\left(v\left(T_{1}\right), d\left(T_{1}\right)\right)+\frac{1}{2}\left(\int_{T_{1}}^{T_{2}}|f(t)|_{\mathrm{H}^{-1}}^{2} \mathrm{~d} t+\int_{T_{1}}^{T_{2}}|g(t)|_{L^{2}}^{2} \mathrm{~d} t\right)-\varepsilon_{2}^{2}
$$

Hence, we can construct a sequence of maximal local strong solutions $\left(\left(v_{i}, d_{i}\right) ; T_{i}\right)_{i=1}^{L}$ satisfying: there are constants ${ }^{1} \varrho_{0}>0, \tilde{\varepsilon}_{1} \in\left(0, \varepsilon_{0}\right)$ such that for all $1 \leq i \leq L$

$$
\begin{aligned}
& \limsup _{t \nearrow T_{i}} \sup _{x \in \Omega} \int_{B_{R}(x)} \mathcal{E}\left(v_{i}(t), d_{i}(t)\right) \geq \tilde{\varepsilon}_{1}^{2}, \forall R \in\left(0, \varrho_{0}\right] . \\
& \mathcal{E}\left(v_{i}\left(T_{i}\right), d_{i}\left(T_{i}\right)\right) \leq\left(v_{i}\left(T_{i-1}\right), d_{i}\left(T_{i-1}\right)\right)+\frac{1}{2}\left(\int_{T_{i-1}}^{T_{i}}|f(t)|_{\mathrm{H}^{-1}}^{2} \mathrm{~d} t+\int_{T_{i-1}}^{T_{i}}|g(t)|_{L^{2}}^{2} \mathrm{~d} t\right)-\tilde{\varepsilon}_{1}^{2} .
\end{aligned}
$$

\footnotetext{
${ }^{1}$ In fact, $\tilde{\varepsilon}_{1}=\min \left\{\varepsilon_{i} ; 1 \leq i \leq L\right\}$
} 
In order to construct the global solution we consider a function $(\mathbf{v}, \mathbf{d})$ defined

$$
(\mathbf{v}, \mathbf{d})_{\left.\right|_{\left[T_{i-1}, T_{i}\right)}}=\left(v_{i}, d_{i}\right), \quad 1 \leq i \leq L,
$$

and prove that $L<\infty$. Toward this aim, we first deduce from (5.42) that

$$
\mathcal{E}\left(\mathbf{v}\left(T_{i}\right), \mathbf{d}\left(T_{i}\right)\right) \leq\left(\mathbf{v}\left(T_{i-1}\right), \mathbf{d}\left(T_{i-1}\right)\right)+\frac{1}{2}\left(\int_{T_{i-1}}^{T_{i}}|f(t)|_{\mathrm{H}^{-1}}^{2} \mathrm{~d} t+\int_{T_{i-1}}^{T_{i}}|g(t)|_{L^{2}}^{2} \mathrm{~d} t\right)-\tilde{\varepsilon}_{1}^{2} .
$$

for $i=1, \ldots, L$. Here $T_{0}=0$. Iterating the estimate (5.44) yields

$$
\mathcal{E}\left(\mathbf{v}\left(T_{L}\right), \mathbf{d}\left(T_{L}\right)\right) \leq \mathcal{E}\left(v_{0}, d_{0}\right)+C\left(\int_{0}^{T_{L}}\left(|f(t)|_{\mathrm{H}^{-1}}^{2}+|g(t)|_{L^{2}}^{2}\right) \mathrm{d} t\right)-L \tilde{\varepsilon}_{1}^{2} .
$$

This implies that

$$
L \leq \frac{1}{\tilde{\varepsilon}_{1}^{2}}\left[\mathcal{E}\left(v_{0}, d_{0}\right)+C\left(\int_{0}^{T}\left(|f(t)|_{\mathrm{H}^{-1}}^{2}+|g(t)|_{L^{2}}^{2}\right) \mathrm{d} t\right)\right]<\infty .
$$

In order to complete the proof we need to check that $(\mathbf{v}, \mathbf{d})$ is indeed a global weak solution. But this follows from the definition (5.43), the fact that each $\left(\left(v_{i}, d_{i}\right) ; T_{i}\right)$ are maximal local strong solution defined on $\left[T_{i-1}, T_{i}\right)$ and satisfying

$$
\begin{aligned}
& \left(v_{i}, d_{i}\right) \in C\left(\left[T_{i-1}, T_{i}\right) ; \mathrm{H} \times \mathrm{H}^{1}\right), \\
& \left(\partial_{t} v_{i}, \partial_{t} d_{i}\right) \in L^{2}\left(T_{i-1}, T_{i} ; D\left(\mathrm{~A}^{-\frac{3}{2}}\right) \times D(\hat{\mathrm{A}})^{*}\right), \\
& d_{i}(t) \in \mathcal{M} \text { for all } t \in\left[T_{i-1}, T_{i}\right) .
\end{aligned}
$$

\section{On the regularity and the set of singular times of the solutions when the data are small}

In this section we prove that the set of singular time reduces to the final time horizon $T$ when the data are small enough. Let us start with the following conditional regularity of a strong solution $\left((v, d) ; T_{*}\right)$.

Proposition 6.1. Let $\left(v_{0}, d_{0}\right) \in \mathrm{V} \times D(\hat{\mathrm{A}}), T>0, f \in L^{2}\left(0, T ; L^{2}\right), g \in$ $L^{2}\left(0, T ; \mathrm{H}^{1}\right)$ and $(v, d)$ be a strong solution to $(2.13)$ such that

$$
\begin{aligned}
& v \in C([0, T] ; \mathrm{H}) \cap L^{2}(0, T ; \mathrm{V}) \\
& d \in C\left([0, T] ; \mathrm{H}^{1}\right) \cap L^{2}(0, T ; D(\hat{\mathrm{A}})) .
\end{aligned}
$$


Then,

$$
\begin{aligned}
& v \in C([0, T] ; \mathrm{V}) \cap L^{2}(0, T ; D(\mathrm{~A})) \\
& d \in C([0, T] ; D(\hat{\mathrm{A}})) \cap L^{2}\left(0, T ; D\left(\hat{\mathrm{A}}^{3 / 2}\right)\right) .
\end{aligned}
$$

Proof of Proposition 6.1. We start the proof with the following claim.

Claim 6.2. There exist constants $K>0$ and $K_{7}>0$ depending only on the norms of $\left(v_{0}, d_{0}\right) \in \mathrm{V} \times D\left(\hat{\mathrm{A}}^{\frac{1}{2}}\right)$ and the norms of $(f, g) \in L^{2}\left([0, T] ; \mathrm{H} \times \mathrm{H}^{1}\right)$ such that the following holds. If $(v, d)$ is a regular solution on some interval $\left[0, T_{*}\right] \subset[0, T]$ such that

$$
\int_{0}^{T}|\nabla d(s)|_{L^{4}}^{4} \mathrm{~d} s \leq K_{7} \text { and } \int_{0}^{T}|v(s)|_{L^{4}}^{4} \mathrm{~d} s \leq K_{7},
$$

then,

$$
\begin{gathered}
|\Delta d(t)|_{L^{2}}^{2}+|\nabla v(t)|_{L^{2}}^{2} \leq K, \quad t \in\left[0, T_{*}\right], \\
\int_{0}^{T_{*}}\left(|\mathrm{~A} v(r)|_{L^{2}}^{2}+|\nabla \Delta d(r)|_{L^{2}}^{2}\right) d r \leq K .
\end{gathered}
$$

Proof of claim 6.2. Let $(v, d)$ be a regular solution to (2.13) on $\left[0, T_{*}\right] \subset[0, T]$. Then, we infer from Lemma 4.12 that there exists a constant $K_{3}>0$ which depends on the norms of $\left(v_{0}, d_{0}\right) \in \mathrm{V} \times \mathrm{H}^{1}$ and $(f, g) \in L^{2}\left(0, T ; H \times \mathrm{H}^{1}\right)$ such that

$$
\begin{aligned}
& \sup _{0 \leq s \leq \tau}\left(|\nabla v(s)|_{L^{2}}^{2}+|\Delta d(s)|_{L^{2}}^{2}\right)+2 \int_{0}^{\tau}\left(|\nabla \Delta d(s)|_{L^{2}}^{2}+|A v(s)|_{L^{2}}^{2}\right) \mathrm{d} s \\
& \quad \leq K_{3} e^{K_{3} \int_{0}^{\tau}\left[|\nabla d(r)|_{L^{4}}^{4}+|v(r)|_{L^{4}}^{4}+|\nabla d(r)|_{L^{4}}^{2}+|v(r)|_{L^{4}}^{2}\right] d r .}
\end{aligned}
$$

This and the assumption (6.1) implies the desired inequality (6.2). This proves the claim.

Now we give the proof of Proposition. Since $\left(v_{0}, d_{0}\right) \in \mathrm{V} \times D(\hat{\mathrm{A}}), f \in$ $L^{2}\left(0, T ; L^{2}\right), g \in L^{2}\left(0, T ; H^{1}\right)$, we can apply Theorem 3.7 to assert that there exists a time $T_{0} \leq T$ which depends on $K$ and the norms of $(f, g)$ (on $[0, T]$ ) and a unique solution $(\tilde{v}, \tilde{d}):\left[0, T_{0}\right] \rightarrow \mathrm{V} \times D(\hat{\mathrm{A}})$ to $(2.13)$ such that

$$
\begin{aligned}
& \tilde{v} \in C\left(\left[0, T_{0}\right], \mathrm{V}\right) \cap L^{2}\left(0, T_{0} ; D(\mathrm{~A})\right) \\
& \tilde{d} \in C\left(\left[0, T_{0}\right], D(\hat{\mathrm{A}})\right) \cap L^{2}\left(0, T_{0} ; D\left(\hat{\mathrm{A}}^{3 / 2}\right)\right) .
\end{aligned}
$$

Hence, by Proposition 5.5 we infer that

$$
(\tilde{v}, \tilde{d})=(v, d) \text { on }\left[0, T_{*}\right] .
$$

Thus, since $(v, d)$ is a strong solution on $\left[0, T_{0}\right]$, it follows from the estimates $(5.11)$ and (5.12) that the regular solution $(v, d)$ on $\left[0, T_{0}\right]$ satisfies $(6.1)$ on $\left[0, T_{0}\right]$. Hence, the above claim implies that

$$
(v, d) \in C\left(\left[0, T_{0}\right] ; \mathrm{V} \times D(\hat{\mathrm{A}})\right) \cap L^{2}\left(0, T_{0} ; D(\mathrm{~A}) \times D\left(\hat{\mathrm{A}}^{\frac{3}{2}}\right) .\right.
$$


Thanks to the claim again, we can repeat the above procedure finitely many times, say, on $\left[T_{0}, T_{1}\right], \ldots\left[T_{N}, T_{*}\right]$ to assert that

$$
(v, d) \in C\left(\left[T_{i}, T_{i+1}\right] ; \mathrm{V} \times D(\hat{\mathrm{A}})\right) \cap L^{2}\left(0, T_{0} ; D(\mathrm{~A}) \times D\left(\hat{\mathrm{A}}^{\frac{3}{2}}\right), \quad i \in\{0, \ldots, N\} .\right.
$$

With this we conclude the proof of the proposition.

Theorem 6.3. Let $\varepsilon_{0}>0$ and $\tilde{\varepsilon}_{1} \in\left(0, \varepsilon_{0}\right)$ be the constants from Proposition 5.9 and Theorem 5.6, respectively. If the data $\left(v_{0}, d_{0}\right) \in \mathrm{H} \times \mathrm{H}^{1}$ and $(f, g) \in L^{2}\left(0, T ; L^{2} \times\right.$ $\left.\mathrm{H}^{1}\right)$ satisfy

$$
\mathcal{E}\left(v_{0}, d_{0}\right)+\frac{1}{2} \int_{0}^{T}\left(|f(s)|_{\mathrm{H}^{-1}}^{2}+|g(s)|_{L^{2}}^{2}\right) \mathrm{d} s \leq 2 \tilde{\varepsilon}_{1}^{2},
$$

then (2.13) has a unique global strong solution $(v, d):[0, T) \rightarrow \mathrm{H} \times \mathrm{H}^{1}$ satisfying

$$
(v, d) \in C\left([0, T), \mathrm{H} \times\left(\mathrm{H}^{1} \cap \mathcal{M}\right)\right) \cap C\left([0, T] ; \mathrm{H} \times L^{2}\right) \cap L^{2}(0, T ; \mathrm{V} \times D(\hat{\mathrm{A}})) .
$$

That is, the global strong solution does not have any singular times.

Moreover, if $\left(v_{0}, d_{0}\right) \in V \times D(\hat{\mathrm{A}})$ and $(f, g) \in L^{2}\left(0, T ; \mathrm{H} \times \mathrm{H}^{1}\right)$, then

$$
(v, d) \in C([0, T), \mathrm{V} \times(D(\hat{\mathrm{A}}) \cap \mathcal{M})) \cap L^{2}\left(0, T ; D \mathrm{~A} \times D\left(\hat{\mathrm{A}}^{\frac{3}{2}}\right)\right) .
$$

Proof. Let $\left(v_{0}, d_{0}\right) \in \mathrm{H} \times \mathrm{H}^{1}$ and $(f, g) \in L^{2}\left(0, T ; \mathrm{H}^{-1} \times \mathrm{H}^{1}\right)$. Then, by Theorem 5.6 there exist a finite set $S=\left\{0<T_{1}<\ldots<T_{L}<T\right\}$ and a global weak solution $(v, d)$ to (2.13) satisfying the properties (1)-(3) of Theorem 5.6. We will show that $T_{1}=T$ if the smallness condition (6.4) holds. For this purpose, we argue by contradiction. Assume that $T_{1}<T$ and that $T_{2}=T$. Then, by part (2) of Theorem 5.6

$$
\mathcal{E}\left(v\left(T_{2}\right), d\left(T_{2}\right)\right) \leq \mathcal{E}\left(v_{0}, d_{0}\right)+\frac{1}{2} \int_{0}^{T}\left(|f(s)|_{\mathrm{V}^{*}}^{2}+|g(s)|_{L^{2}}^{2}\right) \mathrm{d} s-2 \tilde{\varepsilon}_{1}^{2},
$$

which altogether with the assumption (6.4) yields

$$
\limsup _{t \rightarrow T_{2}} \sup _{x \in \Omega} \int_{B_{R}(x)}\left[|v(t, y)|^{2}+|\nabla d(t, y)|^{2}+\phi(d(t, y))\right] \mathrm{d} y \leq \mathcal{E}\left(v\left(T_{2}\right), d\left(T_{2}\right)\right) \leq 0 .
$$

This clearly contradicts the fact that

$$
\limsup _{t \rightarrow T_{2}} \sup _{x \in \Omega} \int_{B_{R}(x)}\left[|v(t, y)|^{2}+|\nabla d(t, y)|^{2}+\phi(d(t, y))\right] \mathrm{d} y \geq \tilde{\varepsilon}_{1}^{2}>0,
$$

for all $R \in\left(0, \varrho_{0}\right]$. This completes the proof of the first part of the theorem.

The second part of the theorem follows easily from Proposition 6.1. Hence, the proof of the theorem is completed. 
The last, but not the least, result of this section is about the precompactness of the orbit $(v(t), d(t)), t \in[0, T)$, in $\mathrm{H} \times D(\hat{\mathrm{A}})$. This result will require the following set of conditions on the map $\phi$.

Assumption 6.4. Let $\xi \in \mathbb{R}^{3}$ be a constant vector and $\phi: \mathbb{R}^{3} \rightarrow[0, \infty)$ be the map defined by

$$
\phi(d)=\frac{1}{2}|d-\xi|^{2}, d \in \mathbb{R}^{3} .
$$

It is clear that if $\phi$ satisfies this assumption, then it also satisfies Assumption 3.1.

Theorem 6.5. Let $\varepsilon_{0}>0$ and $\varepsilon_{1} \in\left(0, \varepsilon_{0}\right)$ be the constants from Proposition 5.9 and Theorem 5.6, respectively. Then, there exists a constant $\kappa_{1} \in\left(0, \varepsilon_{1}\right]$ such that the following hold.

$$
\begin{aligned}
& \text { If }\left(v_{0}, d_{0}\right) \in \mathrm{H} \times \mathrm{H}^{1} \text { and }(f, g) \in L^{2}\left(0, T ; \mathrm{V}^{*} \times \mathrm{H}^{1}\right) \text { satisfy } \\
& \qquad \mathcal{E}\left(v_{0}, d_{0}\right)+\frac{1}{2} \int_{0}^{T}\left(|f(s)|_{\mathrm{H}^{-1}}^{2}+|g(s)|_{L^{2}}^{2}\right) \mathrm{d} s \leq 2 \kappa_{1}^{2},
\end{aligned}
$$

then (2.13) has a unique global strong solution $(v, d):[0, T) \rightarrow \mathrm{H} \times \mathrm{H}^{1}$ satisfying:

(1) $(v, d)$ does not have any singular point,

$$
(v, d) \in C([0, T), \mathrm{V} \times D(\hat{\mathrm{A}})) \cap L^{2}\left(0, T ; D(\mathrm{~A}) \times D\left(\hat{\mathrm{A}}^{\frac{3}{2}}\right)\right) .
$$

(2) There exists a constant $K_{1}>0$ such that for all $T>0$

$$
\sup _{t \in[0, T)}\left(|v(t)|_{\mathrm{V}}^{2}+|d(t)|_{D(\hat{\mathrm{A}})}^{2}\right)+\int_{0}^{T}\left(|\mathrm{~A} v(s)|_{L^{2}}^{2}+|\nabla \Delta d(s)|_{L^{2}}^{2}\right) \mathrm{d} s \leq K_{1} .
$$

In particular, the orbit of $(v, d)$ is precompact in $\mathrm{H} \times D\left(\hat{\mathrm{A}}^{\frac{1}{2}}\right)$.

Proof. Let $\left(v_{0}, d_{0}\right) \in \mathrm{V} \times D(\hat{\mathrm{A}}), f \in L^{2}\left(0, T ; L^{2}\right), g \in L^{2}\left(0, T ; \mathrm{H}^{1}\right)$. Let $\varepsilon_{0}>0$ and $\varepsilon_{1} \in\left(0, \varepsilon_{0}\right)$ be the constants from Proposition 5.9 and Theorem 5.6, respectively. Let $\kappa_{0} \in\left(0, \varepsilon_{1}\right]$ be a number to be chosen in (6.18) (see the proof of Proposition 6.6) such that (6.7) holds. Then, by Theorem 6.3 we deduce that the problem (2.13) has a solution $(v, d)$ which does not have singular times and satisfying (6.8). The proof of (6.9) will be proved in Proposition 6.7.

Proposition 6.6. Let $\varepsilon_{0}>0$ and $\varepsilon_{1} \in\left(0, \varepsilon_{0}\right)$ be the constants from Proposition 5.9 and Theorem 5.6, respectively. Then, there exists $\kappa_{1} \in\left(0, \varepsilon_{0}\right)$ and $K_{4}, K_{0}>0$ such that the following hold.

$$
\begin{array}{r}
\text { If }\left(v_{0}, d_{0}\right) \in \mathrm{V} \times D(\hat{\mathrm{A}}), f \in L^{2}\left(0, T ; L^{2}\right), g \in L^{2}\left(0, T ; \mathrm{H}^{1}\right) \text { satisfy } \\
\mathbf{E}_{0}=\mathcal{E}\left(v_{0}, d_{0}\right)+\frac{1}{2} \int_{0}^{\infty}\left[|f(t)|_{\mathrm{H}^{-1}}^{2}+|g(t)|_{L^{2}}^{2}\right] \mathrm{d} t<2 \kappa_{1}^{2},
\end{array}
$$


then any regular solution $(v, d)$ to $(2.13)$ defined on $[0, T)$ satisfies

$$
\begin{aligned}
& \frac{1}{2} \sup _{t \in[0, T)}\left(|v(t)|_{L^{2}}^{2}+|\nabla d(t)|^{2}\right) \\
& +\int_{0}^{T}\left(|\nabla v(r)|_{L^{2}}^{2}+K_{0}|\nabla d(r)|^{2}+K_{0}|\Delta d(r)|_{L^{2}}^{2}\right) d r \leq \mathbf{E}_{0} .
\end{aligned}
$$

Furthermore,

$$
\int_{0}^{T}\left[|\nabla d(t)|_{L^{4}}^{4}+|\nabla d(t)|_{L^{4}}^{2}\right] \mathrm{d} t \leq \frac{K_{4}}{K_{0}}\left(1+\mathbf{E}_{0}^{2}\right)
$$

Proof. Let $\left(v_{0}, d_{0}\right) \in \mathrm{V} \times D(\hat{\mathrm{A}}), f \in L^{2}\left(0, T ; L^{2}\right), g \in L^{2}\left(0, T ; \mathrm{H}^{1}\right)$. Let $\varepsilon_{0}>0$ and $\varepsilon_{1} \in\left(0, \varepsilon_{0}\right)$ be the constants from Proposition 5.9 and Theorem 5.6, respectively. Let $\kappa_{0} \in\left(0, \varepsilon_{1}\right]$ be a number to be chosen later such that $(6.7)$ holds. Let $(v, d)$ be a regular solution to $(2.13)$ defined on $[0, T)$. Then, multiplying the velocity equation by $v$ and using the Cauchy-Schwarz and Young inequalities imply

$$
\frac{1}{2} \frac{\mathrm{d}}{\mathrm{d} t}|v|_{L^{2}}^{2}+\frac{1}{2}|\nabla v|_{L^{2}}^{2} \leq-\langle\Pi \operatorname{div}(\nabla d \odot \nabla d), u\rangle+\frac{1}{2}|f|_{\mathrm{H}^{-1}}^{2} .
$$

We multiply the optical director equation by $-\Delta d$, then use Cauchy-Schwarz and Young inequalities and the constraint $d(t) \in \mathcal{M}, t \in[0, T]$, and obtain

$$
\begin{aligned}
& \frac{1}{2} \frac{\mathrm{d}}{\mathrm{d} t}|\nabla d|_{L^{2}}^{2}+\frac{1}{2}|\Delta d|_{L^{2}}^{2} \\
& \leq\langle v \cdot \nabla d, \Delta d\rangle-\left\langle|\nabla d|^{2} d-\phi^{\prime}(d)+\left(\phi^{\prime}(d) \cdot d\right) d, \Delta d\right\rangle+\frac{1}{2}|g|_{L^{2}}^{2} .
\end{aligned}
$$

Using the fact $\phi^{\prime}(d)=d-\xi$ and the integration-by-parts on the torus we find

$$
\begin{aligned}
& \frac{1}{2} \frac{\mathrm{d}}{\mathrm{d} t}|\nabla d|_{L^{2}}^{2}+\frac{1}{2}|\Delta d|_{L^{2}}^{2}+|\nabla d|_{L^{2}}^{2} \\
& \leq\langle v \cdot \nabla d, \Delta d\rangle+\frac{1}{2}|g|_{L^{2}}^{2}-\left\langle|\nabla d|^{2} d+\left(\phi^{\prime}(d) \cdot d\right) d, \Delta d\right\rangle .
\end{aligned}
$$

Before proceeding further, let us estimate the last term of the above inequality. Toward this end we divide the task into two parts. Firstly, we use fact that $\Delta d \cdot d=-|\nabla d|^{2}$, the Hölder, the Gagliardo-Nirenberg ([1, Section 9.8, Example C.3]), the Young inequalities and [33, Theorem 3.4] to get the following chain of inequalities

$$
\begin{aligned}
\left|\left\langle\left(\phi^{\prime}(d) \cdot d\right) d, \Delta d\right\rangle\right| & =-\int_{\Omega}\left(\phi^{\prime}(d(x)) \cdot d(x)\right)|\nabla d(x)|^{2} \mathrm{~d} x \\
& \leq\left|\phi^{\prime}(d)\right|_{L^{2}}|d|_{L^{\infty}}|\nabla d|_{L^{4}}^{2} \\
& \leq C_{0}|d-\xi|_{L^{2}}\left(|\nabla d|_{L^{2}}\left|\nabla^{2} d\right|_{L^{2}}+|\nabla d|_{L^{2}}^{2}\right) \\
& \leq C_{0}|d-\xi|_{L^{2}}\left(|\nabla d|_{L^{2}}|\Delta d|_{L^{2}}+|\nabla d|_{L^{2}}^{2}\right)
\end{aligned}
$$




$$
\leq \frac{1}{2}|\nabla d|_{L^{2}}^{2}+2 C_{0}^{2} \frac{1}{2}|d-\xi|_{L^{2}}^{2}\left(|\Delta d|_{L^{2}}^{2}+|\nabla d|_{L^{2}}^{2}\right) .
$$

Secondly, using the Gagliardo-Nirenberg inequality ([1, Section 9.8, Example C.3]) and [33, Theorem 3.4] we obtain

$$
\begin{aligned}
-\left\langle|\nabla d|^{2} d, \Delta d\right\rangle & =|\nabla d|_{L^{4}}^{4} \\
& \leq C_{1}|\nabla d|_{L^{2}}^{2}\left(|\Delta d|_{L^{2}}^{2}+|\nabla d|_{L^{2}}^{2} .\right.
\end{aligned}
$$

Plugging (6.14) and (6.15) into the inequality (6.13) yields

$$
\begin{aligned}
& \frac{1}{2} \frac{\mathrm{d}}{\mathrm{d} t}|\nabla d|_{L^{2}}^{2}+|\nabla d|_{L^{2}}^{2} \\
& \quad \leq\langle v \cdot \nabla d, \Delta d\rangle+\frac{1}{2}|g|_{L^{2}}^{2}-\left(\frac{1}{2}-2 C_{0}^{2} \frac{1}{2}|d-\xi|_{L^{2}}^{2}-C_{1}|\nabla d|_{L^{2}}^{2}\right)|\Delta d|_{L^{2}}^{2} \\
& \quad-\left(\frac{1}{2}-2 C_{0}^{2} \frac{1}{2}|d-\xi|_{L^{2}}^{2}-C_{1}|\nabla d|_{L^{2}}^{2}\right)|\nabla d|_{L^{2}}^{2}
\end{aligned}
$$

Now adding the inequalities (6.12) and (6.16) side by side, and using (4.8) imply

$$
\begin{aligned}
\frac{1}{2} \frac{\mathrm{d}}{\mathrm{d} t}\left(|\nabla d|_{L^{2}}^{2}+|v|_{L^{2}}^{2}\right) \frac{1}{2}|\nabla v|_{L^{2}}^{2} \leq & -\left(\frac{1}{2}-2 C_{0}^{2} \frac{1}{2}|d-\xi|_{L^{2}}^{2}-C_{1}|\nabla d|_{L^{2}}^{2}\right)|\Delta d|_{L^{2}}^{2} \\
& -\left(\frac{1}{2}-2 C_{0}^{2} \frac{1}{2}|d-\xi|_{L^{2}}^{2}-C_{1}|\nabla d|_{L^{2}}^{2}\right)|\nabla d|_{L^{2}}^{2} \\
& +\frac{1}{2}\left(|g|_{L^{2}}^{2}+|f|_{\mathrm{H}^{-1}}\right) .
\end{aligned}
$$

Thanks to the energy inequality (4.9) we obtain

$$
\begin{aligned}
& \frac{1}{2} \frac{\mathrm{d}}{\mathrm{d} t}\left(|\nabla d|_{L^{2}}^{2}+|v|_{L^{2}}^{2}\right)+|\nabla d|_{L^{2}}^{2}+\frac{1}{2}|\nabla v|_{L^{2}}^{2} \leq-\left(\frac{1}{2}-2 C_{0}^{2} \mathbf{E}_{0}-C_{1} \mathbf{E}_{0}\right)|\Delta d|_{L^{2}}^{2} \\
& \quad-\left(\frac{1}{2}-2 C_{0}^{2} \mathbf{E}_{0}-C_{1} \mathbf{E}_{0}\right)|\nabla d|_{L^{2}}^{2}+\frac{1}{2}\left(|g|_{L^{2}}^{2}+|f|_{\mathrm{H}^{-1}}\right) .
\end{aligned}
$$

We now easily conclude the proof of (6.10) in the proposition by integrating (6.17), taking the supremum over $t \in\left[0, T_{0}\right]$ and choosing

$$
\kappa_{1}^{2}=\min \left\{\varepsilon_{1}^{2}, \frac{1}{4\left(C_{0}^{2}|\Omega|+C_{1}\right)}\right\} \text { and } K_{0}=\frac{1}{2}-\mathbf{E}_{0}\left(C_{0}^{2}|\Omega|+C_{1}\right) .
$$

In order to prove the second estimate, we use the Gagliardo-Nirenberg inequality (see [1, Section 9.8, Example C.3]) and (6.10) to obtain

$$
\begin{aligned}
\int_{0}^{T}\left(|\nabla d|_{L^{4}}^{4}+\left.|| \nabla d\right|_{L^{4}} ^{2}\right) \mathrm{d} t & \leq C \sup _{t \in[0, T]}|\nabla d(t)|_{L^{2}}^{2} \int_{0}^{T}|\Delta d(t)|^{2} \mathrm{~d} t+C \int_{0}^{T}|\nabla d(s)|_{L^{2}}^{2} \mathrm{~d} s \\
& +C \int_{0}^{T}\left(|\nabla d(s)|^{2}+|\Delta d(s)|_{L^{2}}^{2} \mathrm{~d} s\right)
\end{aligned}
$$




$$
\leq \frac{C}{K_{0}}\left[\mathbf{E}_{0}^{2}+\mathbf{E}_{0}\right]
$$

This completes the proof of the proposition.

The next result is crucial for the proof of Theorem 6.5.

Proposition 6.7. Let $\varepsilon_{0}$ be as in Proposition 5.9 and $\left(v_{0}, d_{0}\right) \in \mathrm{V} \times D(\hat{\mathrm{A}}), f \in$ $L^{2}\left(0, T ; L^{2}\right), g \in L^{2}\left(0, T ; H^{1}\right)$. Assume that

$$
\mathbf{E}_{0}=\mathcal{E}\left(v_{0}, d_{0}\right)+\frac{1}{2} \int_{0}^{\infty}\left[|f(t)|_{\mathrm{H}^{-1}}^{2}+|g(t)|_{L^{2}}^{2}\right] \mathrm{d} t<\kappa_{1}^{2},
$$

where $\kappa_{1} \in\left(0, \varepsilon_{0}\right)$ is defined in Proposition 6.6. Then, there exist a constant $K_{2}>0$, which depends only on the norms of $\left(v_{0}, d_{0}\right) \in \mathrm{V} \times D(\hat{\mathrm{A}})$ and $(f, g) \in L^{2}(0, T ; \mathrm{H} \times$ $\left.\mathrm{H}^{1}\right)$, such that for a regular solution $(v, d)$ to $(2.13)$ we have

$$
\begin{aligned}
& \frac{1}{2} \sup _{t \in[0, T)}\left(\left|\mathrm{A}^{\frac{1}{2}} v(t)\right|_{L^{2}}^{2}+|\Delta d(t)|^{2}\right) \\
& \quad+\int_{0}^{T_{*}}\left(|\mathrm{~A} v(r)|_{L^{2}}^{2}+|\Delta d(r)|^{2}+|\Delta d(r)|_{L^{2}}^{2}\right) d r \leq K_{2} .
\end{aligned}
$$

Proof. The proof of the estimate (6.19) is very similar to the proof of (4.22); hence, we only sketch the proof. We start with the same idea as in the proof of (4.22), i.e., we multiply the velocity and optical director equations by $A v$ and $\mathrm{A}^{2} d$, respectively. This procedure implies the equation (4.23). In the present proof, we estimate the term $\mathrm{H}^{1}\left\langle\phi^{\prime}(d),-\hat{\mathrm{A}}^{2} d\right\rangle_{\left(\mathrm{H}^{1}\right)^{*}}$ as follows:

$$
\mathrm{H}^{1}\left\langle\phi^{\prime}(d),-\hat{\mathrm{A}}^{2} d\right\rangle_{\left(\mathrm{H}^{1}\right)^{*}}=-\left\langle\Delta \phi^{\prime}(d), \Delta d\right\rangle=-|\Delta d|_{L^{2}}^{2} .
$$

This gives raise to the term $\int_{0}^{T}|\Delta d(r)|^{2} d r$ in the left hand side of (6.19). Now, the remaining terms are estimated with exactly the same way as in the proof of (4.23). In particular, we infer that there exist constants $K_{3}>0$, which depends on the norms of $\left(u_{0}, d_{0}\right) \in \mathrm{V} \times D(\hat{\mathrm{A}})$ and $(f, g) \in L^{2}\left(0, T ; \mathrm{H} \times D\left(\hat{\mathrm{A}}^{1 / 2}\right)\right)$, and $K_{4}>0$ such that for all $\tau \in[0, T)$ we have

$$
\begin{aligned}
& \sup _{0 \leq s \leq \tau}\left(|\nabla v(s)|_{L^{2}}^{2}+|\Delta d(s)|_{L^{2}}^{2}\right)+2 \int_{0}^{\tau}\left(|\nabla \Delta d(s)|_{L^{2}}^{2}+|\Delta d(s)|_{L^{2}}^{2}+|A v(s)|_{L^{2}}^{2}\right) \mathrm{d} s \\
& \quad \leq K_{3} e^{K_{4} \int_{0}^{\tau}\left[|\nabla d(r)|_{L^{4}}^{4}+|v(r)|_{L^{4}}^{4}+|\nabla d(r)|_{L^{4}}^{2}+|v(r)|_{L^{4}}^{2}\right] d r} .
\end{aligned}
$$

Since $L^{4} \cap \mathrm{H} \subset \mathrm{V}$, by using (6.10) and the Ladyzhenskaya inequality ([37, Lemma III.3.3]) we infer that there exists $C>0$ such that

$$
\int_{0}^{T}\left[|v(s)|_{L^{4}}^{4}+|v(s)|_{L^{4}}^{2}\right] \mathrm{d} s \leq \int_{0}^{T}\left[|v(s)|_{L^{4}}^{4}+|\nabla v(s)|^{2}\right] \mathrm{d} s \leq C\left(1+\mathbf{E}_{0}^{2}\right) .
$$

Thus, plugging the latter estimate and (6.11) into (6.20) imply (6.19). This completes the proof of the proposition. 
Open Access. This article is licensed under a Creative Commons Attribution 4.0 International License, which permits use, sharing, adaptation, distribution and reproduction in any medium or format, as long as you give appropriate credit to the original author(s) and the source, provide a link to the Creative Commons licence, and indicate if changes were made. The images or other third party material in this article are included in the article's Creative Commons licence, unless indicated otherwise in a credit line to the material. If material is not included in the article's Creative Commons licence and your intended use is not permitted by statutory regulation or exceeds the permitted use, you will need to obtain permission directly from the copyright holder. To view a copy of this licence, visit http://creativecommons.org/licenses/ by/4.0/.

Publisher's Note Springer Nature remains neutral with regard to jurisdictional claims in published maps and institutional affiliations.

\section{Appendix A: Proof of Lemma 3.9}

In this section we will prove Lemma 3.9.

Proof of Lemma 3.9. Firstly, we infer from [5, Lemma 2.5 ${ }^{2}$ that there exists a constant $C>0$ such that for all $\left(v_{i}, d_{i}\right) \in D(\mathrm{~A}) \times D\left(\hat{\mathrm{A}}^{\frac{3}{2}}\right)$

$$
\begin{aligned}
& \left|F\left(v_{1}, n_{1}\right)-F\left(v_{2}, n_{2}\right)\right|_{\mathrm{H}} \leq C\left|\mathrm{~A}^{\frac{1}{2}}\left[v_{1}-v_{2}\right]\right|_{L^{2}}^{\frac{1}{2}}\left(\left|\mathrm{~A}\left[v_{1}-v_{2}\right]\right|_{L^{2}}^{\frac{1}{2}}\left|\mathrm{~A}^{\frac{1}{2}} u_{2}\right|_{L^{2}}\right. \\
& \left.\quad+\left|\mathrm{A}^{\frac{1}{2}}\left[v_{1}-v_{2}\right]\right|_{L^{2}}^{\frac{1}{2}}\left|\mathrm{~A}^{\frac{1}{2}} u_{1}\right|_{L^{2}}^{\frac{1}{2}}\left|\mathrm{~A} u_{1}\right|_{L^{2}}^{\frac{1}{2}}\right) \\
& \quad+\left|n_{1}-n_{2}\right|_{\mathrm{H}^{2}}\left|n_{1}\right|_{\mathrm{H}^{2}}^{\frac{1}{2}}\left|n_{1}\right|_{\mathrm{H}^{3}}^{\frac{1}{2}}+\left|n_{1}-n_{2}\right|_{\mathrm{H}^{2}}^{\frac{1}{2}}\left|n_{1}-n_{2}\right|_{\mathrm{H}^{3}}^{\frac{1}{2}}\left|n_{2}\right|_{\mathrm{H}^{2}}^{\frac{1}{2}}
\end{aligned}
$$

This clearly implies that there exists a constant $C>0$ such that for all $\left(v_{i}, d_{i}\right) \in \mathbf{X}_{T}$, $i=1,2$,

$$
\begin{aligned}
&\left|F\left(v_{1}, n_{1}\right)-F\left(v_{2}, n_{2}\right)\right|_{L^{2}(0, T ; \mathrm{H})}^{2} \\
& \leq C T^{\frac{1}{2}}\left|v_{1}-v_{2}\right|_{C([0, T] ; \mathrm{V})}\left|u_{2}\right| C([0, T] ; \mathrm{V})\left|\mathrm{A}\left[v_{1}-v_{2}\right]\right|_{L^{2}(0, T ; D(\mathrm{~A}))} \\
&+C T^{\frac{1}{2}}\left|\mathrm{~A}^{\frac{1}{2}}\left[v_{1}-v_{2}\right]\right|_{C([0, T] ; \mathrm{V})}^{2}\left|u_{1}\right|_{C([0, T] ; \mathrm{V})}\left|\mathrm{A} u_{1}\right|_{L^{2}(0, T ; D(\mathrm{~A}))} \\
&+C T^{\frac{1}{2}}\left|n_{1}-n_{2}\right|_{C\left([0, T] ; \mathrm{H}^{2}\right)}^{2}\left|n_{1}\right|_{C\left([0, T] ; \mathrm{H}^{2}\right)}\left|n_{1}\right|_{L^{2}\left(0, T ; \mathrm{H}^{3}\right)} \\
&+C T^{\frac{1}{2}}\left|n_{1}-n_{2}\right|_{C\left([0, T] ; \mathrm{H}^{2}\right)}\left|n_{1}-n_{2}\right|_{L^{2}\left(0, T ; \mathrm{H}^{3}\right)}\left|n_{2}\right|_{C\left([0, T] ; \mathrm{H}^{2}\right)} .
\end{aligned}
$$

Hence, we infer that there exists a constant $C>0$ such that for all $\left(v_{i}, d_{i}\right) \in \mathbf{X}_{T}$, $i=1,2$,

$$
\begin{array}{r}
\left|F\left(v_{1}, n_{1}\right)-F\left(v_{2}, n_{2}\right)\right|_{L^{2}(0, T ; \mathrm{H})}^{2} \leq C T^{\frac{1}{2}}\left|v_{1}-v_{2}\right|_{\mathbf{X}_{T}^{1}}^{2}\left[\left|u_{1}\right|_{\mathbf{X}_{T}^{1}}^{2}+\left|u_{2}\right|_{\mathbf{X}_{T}^{1}}^{2}\right] \\
C T^{\frac{1}{2}}\left|n_{1}-n_{2}\right|_{\mathbf{X}_{T}^{1}}^{2}\left[\left|d_{1}\right|_{\mathbf{X}_{T}^{2}}^{2}+\left|d_{2}\right|_{\mathbf{X}_{T}^{2}}^{2}\right],
\end{array}
$$

from which we easily deduce the inequality (3.11).

\footnotetext{
2 Note that in [5, Lemma 2.5] the authors used $B_{1}\left(v_{i}, v_{i}\right)$ and $M\left(n_{i}, n_{i}\right)$ in places of $B\left(v_{i}, v_{i}\right)$ and $\Pi\left(\nabla n_{i} \Delta n_{i}\right)$, respectively.
} 
We will now proceed to the proof of (3.12) which will be divided into four steps. Firstly, because of the assumption (3.3) the map $\phi^{\prime}: \mathbb{R}^{3} \rightarrow \mathbb{R}^{3}$ is Lipschitz continuous. Hence, for all $n_{i} \in \mathrm{H}^{2}, i=1,2$,

$$
\begin{aligned}
\left|\phi^{\prime}\left(n_{1}\right)-\phi^{\prime}\left(n_{2}\right)\right|_{\mathrm{H}^{1}} & =\left|\phi^{\prime}\left(n_{1}\right)-\phi^{\prime}\left(n_{2}\right)\right|_{L^{2}}+\left|\nabla \phi^{\prime}\left(n_{1}\right)-\nabla \phi^{\prime}\left(n_{2}\right)\right|_{L^{2}} \\
& \leq M_{2}\left|n_{1}-n_{2}\right|_{L^{2}}+\left|\phi^{\prime \prime}\left(n_{1}\right) \nabla n_{1}-\phi^{\prime \prime}\left(n_{2}\right) \nabla n_{2}\right|_{L^{2}} \\
& \leq M_{2}\left|n_{1}-n_{2}\right|_{L^{2}}+\left|\left[\phi^{\prime \prime}\left(n_{1}\right)-\phi^{\prime \prime}\left(n_{2}\right)\right] \nabla n_{1}+\phi^{\prime \prime}\left(n_{2}\right) \nabla\left(n_{1}-n_{2}\right)\right|_{L^{2}} .
\end{aligned}
$$

Using (3.2), (3.3) and the Sobolev embedding $\mathrm{H}^{1} \subset L^{4}$ we obtain

$$
\begin{aligned}
\left|\phi^{\prime}\left(n_{1}\right)-\phi^{\prime}\left(n_{2}\right)\right|_{\mathrm{H}^{1}} & \leq M_{2}\left|n_{1}-n_{2}\right|_{L^{2}}+|| n_{1}-n_{2}|| \nabla n_{1}||_{L^{2}}+M_{2}\left|\nabla\left(n_{1}-n_{2}\right)\right|_{L^{2}} \\
& \leq M_{2}\left|n_{1}-n_{2}\right|_{\mathrm{H}^{1}}+\left|n_{1}-n_{2}\right|_{L^{4}}\left|\nabla n_{1}\right|_{L^{4}} \\
& \leq M_{2}\left|n_{1}-n_{2}\right|_{\mathrm{H}^{1}}+\left|n_{1}-n_{2}\right|_{\mathrm{H}^{1}}\left|n_{1}\right|_{\mathrm{H}^{2}} .
\end{aligned}
$$

Hence, there exists a constant $C>0$ such that for all $n_{i} \in \mathbf{X}_{T}^{2}, i=1,2$

$$
\left|\phi^{\prime}\left(n_{1}\right)-\phi^{\prime}\left(n_{2}\right)\right|_{L^{2}\left(0, T ; \mathrm{H}^{1}\right)}^{2} \leq M_{2} T\left|n_{1}-n_{2}\right|_{\mathbf{X}_{T}^{2}}^{2}\left[1+\left|n_{1}\right|_{\mathbf{X}_{T}^{2}}^{2}\right] .
$$

Secondly, we estimate the term involving $\left(\mid\left(\phi^{\prime}\left(n_{i}\right) \cdot n_{i}\right) n_{i}\right), i=1,2$, as follows. Let $n_{i} \in \mathrm{H}^{2}, i=1,2$. Then, we have

$$
\begin{aligned}
& \left|\left(\phi^{\prime}\left(n_{1}\right) \cdot n_{1}\right) n_{1}-\left(\phi^{\prime}\left(n_{2}\right) \cdot n_{2}\right) n_{2}\right|_{\mathrm{H}^{1}} \\
& \quad=\left|\left(\left[\phi^{\prime}\left(n_{1}\right)-\phi^{\prime}\left(n_{2}\right)\right] \cdot n_{1}\right) n_{1}+\left(\phi^{\prime}\left(n_{2}\right) \cdot n_{1}\right) n_{1}-\left(\phi^{\prime}\left(n_{2}\right) \cdot n_{2}\right) n_{2}\right|_{\mathrm{H}^{1}} \\
& \quad \leq\left|\left(\left[\phi^{\prime}\left(n_{1}\right)-\phi^{\prime}\left(n_{2}\right)\right] \cdot n_{1}\right) n_{1}\right|_{\mathrm{H}^{1}}+\left|\left(\phi^{\prime}\left(n_{2}\right) \cdot\left[n_{1}-n_{2}\right]\right) n_{1}\right|_{\mathrm{H}^{1}} \\
& \quad+\left|\left(\phi^{\prime}\left(n_{2}\right) \cdot n_{2}\right)\left[n_{1}-n_{2}\right]\right|_{\mathrm{H}^{1}} .
\end{aligned}
$$

We recall the following classical fact whose proof is easy and omitted.

$$
|f g|_{\mathrm{H}^{1}} \leq 2\left(|f|_{L^{\infty}}|g|_{\mathrm{H}^{1}}+|f|_{\mathrm{H}^{1}}|g|_{L^{\infty}}\right), \text { for all } f, g \in \mathrm{H}^{1} \cap L^{\infty} \text {. }
$$

Since, $n_{i} \in \mathrm{H}^{2}$ and $\mathrm{H}^{2} \subset L^{\infty}$, it follows from (3.1) and (3.3) that $\phi^{\prime}\left(n_{i}\right) \in \mathrm{H}^{1} \cap L^{\infty}$. Hence, we can repeatedly use (A.2), the Lipschitz property of $\phi^{\prime}$ to infer that for all $n_{i} \in \mathrm{H}^{2}, i=1,2$,

$$
\begin{aligned}
& \left|\left(\left[\phi^{\prime}\left(n_{1}\right)-\phi^{\prime}\left(n_{2}\right)\right] \cdot n_{1}\right) n_{1}\right|_{\mathrm{H}^{1}} \leq C\left|\phi^{\prime}\left(n_{1}\right)-\phi^{\prime}\left(n_{2}\right)\right|_{L^{\infty}}\left|n_{1}\right|_{L^{\infty}}\left|n_{1}\right|_{\mathrm{H}^{1}} \\
& \quad+\left|\phi^{\prime}\left(n_{1}\right)-\phi^{\prime}\left(n_{2}\right)\right|_{\mathrm{H}^{1}}\left|n_{1}\right|_{L^{\infty}}^{2} . \\
& \quad \leq\left|n_{1}-n_{2}\right|_{L^{\infty}}\left|n_{1}\right|_{\mathrm{H}^{2}}^{2}+\left|\phi^{\prime}\left(n_{1}\right)-\phi^{\prime}\left(n_{2}\right)\right|_{\mathrm{H}^{1}}\left|n_{1}\right|_{\mathrm{H}^{2}}^{2} \\
& \quad \leq\left|n_{1}-n_{2}\right|_{\mathrm{H}^{2}}\left|n_{1}\right|_{\mathrm{H}^{2}}^{2}+\left|\phi^{\prime}\left(n_{1}\right)-\phi^{\prime}\left(n_{2}\right)\right|_{\mathrm{H}^{1}}\left|n_{1}\right|_{\mathrm{H}^{2}}^{2} .
\end{aligned}
$$

Thanks to the last line and (A.1) we deduce that there exists $C>0$ such that for all $n_{1}, n_{2} \in \mathbf{X}_{T}^{2}$

$$
\left|\left(\left[\phi^{\prime}\left(n_{1}\right)-\phi^{\prime}\left(n_{2}\right)\right] \cdot n_{1}\right) n_{1}\right|_{L^{2}\left(0, T ; \mathrm{H}^{1}\right)}^{2} \leq C T\left|n_{1}-n_{2}\right|_{\mathbf{X}_{T}^{2}}^{2}\left|n_{1}\right|_{\mathbf{X}_{T}^{2}}^{4}\left[1+\left|n_{1}\right|_{\mathbf{X}_{T}^{2}}^{2}\right],
$$


Using (A.2) we infer that there exists a constant $C>0$ such that for all $a, b, c \in \mathrm{H}^{2}$ $\left|\left(\phi^{\prime}(a) \cdot b\right) c\right|_{\mathrm{H}^{1}} \leq C\left|\phi^{\prime}(a)\right|_{L^{\infty}}\left[|b|_{L^{\infty}}|c|_{\mathrm{H}^{1}}+|b|_{\mathrm{H}^{1}}|c|_{L^{\infty}}\right]+\left|\phi^{\prime}(a)\right|_{\mathrm{H}^{1}}|b|_{L^{\infty}}|a|_{L^{\infty}}$.

This altogether with (3.1), (3.3) and the continuous Sobolev embeddings $\mathrm{H}^{2} \hookrightarrow$ $L^{\infty}$ and $\mathrm{H}^{2} \subset \mathrm{H}^{1}$ implies that for all $a, b, c \in \mathrm{H}^{2}$

$$
\left|\left(\phi^{\prime}(a) \cdot b\right) c\right|_{\mathrm{H}^{1}} \leq C\left(1+|a|_{\mathrm{H}^{2}}\right)|b|_{\mathrm{H}^{2}}|c|_{\mathrm{H}^{2}},
$$

Thus, there exists a constant $C>0$ such that $n_{1}, n_{2} \in \mathrm{H}^{2}$

$$
\begin{aligned}
& \left|\left(\phi^{\prime}\left(n_{2}\right) \cdot\left[n_{1}-n_{2}\right]\right) n_{1}\right|_{\mathrm{H}^{1}} \leq C\left(1+\left|n_{2}\right|_{\mathrm{H}^{2}}\right)\left|n_{1}-n_{2}\right|_{\mathrm{H}^{2}}\left|n_{1}\right|_{\mathrm{H}^{2}} \\
& \mid\left(\phi^{\prime}\left(n_{2}\right) \cdot n_{2}\right)\left[n_{1}-\left.n_{2}\right|_{\mathrm{H}^{1}} \leq C\left(1+\left|n_{2}\right|_{\mathrm{H}^{2}}\right)\left|n_{2}\right|_{\mathrm{H}^{2}}\left|n_{1}-n_{2}\right|_{\mathrm{H}^{2}} .\right.
\end{aligned}
$$

Thus, we deduce that there exists constant $C>0$ such that for all $n_{1}, n_{2} \in \mathbf{X}_{T}^{2}$

$$
\begin{aligned}
& \left|\left(\phi^{\prime}\left(n_{2}\right) \cdot\left[n_{1}-n_{2}\right]\right) n_{1}\right|_{L^{2}\left(0, T ; \mathrm{H}^{1}\right)}^{2} \leq C T\left(1+\left|n_{2}\right|_{\left.\mathbf{X}_{T}^{2}\right)}^{2}\left|n_{1}-n_{2}\right|_{\mathbf{X}_{T}^{2}}^{2}\left|n_{1}\right|_{\mathbf{X}_{T}^{2}}^{2}\right. \\
& \left|\left(\phi^{\prime}\left(n_{2}\right) \cdot n_{2}\right)\left[n_{1}-n_{2}\right]\right|_{L^{2}\left(0, T ; \mathrm{H}^{1}\right)}^{2} \leq C T\left(1+\left|n_{2}\right|_{\mathbf{X}_{T}^{2}}^{2}\right)\left|n_{2}\right|_{\mathbf{X}_{T}^{2}}^{2}\left|n_{1}-n_{2}\right|_{\mathbf{X}_{T}^{2}}^{2} .
\end{aligned}
$$

Therefore, there exists a constant $C>0$ such that for all $n_{i} \in \mathbf{X}^{2} T, i=1,2$,

$$
\left|\left(\left[\phi^{\prime}\left(n_{1}\right)-\phi^{\prime}\left(n_{2}\right)\right] \cdot n_{1}\right) n_{1}\right|_{L^{2}\left(0, T ; \mathrm{H}^{1}\right)} \leq C T\left|n_{1}-n_{2}\right|_{\mathbf{X}_{T}^{2}}^{2}\left(1+\left|n_{1}\right|_{\mathbf{X}_{T}^{2}}^{4}+\left|n_{1}\right|_{\mathbf{X}_{T}^{2}}^{6}+\left|n_{2}\right|_{\mathbf{X}_{T}^{2}}^{2}\right) .
$$

From (A.3)-(A.5) we infer that there exists a constant $C>0$ such that for all $n_{i} \in \mathbf{X}_{T}^{2}$, $i=1,2$,

$$
\left|\left(\phi^{\prime}\left(n_{1}\right) \cdot n_{1}\right) n_{1}-\left(\phi^{\prime}\left(n_{2}\right) \cdot n_{2}\right) n_{2}\right|_{L^{2}\left(0, T ; \mathrm{H}^{1}\right)} \leq C T\left|n_{1}-n_{2}\right|_{\mathbf{X}_{T}^{2}}^{2}\left(1+\sum_{i=1}^{2}\left|n_{i}\right|_{\mathbf{X}_{T}^{2}}^{6}\right) .
$$

Thirdly, it was proved in [5, Lemma 2.6], where the notation $B_{2}\left(u_{i}, n_{i}\right)$ was used in place of $u_{i} \cdot \nabla n_{i}$, that there exists a constant $C>0$ such that for all $u_{i} \in \mathrm{V}$ and $n_{i} \in D\left(\hat{\mathrm{A}}^{\frac{3}{2}}\right), i=1,2$, we have

$$
\begin{aligned}
\left|u_{1} \cdot \nabla n_{1}-u_{2} \cdot \nabla n_{2}\right|_{\mathrm{H}^{1}} \leq & C\left(\left|\nabla\left(u_{1}-u_{2}\right)\right|_{L^{2}}\left|n_{1}\right|_{\mathrm{H}^{2}}^{\frac{1}{2}}\left|n_{1}\right|_{\mathrm{H}^{3}}^{\frac{1}{2}}\right. \\
& \left.+\left|n_{1}-n_{2}\right|_{\mathrm{H}^{2}}^{\frac{1}{2}}\left|n_{1}-n_{2}\right|_{\mathrm{H}^{3}}^{\frac{1}{2}}\left|\nabla u_{2}\right|_{L^{2}}\right) .
\end{aligned}
$$

From this inequality we easily infer that there exists a constant $C>0$ such that for all $\left(u_{i}, n_{i}\right) \in \mathbf{X}_{T}^{1} \times \mathbf{X}_{T}^{2}, i=1,2$

$$
\begin{aligned}
\left|u_{1} \cdot \nabla n_{1}-u_{2} \cdot \nabla n_{2}\right|_{L^{2}\left(0, T ; \mathrm{H}^{1}\right)}^{2} \leq & C\left(\left|u_{1}-u_{2}\right|_{C([0, T] ; \mathrm{V})}^{2}\left|n_{1}\right|_{C([0, T] ; D(\hat{\mathrm{A}}))} \int_{0}^{T}\left|n_{1}(s)\right|_{\mathrm{H}^{3}} \mathrm{~d} s\right. \\
& \left.+\left|\nabla u_{2}\right|_{C([0, T] ; \mathrm{V})}^{2}\left|n_{1}-n_{2}\right|_{C([0, T] ; D(\hat{\mathrm{A}}))} \int_{0}^{T}\left|n_{1}(s)-n_{2}(s)\right|_{\mathrm{H}^{3}} \mathrm{~d} s\right)
\end{aligned}
$$




$$
\leq C T^{\frac{1}{2}}\left(\left|u_{1}-u_{2}\right|_{\mathbf{X}_{T}^{1}}^{2}\left|n_{1}\right|_{\mathbf{X}_{T}^{2}}^{2}+\left|u_{2}\right|_{\mathbf{X}_{T}^{1}}^{2}\left|n_{1}-n_{2}\right|_{\mathbf{X}_{T}^{2}}^{2}\right)
$$

Fourthly, we prove that there exists a constant $C>0$ such that for all $n_{i} \in \mathbf{X}_{T}^{2}$, $i=1,2$,

$$
\left.|| \nabla n_{1}\right|^{2} n_{1}-\left.\left|\nabla n_{2}\right|^{2} n_{2}\right|_{L^{2}\left(0, T ; \mathrm{H}^{1}\right)} ^{2} \leq C\left(T \vee T^{\frac{1}{2}}\right)\left|n_{1}-n_{2}\right|_{\mathbf{X}_{T}^{2}}^{2}\left(\left|n_{1}\right|_{\mathbf{X}_{T}^{2}}^{4}+\left|n_{2}\right|_{\mathbf{X}_{T}^{2}}^{4}\right) .
$$

We need the following claim to prove this.

Claim A.1. There exists $C>0$ such that for $a, b \in D\left(\hat{\mathrm{A}}^{\frac{3}{2}}\right)$ and $c \in D(\hat{\mathrm{A}})$ the following holds

$$
|[\nabla a: \nabla b] c|_{\mathrm{H}^{1}}^{2} \leq|a|_{\mathrm{H}^{2}}^{2}|b|_{\mathrm{H}^{2}}^{2}|c|_{\mathrm{H}^{2}}^{2}+|c|_{\mathrm{H}^{2}}^{2}\left[|a|_{\mathrm{H}^{2}}|a|_{\mathrm{H}^{3}}|b|_{\mathrm{H}^{2}}^{2}+|a|_{\mathrm{H}^{2}}^{2}|b|_{\mathrm{H}^{2}}|b|_{\mathrm{H}^{3}}\right]
$$

Proof of Claim A.1. We infer from the Cauchy-Schwarz inequality and the GagliardoNirenberg inequality ([1, Section 9.8, Example C.3]) that there exists a constant $C>0$ such that for all $a, b \in D\left(\hat{\mathrm{A}}^{\frac{3}{2}}\right)$ and $c \in D(\hat{\mathrm{A}})$ we have

$$
\begin{aligned}
& \quad|[\nabla a: \nabla b] c|_{\mathrm{H}^{1}}^{2}=|[\nabla a: \nabla b] c|_{L^{2}}^{2}+\sum_{i=1}^{2}\left|\partial_{i}([\nabla a: \nabla b] c)\right|_{L^{2}}^{2} \\
& \leq C|\nabla a|_{L^{4}}^{2}|\nabla b|_{L^{4}}^{2}|c|_{L^{\infty}}^{2}+C|c|_{L^{\infty}}^{2} \sum_{i=1}^{2}\left(\left|\nabla \partial_{i} a\right|_{L^{4}}^{2}|\nabla b|_{L^{4}}^{2}+|\nabla a|_{L^{4}}^{2}\left|\nabla \partial_{i} b\right|_{L^{4}}^{2}\right) \\
& \quad+C \sum_{i=1}^{2}|\nabla a|_{L^{8}}^{2}|\nabla b|_{L^{8}}^{2}\left|\partial_{i} c\right|_{L^{4}}^{2} \\
& \leq C|a|_{\mathrm{H}^{2}}^{2}|b|_{\mathrm{H}^{2}}^{2}|c|_{\mathrm{H}^{2}}^{2}+C|c|_{\mathrm{H}^{2}}^{2} \sum_{i=1}^{2}\left(\left|\nabla \partial_{i} a\right|_{L^{2}}\left|\nabla \partial_{i} a\right|_{\mathrm{H}^{1}}|b|_{\mathrm{H}^{2}}^{2}\right. \\
& \left.\quad+|\nabla a|_{\mathrm{H}^{2}}^{2}\left|\nabla \partial_{i} b\right|_{L^{2}}\left|\nabla \partial_{i} b\right|_{\mathrm{H}^{1}}\right) \\
& \quad+C \sum_{i=1}^{2}|\nabla a|_{\mathrm{H}^{1}}^{2}|\nabla b|_{\mathrm{H}^{1}}^{2}\left|\partial_{i} c\right|_{L^{4}}^{2} \\
& \leq C|a|_{\mathrm{H}^{2}}^{2}|b|_{\mathrm{H}^{2}}^{2}|c|_{\mathrm{H}^{2}}^{2}+|c|_{\mathrm{H}^{2}}^{2}\left(|a|_{\mathrm{H}^{2}}|a|_{\mathrm{H}^{3}}|b|_{\mathrm{H}^{2}}^{2}+|a|_{\mathrm{H}^{2}}^{2}|b|_{\mathrm{H}^{2}}|b|_{\mathrm{H}^{3}}\right) .
\end{aligned}
$$

Thus, the proof of Claim A.1 is complete.

Let us resume the proof of (A.6). Applying the claim and integrating over $[0, T]$ yields

$$
\begin{aligned}
& \left|\left[\nabla n_{2}: \nabla n_{2}\right]\left(n_{1}-n_{2}\right)\right|_{L^{2}\left(0, T ; \mathrm{H}^{1}\right)}^{2} \leq C T\left|n_{2}\right|_{C([0, T ; D(\hat{\mathrm{A}})])}^{4}\left|n_{1}-n_{2}\right|_{C([0, T ; D(\hat{\mathrm{A}})])}^{2} \\
& \quad+C\left|n_{1}-n_{2}\right|_{C([0, T ; D(\hat{\mathrm{A}})])}^{2}\left(\left|n_{2}\right|_{C([0, T ; D(\hat{\mathrm{A}})])}^{3} \int_{0}^{T}\left|n_{2}(s)\right|_{\mathrm{H}^{3}} \mathrm{~d} s\right)
\end{aligned}
$$




$$
\begin{aligned}
\leq & C T^{\frac{1}{2}}\left|n_{1}-n_{2}\right|_{\mathbf{X}_{T}^{2}}^{2}\left(\left|n_{2}\right|_{\mathbf{X}_{T}^{2}}^{2}\left|n_{2}\right|_{C([0, T ; D(\hat{\mathrm{A}})])}\left(\int_{0}^{T}\left|n_{2}(s)\right|_{\mathrm{H}^{3}}^{2} \mathrm{~d} s\right)^{\frac{1}{2}}\right) \\
& +C T\left|n_{2}\right|_{\mathbf{X}_{T}^{2}}^{4}\left|n_{1}-n_{2}\right|_{\mathbf{X}_{T}^{2}}^{2},
\end{aligned}
$$

for some constant $C>0$ and for all $n_{i} \in \mathbf{X}_{T}^{2}, i=1,2$. The last line of the above chain of inequalities yields that there exists a constant $C>0$ such that for all $n_{i} \in \mathbf{X}_{T}^{2}$, $i=1,2$,

$$
\left|\left[\nabla n_{2}: \nabla n_{2}\right]\left(n_{1}-n_{2}\right)\right|_{L^{2}\left(0, T ; \mathrm{H}^{1}\right)}^{2} \leq C\left(T \vee T^{\frac{1}{2}}\right)\left|n_{2}\right|_{\mathbf{X}_{T}^{2}}^{4}\left|n_{1}-n_{2}\right|_{\mathbf{X}_{T}^{2}}^{2} .
$$

In a similar way, one can show that there exists a constant $C>0$ such that for all $n_{i} \in \mathbf{X}_{T}^{2}, i=1,2$,

$$
\left|\left[\nabla\left(n_{1}-n_{2}\right): \nabla\left(n_{1}+n_{2}\right)\right] n_{1}\right|_{L^{2}\left(0, T ; \mathrm{H}^{1}\right)}^{2} \leq C\left(T \vee T^{\frac{1}{2}}\right)\left|n_{1}-n_{2}\right|_{\mathbf{X}_{T}^{2}}^{2}\left(\left|n_{2}\right|_{\mathbf{X}_{T}^{2}}^{4}+\left|n_{1}\right|_{\mathbf{X}_{T}^{2}}^{4}\right) .
$$

We easily complete the proof of (A.6) by using (A.7) and (A.8) in the following inequality

$$
\begin{aligned}
\left.|| \nabla n_{1}\right|^{2} n_{1}-\left.\left|\nabla n_{2}\right|^{2} n_{2}\right|_{L^{2}\left(0, T ; \mathrm{H}^{1}\right)} ^{2} \leq & 2\left|\left[\nabla\left(n_{1}-n_{2}\right): \nabla\left(n_{1}+n_{2}\right)\right] n_{1}\right|_{L^{2}\left(0, T ; \mathrm{H}^{1}\right)}^{2} \\
+ & +2\left|\left[\nabla n_{2}: \nabla n_{2}\right]\left(n_{2}-n_{1}\right)\right|_{L^{2}\left(0, T ; \mathrm{H}^{1}\right)}^{2} .
\end{aligned}
$$

In order to complete the proof of Lemma 3.9 we need to establish the inequality (3.13). For this purpose, we firstly observe that it is not difficult to prove that there exists a constant $C>0$ such that for all $n_{i}, i=1,2$,

$$
\begin{aligned}
& \left|\left(n_{1}-n_{2}\right) \times g\right|_{L^{2}\left(0, T ; \mathrm{H}^{1}\right)}^{2} \\
& \quad \leq\left|n_{1}-n_{2}\right|_{C([0, T] ; D(\hat{\mathrm{A}}))}^{2}|g|_{L^{2}\left(0, T ; \mathrm{H}^{1}\right)}^{2}+\left|\nabla\left(n_{1}-n_{2}\right)\right|_{C\left([0, T] ; L^{4}\right)}^{2} \int_{0}^{T}|g(s)|_{L^{4}}^{2} \mathrm{~d} s .
\end{aligned}
$$

Hence, there exists a constant $C>0$ such that for all $n_{i} \in \mathbf{X}_{T}^{2}, i=1,2$, we have

$$
\left|\left(n_{1}-n_{2}\right) \times g\right|_{L^{2}\left(0, T ; \mathrm{H}^{1}\right)}^{2} \leq\left|n_{1}-n_{2}\right|_{\mathbf{X}_{T}^{2}}^{2}|g|_{L^{2}\left(0, T ; \mathrm{H}^{1}\right)}^{2},
$$

which is the inequality (3.13). Hence, the proof of Lemma 3.9 is complete.

\section{Appendix B: Weak solution of a modified viscous transport equation}

Let $d \in C\left([0, T] ; \mathrm{H}^{1}\right) \cap L^{2}\left(0, T ; \mathrm{H}^{2}\right)$ and $u \in C([0, T] ; \mathrm{H}) \cap L^{2}(0, T ; \mathrm{V})$. Consider the following problem

$$
\left\{\begin{array}{l}
\partial_{t} z-\Delta z=2|\nabla d|^{2} z-2\left(\phi^{\prime}(d) \cdot d\right) z-u \cdot \nabla z \\
\left.\frac{\partial z}{\partial v}\right|_{\partial \Omega}=0 \\
z(0)=z_{0} .
\end{array}\right.
$$

We introduce the definition of weak solution of problem B.1. 
Definition B.1. Let $z_{0} \in L^{2}$. A function $z:[0, T] \rightarrow L^{2}$ is a weak solution to (B.1) iff

(1) $z \in C\left([0, T] ; L^{2}\right) \cap L^{2}\left(0, T ; H^{1}\right)$;

(2) for all $t \in[0, T]$ and $\varphi \in \mathrm{H}^{1}$

$$
\begin{aligned}
& \left(z(t)-z_{0}, \varphi\right)+\int_{0}^{t}(\nabla z(s), \nabla \varphi) \mathrm{d} s \\
& \quad=\int_{0}^{t}\left(2|\nabla d(s)|^{2} z(s)-2\left(\phi^{\prime}(d(s)) \cdot d(s)\right) z(s)-u(s) \cdot \nabla z(s), \varphi\right) \mathrm{d} s
\end{aligned}
$$

Remark B.2. Let $d \in C\left([0, T] ; \mathrm{H}^{1}\right) \cap L^{2}\left(0, T ; \mathrm{H}^{2}\right), u \in C([0, T] ; \mathrm{H}) \cap L^{2}(0, T ; \mathrm{V})$ and $z \in C\left([0, T] ; L^{2}\right) \cap L^{2}\left(0, T ; H^{1}\right)$ be a weak solution to (3.23). Then, by using the Hölder inequality and Sobolev embeddings such as $\mathrm{H}^{1} \hookrightarrow L^{4}$ and $\mathrm{H}^{2} \hookrightarrow \infty$ we can easily shows that

$$
\begin{aligned}
\left|\partial_{t} z\right|_{\left(\mathrm{H}^{1}\right)^{*}} & =\sup _{\varphi \in \mathrm{H}^{1}:|\varphi|_{\mathrm{H}^{1}}=1}\left|\left\langle\partial_{t} z, \varphi\right\rangle\right| \\
& \leq|\nabla z|_{L^{2}}+|\nabla d|_{L^{4}}^{2}|z|_{L^{4}}+|u|_{L^{4}}|z|_{L^{4}}+\left(1+|d|_{\mathrm{H}^{2}}\right)|d|_{L^{4}}|z|_{L^{2}} .
\end{aligned}
$$

Hence, since $u, \nabla d, z \in C\left([0, T] ; L^{2}\right) \cap L^{2}\left(0, T ; \mathrm{H}^{1}\right) \subset L^{4}\left(0, T ; L^{4}\right)$, we can show by using the Hölder inequality and (B.3) that

$$
\left|\partial_{t} z\right|_{L^{2}\left(0, T ;\left(\mathrm{H}^{1}\right)^{*}\right)} \leq c
$$

for a universal constant which depends only on $\Omega$ and $T$.

The following result gives the existence and uniqueness of problem (B.1).

Proposition B.3. Let $d \in C\left([0, T] ; \mathrm{H}^{1}\right) \cap L^{2}\left(0, T ; \mathrm{H}^{2}\right), u \in C([0, T] ; \mathrm{H}) \cap$ $L^{2}(0, T ; \mathrm{V})$ and $z_{0} \in L^{2}$. Let also $\phi: \mathbb{R}^{3} \rightarrow \mathbb{R}^{+}$be of class $C^{1}$ such that

$$
\left|\phi^{\prime}(d)\right|_{\mathbb{R}^{3}} \leq c(1+|d|) .
$$

Then problem (B.1) has a unique weak solution z. Moreover, there exists a constant $c>0$ such that

$$
\sup _{0 \leq t \leq T}|z(t)|_{L^{2}}^{2}+\int_{0}^{T}|\nabla z(t)|^{2} \mathrm{~d} t \leq|z(0)|_{L^{2}}^{2} e^{c \int_{0}^{T}\left[|\nabla d|_{L^{4}}^{4}+\left(1+|d|_{\mathrm{H}^{2}}^{2}\right)\right] \mathrm{d} t}
$$

Proof. Throughout this proof $c>0$ will denote an universal constant which depends only on $\Omega$ and may change from one term to the other. For the sake of simplicity we will omit the dependence on the space variable inside any integral over $\Omega$.

Let $d \in C\left([0, T] ; \mathrm{H}^{1}\right) \cap L^{2}\left(0, T ; \mathrm{H}^{2}\right), u \in C([0, T] ; \mathrm{H}) \cap L^{2}(0, T ; \mathrm{V})$ and $z_{0} \in L^{2}$.

Since the problem (B.1) is linear, the proofs of the existence, which can be done via Galerkin and compactness methods, and the uniqueness are easy and omitted. So 
we only prove (B.3). For this purpose, $z \in C\left([0, T] ; L^{2}\right) \cap L^{2}\left(0, T ; \mathrm{H}^{1}\right)$ be a weak solution to (3.23). We firstly observe that since $\mathrm{H}^{2} \subset L^{\infty}$

$$
\begin{aligned}
\int_{\Omega}\left(\phi^{\prime}(d) \cdot d\right)|z|^{2} \mathrm{~d} x & \leq c|d|_{L^{\infty}}\left(1+|d|_{L^{\infty}}\right) \int_{\Omega}|z|^{2} \mathrm{~d} x \\
& \leq c\left(1+|d|_{\mathrm{H}^{2}}^{2}\right)|z|_{L^{2}}^{2} .
\end{aligned}
$$

Also, since $u \in \mathrm{H}_{0}^{1}$ and $\operatorname{div} u=0$ we can prove that

$$
\langle u \cdot \nabla z, z\rangle=\frac{1}{2} \int_{\Omega} u \cdot \nabla|z|^{2} \mathrm{~d} x=0 .
$$

Hence, by the Gagliardo-Nirenberg inequality ([1, Section 9.8, Example C.3]) and the Lions-Magenes lemma ([37, Lemma III.1.2]), which is applicable because of Definition B.1(1) and (B.2), we have the following inequalities

$$
\begin{aligned}
\left\langle\partial_{t} z, z\right\rangle & =\frac{1}{2} \frac{\mathrm{d}}{\mathrm{d} t}|z|_{L^{2}}^{2} \\
& =-\frac{1}{2}|\nabla z|_{L^{2}}^{2}+2 \int_{\Omega}|\nabla d|^{2}|z|^{2} \mathrm{~d} x-2 \int_{\Omega}\left(\phi^{\prime}(d) \cdot d\right)|z|^{2} \mathrm{~d} x \\
& \leq-\frac{1}{2}|\nabla z|_{L^{2}}^{2}+\int_{\Omega}|\nabla d|^{2}|z|^{2} \mathrm{~d} x+C\left(1+|d|_{\mathrm{H}^{2}}^{2}\right)|z|_{L^{2}}^{2} \\
& \leq-\frac{1}{2}|\nabla z|_{L^{2}}^{2}+c|\nabla d|_{L^{4}}^{2}|z|_{L^{4}}^{2}+c\left(1+|d|_{\mathrm{H}^{2}}^{2}\right)|z|_{L^{2}}^{2} \\
& \leq-\frac{1}{2}|\nabla z|_{L^{2}}^{2}+c|\nabla d|_{L^{4}}^{2}|z|_{L^{2}}|\nabla z|_{L^{2}}+|\nabla d|_{L^{4}}^{2}|z|_{L^{2}}^{2}+c\left(1+|d|_{\mathrm{H}^{2}}^{2}\right)|z|_{L^{2}}^{2} \\
& \leq-\frac{1}{2}|\nabla z|_{L^{2}}^{2}+\frac{1}{4}|\nabla z|_{L^{2}}^{2}+c|\nabla d|_{L^{4}}^{4}|z|_{L^{2}}^{2}+c\left(1+|d|_{\mathrm{H}^{2}}^{2}\right)|z|_{L^{2}}^{2} .
\end{aligned}
$$

Thus,

$$
\frac{1}{2} \frac{\mathrm{d}}{\mathrm{d} t}|z|_{L^{2}}^{2}+\frac{1}{4}|\nabla z|_{L^{2}}^{2} \leq c|\nabla d|_{L^{4}}^{4}|z|_{L^{2}}^{2}+c\left(1+|d|_{\mathrm{H}^{2}}^{2}\right)|z|_{L^{2}}^{2}
$$

By applying the Gronwall lemma we infer (B.3). This also completes the proof of Proposition B.3.

\section{Appendix C: Proof of Claim 4.10}

In this section we will the estimates (4.16)-(4.18) in Claim 4.10. Throughout this section $C>0$ will denote an universal constant which may change from one term to the other.

Proof of inequality (4.16). Let us choose and fix $v \in D(\mathrm{~A})$ and $n \in D\left(\hat{\mathrm{A}}^{\frac{3}{2}}\right)$.

Since $D\left(\hat{\mathrm{A}}^{\frac{3}{2}}\right) \subset \mathrm{H}^{3}$ and $\mathrm{H}^{2}$ is an algebra, it is not difficult to show that $\operatorname{div}(\nabla n \odot$ $\nabla n) \in L^{2}$. Thus, using the fact $\Pi: L^{2} \rightarrow \mathrm{H}$ is bounded, the Hölder inequality and Gagliardo-Nirenberg inequality ([1, Section 9.8, Example C.3]) we infer that

$$
|\langle\mathrm{A} v, \Pi[\operatorname{div}(\nabla n \odot \nabla n)]\rangle|=\left|\left\langle\mathrm{A} v, \Pi[\nabla n)^{\mathrm{T}} \Delta n\right]\right\rangle \mid
$$




$$
\begin{aligned}
& \leq C|\mathrm{~A} v|_{L^{2}}|\nabla n|_{L^{4}}|\Delta n|_{L^{4}} \\
& \leq C|\mathrm{~A} v|_{L^{2}}|\nabla n|_{L^{4}}|\Delta n|_{L^{2}}^{\frac{1}{2}}|\nabla \Delta n|_{L^{2}}^{\frac{1}{2}} .
\end{aligned}
$$

Now, applying the Young inequality twice implies

$$
\left|\left\langle\mathrm{A} v, \Pi_{L}[\operatorname{div}(\nabla n \odot \nabla n)]\right\rangle\right| \leq \frac{1}{2}\left(|\mathrm{~A} v|_{L^{2}}^{2}+|\nabla \Delta n|_{L^{2}}^{2}\right)+|\nabla n|_{L^{4}}^{4}|\Delta n|_{L^{2}}^{2} .
$$

This complete the proof of the inequality (4.16).

Proof of the inequality (4.17). Let $v \in D(\mathrm{~A})$ and $n \in D\left(\hat{\mathrm{A}}^{\frac{3}{2}}\right)$ be fixed.

Because $D(\mathrm{~A}) \subset \mathrm{H}^{2}, D\left(\hat{\mathrm{A}}^{\frac{3}{2}}\right) \subset \mathrm{H}^{3}$ and $\mathrm{H}^{\theta}, \theta>1$, is an algebra, it is not difficult to show that $v \cdot \nabla n \in \mathrm{H}^{2} \subset \mathrm{H}^{1}$. Hence, by using the Cauchy-Schwarz and Young inequalities we infer

$$
\begin{aligned}
\left(\mathrm{H}^{1}\right)^{*}\left\langle\mathrm{~A}^{2} n, v \cdot \nabla n\right\rangle_{\mathrm{H}^{1}}= & \langle\nabla \Delta n, \nabla(v \cdot \nabla n)\rangle \\
& \leq \frac{1}{24}|\nabla \Delta n|_{L^{2}}^{2}+C|\nabla(v \cdot \nabla n)|_{L^{2}}^{2} .
\end{aligned}
$$

Using the Hölder inequality in the last line we infer that

$$
\begin{aligned}
\left(\mathrm{H}^{1}\right)^{*}\left\langle\mathrm{~A}^{2} n, v \cdot \nabla n\right\rangle_{\mathrm{H}^{1}} & \leq \frac{1}{24}|\nabla \Delta n|_{L^{2}}^{2}+C|(\nabla v)(\nabla n)|_{L^{2}}^{2}+C|v \cdot \nabla(\nabla n)|_{L^{2}}^{2} \\
& \leq \frac{1}{24}|\nabla \Delta n|_{L^{2}}^{2}+C|(\nabla v)|_{L^{4}}^{2}|(\nabla n)|_{L^{4}}^{2}+C|v|_{L^{4}}^{2}\left|\nabla^{2} n\right|_{L^{4}}^{2}
\end{aligned}
$$

Now, by using [33, Theorem 3.4] to estimate $\left|\nabla^{2} n\right|_{L^{4}}$ by $|\Delta n|_{L^{4}}$, then by applying the Gagliardo-Nirenberg ([1, Section 9.8, Example C.3]), the Young inequalities we infer that

$$
\begin{aligned}
& \left(\mathrm{H}^{1}\right)^{*}\left\langle\mathrm{~A}^{2} n, v \cdot \nabla n\right\rangle_{\mathrm{H}^{1}} \leq \frac{1}{24}|\nabla \Delta n|_{L^{2}}^{2}+C|\nabla v|_{L^{2}}|\mathrm{~A} v|_{L^{2}}|(\nabla n)|_{L^{4}}^{2}+C|v|_{L^{4}}^{2}|\Delta n|_{L^{4}}^{2} \\
& \quad \leq \frac{1}{24}|\nabla \Delta n|_{L^{2}}^{2}+\frac{1}{12}|\mathrm{~A} v|_{L^{2}}^{2}+C|\nabla v|_{L^{2}}^{2}|(\nabla n)|_{L^{4}}^{4}+C|v|_{L^{4}}^{2}\left[|\Delta n|_{L^{2}}|\nabla \Delta n|_{L^{2}}+|\Delta n|_{L^{2}}^{2}\right] \\
& \quad \leq \frac{1}{12}|\nabla \Delta n|_{L^{2}}^{2}+\frac{1}{12}|\mathrm{~A} v|_{L^{2}}^{2}+C|\nabla v|_{L^{2}}^{2}|(\nabla n)|_{L^{4}}^{2}+C\left[|v|_{L^{4}}^{2}+|v|_{L^{4}}^{4}\right]|\Delta n|_{L^{2}} .
\end{aligned}
$$

This completes the proof of the inequality (4.17).

Proof of the inequality (4.18). Let $v \in D(\mathrm{~A})$ and $n \in D\left(\hat{\mathrm{A}}^{\frac{3}{2}}\right)$. As in the proof of (4.16), we can show that $|\nabla n|^{2} n \in \mathrm{H}^{1}$. Hence, using the Cauchy-Schwarz, the Young and the Gagliardo-Nirenberg inequalities (see [1, Section 9.8, Example C.3]) we infer that

$$
\begin{aligned}
\left(\mathrm{H}^{1}\right)^{*}\left\langle\hat{\mathrm{A}}^{2} n,|\nabla n|^{2} n\right\rangle_{\mathrm{H}^{1}} & =\left\langle\nabla \Delta n, \nabla\left(|\nabla n|^{2} n\right)\right\rangle \\
& \leq \frac{1}{24}|\nabla \Delta n|_{L^{2}}^{2}+C\left|\nabla\left(|\nabla n|^{2} n\right)\right|_{L^{2}}^{2} \\
& \leq \frac{1}{24}|\nabla \Delta n|_{L^{2}}^{2}+C\left|\nabla\left(|\nabla n|^{2} n\right)\right|_{L^{2}}^{2}
\end{aligned}
$$




$$
\begin{aligned}
& \leq \frac{1}{24}|\nabla \Delta n|_{L^{2}}^{2}+C\left|(\nabla n)\left(\nabla^{2} n\right)\right|_{L^{2}}^{2}+\left.\left.C|| \nabla n\right|^{2} \nabla n\right|_{L^{2}} ^{2} \\
& \leq \frac{1}{24}|\nabla \Delta n|_{L^{2}}^{2}+C|(\nabla n)|_{L^{4}}^{2}\left|\left(\nabla^{2} n\right)\right|_{L^{4}}^{2}+C|\nabla n|_{L^{6}}^{6} \\
& \leq \frac{1}{24}|\nabla \Delta n|_{L^{2}}^{2}+C|(\nabla n)|_{L^{4}}^{2}\left|\left(\nabla^{2} n\right)\right|_{L^{4}}^{2}+C|\nabla n|_{L^{4}}^{4}\left(|\nabla n|_{L^{2}}^{2}+\left|\nabla^{2} n\right|_{L^{2}}^{2}\right) .
\end{aligned}
$$

Proceeding as in the proof of (C.1), we show that

$$
\begin{aligned}
\left(\mathrm{H}^{1}\right)^{*}\left\langle\hat{\mathrm{A}}^{2} n,|\nabla n|^{2} n\right\rangle_{\mathrm{H}^{1}} \leq & \frac{1}{12}|\nabla \Delta n|_{L^{2}}^{2}+C\left[|(\nabla n)|_{L^{4}}^{4}+|(\nabla n)|_{L^{4}}^{2}\right]|(\Delta n)|_{L^{2}}^{2} \\
& +C|\nabla n|_{L^{4}}^{4}\left(|\nabla n|_{L^{2}}^{2}+|\Delta n|_{L^{2}}^{2}\right) .
\end{aligned}
$$

This completes the proof of (4.18).

\section{Appendix D: A weak continuity of banach space valued functions}

In this section we will state and prove of continuity theorem for Banach-valued map similar to [35]. This theorem was initially proven in the work in progress [6], but for the sake of completeness we repeat the proof here.

Theorem D.1. Let $X$ and $Y$ be two Banach spaces such that $X$ is reflexive, $X \subset Y$ and the canonical injection $i: X \rightarrow Y$ is dense and continuous. Let $T>0$ be fixed and $u \in L^{\infty}([0, T) ; X)$. Let also $b \in Y$ and $v:[0, T] \rightarrow Y$, defined by

$$
v(t)=\left\{\begin{array}{l}
i(u(t)) \text { if } t \in[0, T), \\
b \text { if } t=T,
\end{array}\right.
$$

be weakly continuous.

Then, $b \in X$ and the map $\tilde{u}:[0, T] \rightarrow X$ defined by

$$
\tilde{u}(t)=\left\{\begin{array}{l}
u(t) \text { if } t \in[0, T), \\
b \text { if } t=T,
\end{array}\right.
$$

is weakly continuous.

Proof. Let $X, Y, b \in Y, T>0, u \in L^{\infty}([0, T) ; X), v:[0, T] \rightarrow Y$ be as in the statement of the theorem. Let also $\left(t_{n}\right)_{n \in \mathbb{N}} \subset[0, T)$ be a sequence such that $t_{n} \nearrow T$.

Let us prove the first part of the theorem, i.e., that $b \in X$. For this aim we observe that by assumption there exists $M>0$ such that

$$
|u(t)|_{X} \leq M \text { for all } t \in[0, T) .
$$

Hence, by the Banach-Alaoglu theorem we can extract from $\left(t_{n}\right)_{n \in \mathbb{N}}$ a subsequence, which is still denoted by $\left(t_{n}\right)_{n \in \mathbb{N}}$, such that $t_{n} \nearrow T$ and

$$
u\left(t_{n}\right) \rightarrow x \text { weakly in } X .
$$


Since, by assumption, $X \subset Y$ we infer that $v(t)=i(u(t))=u(t)$ for all $t \in[0, T]$. Hence, by the weak continuity of $v$ we infer that

$$
v\left(t_{n}\right)=u\left(t_{n}\right) \rightarrow b \text { weakly in } Y .
$$

By the uniqueness of weak limit we infer that $x=b \in X$.

It remains to prove the second part of the theorem, i.e., we shall show that the map $\tilde{u}:[0, T] \rightarrow X$ defined in (D.1) is weakly continuous. For this purpose, we will closely follow the proof of [37, Lemma III.1.4 ]. We will divide the task into two cases.

Case 1 Let $t, t_{0} \in[0, T)$. Let $X^{*}$ and $Y^{*}$ be the dual spaces of $X$ and $Y$, respectively. Recall that since the embedding $X \subset Y$ is dense and continuous, the embedding $Y^{*} \subset X^{*}$ is dense and continuous, too. Let us choose and fix $\varepsilon>0$ and $\eta \in X^{*}$. Then, there exists $\eta_{\varepsilon} \in Y^{*}$ such that

$$
\left|\eta-\eta_{\varepsilon}\right|_{X^{*}}<\varepsilon \text {. }
$$

Thus, using the boundedness of $u=\left.\tilde{u}\right|_{[0, T)}$ we infer that

$$
\begin{aligned}
\left.\right|_{X}\left\langle\tilde{u}(t)-\tilde{u}\left(t_{0}\right), \eta\right\rangle_{X^{*}} \mid & \leq\left.\right|_{X}\left\langle\tilde{u}(t)-\tilde{u}\left(t_{0}\right), \eta-\eta_{\varepsilon}\right\rangle_{X^{*}}|+|_{Y}\left\langle\tilde{u}(t)-\tilde{u}\left(t_{0}\right), \eta\right\rangle_{Y^{*}} \mid \\
& \leq 2 M\left|\eta-\eta_{\varepsilon}\right|_{X^{*}}+\left.\right|_{Y}\left\langle\tilde{u}(t)-\tilde{u}\left(t_{0}\right), \eta\right\rangle_{Y^{*}} \mid .
\end{aligned}
$$

By the weak continuity of $\left.v\right|_{[0, T)}=u=\tilde{u}$ we have

$$
\left.\lim _{t \rightarrow t_{0}}\right|_{X}\left\langle\tilde{u}(t)-\tilde{u}\left(t_{0}\right), \eta\right\rangle_{X^{*}}|\leq 2 M| \eta-\left.\eta_{\varepsilon}\right|_{X^{*}}+\left.\lim _{t \rightarrow t_{0}}\right|_{Y}\left\langle v(t)-v\left(t_{0}\right), \eta\right\rangle_{Y^{*}} \mid \leq 2 M \varepsilon .
$$

Since $\varepsilon>0$ is arbitrary, we infer that

$$
\left.\lim _{t \rightarrow t_{0}}\right|_{X}\left\langle\tilde{u}(t)-\tilde{u}\left(t_{0}\right), \eta\right\rangle_{X^{*}} \mid=0 .
$$

Hence, $\left.\tilde{u}\right|_{[0, T)}$ is weakly continuous.

Case 2 We will prove that $\tilde{u}$ is weakly continuous at $t=T$. Toward this aims let $\eta \in X^{*}, \varepsilon>0$ be fixed. From the proof of the first part of the theorem we infer that there exists $\delta>0$ such that for $t>0$ with $0<T-t<\delta$

$$
{ }_{X}\langle u(t)-b, \eta\rangle_{X^{*}}\left|={ }_{X}\langle u(t)-\tilde{u}(T), \eta\rangle_{X^{*}}\right|<\varepsilon .
$$

But for $t \in(0, T)$ we have $u(t)=\tilde{u}(t)$, hence for $t>0$ with $0<T-t<\delta$

$$
{ }_{X}\langle\tilde{u}(t)-\tilde{u}(T), \eta\rangle_{X^{*}}|\leq|_{X}\langle\tilde{u}(t)-u(t), \eta\rangle_{X^{*}}|+|_{X}\langle u(t)-\tilde{u}(T), \eta\rangle_{X^{*}} \mid<\varepsilon .
$$

Thus, $\tilde{u}$ is weakly continuous at $t=T$. This completes the proof of Case 2 , the second part of the theorem and hence the whole theorem. 


\section{REFERENCES}

[1] H. Brezis. Functional analysis, Sobolev spaces and partial differential equations. Universitext. Springer, New York, 2011.

[2] Z. Brzeźniak, S. Cerrai and M. Freidlin, Quasipotential and exit time for 2D stochastic NavierStokes equations driven by space time white noise, Probab. Theory Relat. Fields 162, pp 739-793, 2015.

[3] Z. Brzeźniak, B. Goldys and T. Jegaraj, Terence . Weak solutions of a stochastic Landau-LifshitzGilbert equation. Appl. Math. Res. Express., 2013 no. 1, 1-33, 2013.

[4] Z. Brzeźniak, E. Hausenblas and P. Razafimandimby. Some results on the penalised nematic liquid crystals driven by multiplicative noise: weak solution and maximum principle. Stoch. Partial Differ. Equ. Anal. Comput. 7 , no. 3, 417-475, 2019.

[5] Z. Brzeźniak, E. Hausenblas and P. Razafimandimby. Stochastic nonparabolic dissipative systems modeling the flow of liquid crystals: strong solution. In: RIMS Symposium on Mathematical Analysis of Incompressible Flow, February 2013. RIMS Kôkyûroku 1875 , pp. 41-73, 2014.

[6] Z. Brzeźniak, U. Manna and A. A. Panda. Stochastic Landau-Lifshitz-Gilbert equations via WongZakai approximation. In preparation.

[7] Y. Chen, S. Kim and Y. Yu. Freedericksz transition in nematic liquid crystal flows in dimension two. SIAM J. Math. Anal. 50, no. 5, pp 4838-4860, 2018.

[8] B. Climent-Ezquerra, F. Guillén-González. A review of mathematical analysis of nematic and smectic-A liquid crystal models. European J. Appl. Math. 25, no 1, pp 133-153, 2014.

[9] P. G. de Gennes and J. Prost. The Physics of Liquid Crystals. Clarendon Press, Oxford

[10] J. L. Ericksen. Conservation laws for liquid crystals. Trans. Soc. Rheology, 5, pp 23-34, 1961.

[11] E. Feireisl, M. Petcu and D. Pražák. Relative energy approach to a diffuse interface model of a compressible two-phase flow. Math. Methods Appl. Sci. 42, no. 5, 1465-1479, 2019.

[12] E. Feireisl, M. Frémond, E. Rocca, Elisabetta and G. Schimperna. A new approach to non-isothermal models for nematic liquid crystals. Arch. Ration. Mech. Anal. 205, no. 2, 651-672, 2012.

[13] E. Feireisl, E. Rocca, Elisabetta and G. Schimperna. . On a non-isothermal model for nematic liquid crystals. Nonlinearity 24, no. 1, 243-257, 2011.

[14] M. Hieber and J. W. Prüss Dynamics of the Ericksen-Leslie equations with general Leslie stress I: the incompressible isotropic case, Math. Ann., 369, pp 977-996, 2017.

[15] M. Hieber and J. W. Prüss Modeling and analysis of the Ericksen-Leslie equations for nematic liquid crystal flows. In: Giga, Y., Novotny, A. (eds.) Handbook of Mathematical Analysis in Mechanics of Viscous Fluids, pp. 1075-1134. Springer, Berlin, 2018.

[16] M. Hieber, M. Nesensohn, J. Prüss and K. Schade. Dynamics of nematic liquid crystal flows: The quasilinear approach, Ann. Inst. H. Poincaré Anal. Non Linéaire, 33, pp 397-408, 2016.

[17] M.-C. Hong. Global existence of solutions of the simplified Ericksen-Leslie system in dimension two. Calc. Var. Partial Differential Equations. 40, pp 15-36, 2011.

[18] M.-C. Hong and Z. Xin. Global existence of solutions of the liquid crystal flow for the Oseen-Frank model in $\mathbb{R}^{2}$. Adv. Math. 231, no 3-4, pp 1364-1400, 2012. . Adv. Math. 231, no 3-4, pp 1364-1400, 2012.

[19] M.-C. Hong, J. Li and Z. Xin. Blow-up criteria of strong solutions to the Ericksen-Leslie system in $\mathbb{R}^{3}$. Comm. Partial Differential Equations 39, no 7, pp 1284-1328, 2014.

[20] M.-C.. Hong and Y. Mei . Well-posedness of the Ericksen-Leslie system with the Oseen-Frank energy in $L_{\text {uloc }}^{3}\left(\mathbb{R}^{3}\right)$. Calc. Var. Partial Differential Equations 58, no. 1, Art. 3, 38 pp, 2019.

[21] J. Huang, F. Lin, Fanghua and C. Wang. Regularity and existence of global solutions to the EricksenLeslie system in $\mathbb{R}^{2}$. Comm. Math. Phys. 331, no 2, pp 805-850, 2014.

[22] T. Huang, F. Lin, C. Liu and C. Wang. Finite time singularity of the nematic liquid crystal flow in dimension three. Arch. Ration. Mech. Anal. 221, no 3, pp 1223-1254, 2016.

[23] F. M. Leslie. Some constitutive equations for liquid crystals. Arch. Rational Mech. Anal. 28, no 4, pp 265-283, 1968.

[24] J. Li, E. S. Titi and Z. Xin. On the uniqueness of weak solutions to the Ericksen-Leslie liquid crystal model in $\mathbb{R}^{2}$. Math. Models Methods Appl. Sci. 26, no. 4, pp 803-822, 2016.

[25] F. Lin and C. Wang. Global existence of weak solutions of the nematic liquid crystal flow in dimension three. Comm. Pure Appl. Math. 69, no 8, pp 1532-1571, 2016. 
[26] F. Lin, J. Lin and C. Wang. Liquid crystal flows in two dimensions. Arch. Rational Mech. Anal. 197, pp 297-336, 2010.

[27] F. H. Lin and C. Liu. Nonparabolic dissipative systems modeling the flow of Liquid Crystals. Communications on Pure and Applied Mathematics, Vol. XLVIII, pp 501-537, 1995.

[28] F.-H. Lin and C. Liu. Existence of solutions for the Ericksen-Leslie System. Arch. Rational Mech. Anal. 154, pp 135-156, 2000.

[29] F. Lin and C. Wang. Recent developments of analysis for hydrodynamic flow of nematic liquid crystals, Philos. Trans. R. Soc. Lond. Ser. A Math. Phys. Eng. Sci. 372, 20130361, 18 pp, 2014.

[30] F. Lin and C. Wang. On the uniqueness of heat flow of harmonic maps and hydrodynamic flow of nematic liquid crystals. Chinese Annals of Mathematics, Series B. 31B, no (6), pp 921-938, 2010.

[31] C. Liu and H. Sun. On energetic variational approaches in modeling the nematic liquid crystal flows. Discret Cont. Dyn. Syst. 23, pp 455-475, 2009.

[32] R. Schoen and K. Uhlenbeck. Boundary regularity and the Dirichlet problem for harmonic maps. J. Differential Geom. 18, no. 2, pp 253-268, 1983.

[33] C. G. Simader, The weak Dirichlet and Neumann problem for the Laplacian in Lq for bounded and exterior domains. Applications, in Nonlinear Analysis, Function Spaces and Applications, Vol. 4, Teubner-Texte Mathematik (Teubner), pp. 180-223, 1990.

[34] I. W. Stewart, The Static and Dynamic Continuum Theory of Liquid Crystals: A Mathematical Introduction, CRC Press, Boca Raton, FL, 2004.

[35] W.A. Strauss, On continuity of functions with values in various Banach spaces, Pacific J. Math. 19, pp 543-551, 1966.

[36] M. Struwe. On the evolution of harmonic maps of Riemannian surfaces. Commun. Math. Helv. 60, pp 558-581, 1985.

[37] R. Temam, Navier-Stokes equations. Theory and numerical analysis, Reprint of the 1984 edition. AMS Chelsea Publishing, Providence, RI, 2001. xiv+408 pp.

[38] R. Temam. Infinite-dimensional dynamical systems in mechanics and physics. Second edition. Applied Mathematical Sciences, 68. Springer-Verlag, New York, 1997.

[39] R. Temam, Navier-Stokes equations and nonlinear functional analysis. Second edition. CBMSNSF Regional Conference Series in Applied Mathematics, 66. Society for Industrial and Applied Mathematics (SIAM), Philadelphia, PA, 1995.

[40] M. Wang and W. Wang. Global existence of weak solution for the 2-D Ericksen-Leslie system. Calc. Var. Partial Differential Equations, 51, no 3-4, pp 915-962, 2014.

[41] W. Wang, P. Zhang and Z. Zhang. Well-posedness of the Ericksen-Leslie system. Arch. Ration. Mech. Anal. 210, no 3, pp 837-855, 2013.

[42] M. Wang, W. Wang and Z. Zhang. On the uniqueness of weak solution for the 2-D Ericksen-Leslie system. Discrete Contin. Dyn. Syst. Ser. B 21, no 3, pp 919-941, 2016.

[43] W. Wang, P. Zhang and Z. Zhang. The small Deborah number limit of the Doi-Onsager equation to the Ericksen-Leslie equation. Comm. Pure Appl. Math. 68, no 8, pp 1326-1398, 2015. 
Zdzislaw Brzeźniak and Paul André Razafimandimby Department of Mathematics

University of York

Heslington, York YO10 5DD

$U K$

E-mail: zdzislaw.brzezniak@york.ac.uk

Paul André Razafimandimby

E-mail: paul.razafimandimby@york.ac.uk

\section{Gabriel Deugoué}

Department of Mathematics and Computer Science University of Dschang

Dschang

Cameroon

E-mail:agdeugoue@yahoo.fr

Accepted: 22 April 2021 